BULLETIN (New Series) OF THE

AMERICAN MATHEMATICAL SOCIETY

Volume 48, Number 2, April 2011, Pages 155-209

S 0273-0979(2011)01326-X

Article electronically published on January 18, 2011

\title{
HOW CAN WE CONSTRUCT ABELIAN GALOIS EXTENSIONS OF BASIC NUMBER FIELDS?
}

\author{
BARRY MAZUR
}

\begin{abstract}
Irregular primes - 37 being the first such prime-have played a great role in number theory. This article discusses Ken Ribet's constructionfor all irregular primes $p$ - of specific abelian, unramified, degree $p$ extensions of the number fields $\mathbf{Q}\left(e^{2 \pi i / p}\right)$. These extensions with explicit information about their Galois groups (they are Galois over $\mathbf{Q}$ ) were predicted to exist ever since the work of Herbrand in the 1930s. Ribet's method involves a tour through the theory of modular forms; it demonstrates the usefulness of congruences between cuspforms and Eisenstein series, a fact that has inspired, and continues to inspire, much work in number theory.
\end{abstract}

\section{INTRODUCTION}

Recall the almost tautological - but very useful - way we have of dealing with an irreducible polynomial over a field. For example, in the case of $X^{5}-X+1$, irreducible over the rational field $\mathbf{Q}$, we happily adjoin a root to our base field by merely forming $L:=$ the quotient of the polynomial ring $\mathbf{Q}[X]$ modulo the ideal generated by $X^{5}-X+1$. Then $L$ is a field (of algebraic numbers) and the image of $X$ is indeed a root of $X^{5}-X+1$ in $L$.

This method is serviceable, as far as it goes 1 but sometimes the field extensions that we are interested in, the field extensions that we expect - thanks to some heuristic or other-should exist, would not be readily constructible this way. Moreover, once constructed, they might not be understandable, nor treatable, this way, i.e., in terms of polynomials whose roots generate them, even if those polynomials were readily available. Such is the case, for the most part, for the abelian extensions alluded to in the title above.

This article is based on a talk I gave entitled Construction of Abelian Extensions Following Ken Ribet at the 60th Birthday Conference for Ken Ribet (held at the University of California at Berkeley and MSRI June 28-July 2, 2008)2 My mission was to focus on Ribet's method of construction of abelian Galois extensions of

Received by the editors September 20, 2009, and, in revised form, January 29, 2010.

2010 Mathematics Subject Classification. Primary 11R04, 18-XX, 20-XX, 23-XX.

${ }^{1}$ On occasion, it works quite nicely, as in the construction of cyclotomic fields in Section 2 below.

${ }^{2}$ I want to thank Joël Bellaïche, Gaëtan Chenevier, Chandan Dalawat, Ralph Greenberg, Michael Harris, William Stein, Eric Urban, and the referee who have helped me with comments and corrections regarding early drafts of this article. My thanks go, as well, to Sasha Makarova for help with the figures.

(C)2011 American Mathematical Society Reverts to public domain 28 years from publication 


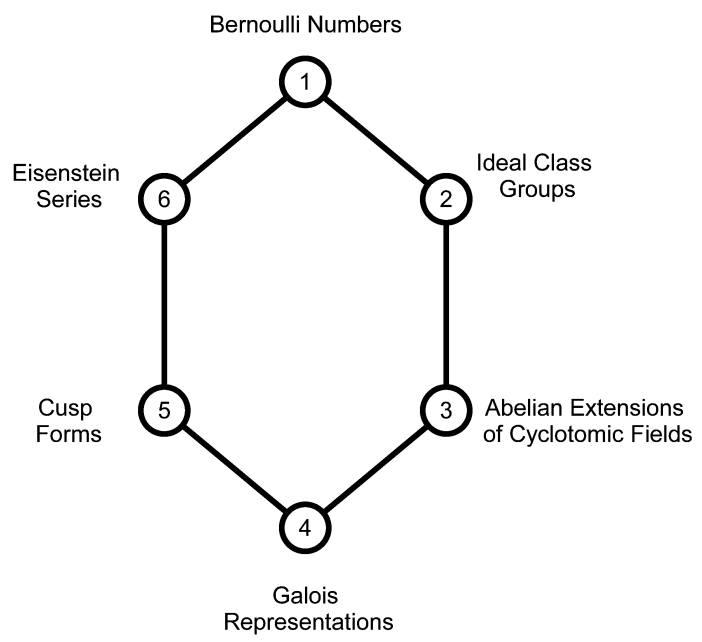

FIGURE 1

cyclotomic fields - one of Ken's great early achievements - and to hint at the vast influence this work has had in the later development of our subject 3

The central topic of this article is the theorem referred to nowadays simply as the Herbrand-Ribet Theorem.

The first thing to know about this theorem is that Ken's 11-page paper 49 . from which much of this mathematics stems is as worth reading today as it was over three decades ago, and is eminently readable. For this reason, one aim of this article is not to give a proof of the theorem (I do not) but rather to try to explain why the ideas behind the theorem have played such an inspirational role in the subject, and why they will continue to do so.

The second thing to know is that the Herbrand-Ribet Theorem for a prime number $p$ concerns six slightly different facets of number theory, specifically as they are related to $p$, and weaves them together in a striking way. This is illustrated in Figure 1 .

This article is divided into four parts and two appendices.

Part I is a general introduction to the hexagon in Figure 1 and a discussion of how its vertices are linked together. We will do this by circumnavigating it three times:

- In the first round we merely give some preliminary hints about some of these facets of number theory and their connection to each other.

- The second circuit will be a more precise run-through using the prime $p=691$ as an example. Here we will be highlighting six specific objects (or specific computations) occurring in the parts of number theory that correspond to each of our vertices. The fact that these six phenomena are related and that the linked chain that they form provides us with a powerful way of understanding each of them - and indeed constructing some of them - offers yet more evidence if we ever needed it that mathematics is an indivisible whole.

\footnotetext{
${ }^{3}$ It was fun to do that, and it was a particular pleasure to me; one of the joys of doing mathematics is that you have people like Ken Ribet as friends and colleagues.
} 
- After some discussion of background material we do the third lap around the hexagon, to formulate the Herbrand-Ribet Theorem for the general prime number $p$.

I hope that people who wish to get the general flavor of the number theory involved in this hexagon will be able to do so whether or not they work through some of the more detailed issues discussed in the later parts of this article.

Part II discusses results that give us interesting Galois representations that are (a) managed efficiently by knowledge of Frobenius eigenvalues and (b) arise from algebraic geometry.

Part III takes up these ideas more explicitly and discusses some of the issues that relate to what I call Ribet's "wrench".

Part IV hints at how the "Ribet philosophy", taken broadly, is continuing to inspire current work in the area.

The appendices deal with some of the finer structure of the packages of modular forms related to these questions.

PART I:

About the Herbrand-Ribet Theorem

\section{Cyclotomic Number Fields AND their ARITHMetic}

To launch into my topic, the "basic number fields" referred to in the title are the cyclotomic number fields. A cyclotomic number field is a field generated over the rational field $\mathbf{Q}$ by the adjunction of a primitive $N$ th root of unity, for some $N$. For example, we can view this field as the subfield of the field of complex numbers generated by $e^{2 \pi i / N}$.

The "first two" of these cyclotomic number field, i.e., $\mathbf{Q}\left(e^{2 \pi i / N}\right)$ for $N=3,4$, are thoroughly familiar to many mathematicians. They are the quadratic number fields $\mathbf{Q}(\sqrt{-3})$ and $\mathbf{Q}(\sqrt{-1})$ sitting nicely in the complex plane, and they have the property that their rings of integers represent elegantly symmetric lattices in the complex plane: for $N=3$ one gets a hexagonal lattice; for $N=4$, where the ring of integers in the cyclotomic field $\mathbf{Q}(\sqrt{-1})$ is the ring of Gaussian integers $\{a+i b \mid a, b \in \mathbf{Z}\}$, the corresponding lattice is the square lattice. The "next" cyclotomic field in turn, i.e., for $N=5$, also has had a great role to play in our subject in that it is a quadratic extension of the (quadratic) number field generated over $\mathbf{Q}$ by the golden mean; it is the field relevant for the classical construction of the regular pentagon.

But perhaps I should not be going - one by one - through the list of these cyclotomic number fields, for the totality of them have played a crucial role in the development of mathematics in general, and arithmetic in particular. Their importance was certainly recognized by Gauss, where the field extension $\mathbf{Q}\left(e^{2 \pi i / N}\right) / \mathbf{Q}$ was seen to be related to the construction of the regular $N$-gon, and to be a key to a fuller understanding of all quadratic number fields. By the latter part of the nineteenth century, thanks to the work of Kummer, it was known that the fine arithmetic features of these fields gave powerful methods to view (systematically) abelian extensions of number fields as generated by radicals, and to approach Fermat's Last Theorem for regular prime exponents. The modern arithmetic of

4 and some irregular 
cyclotomic fields per se is enriched by two fundamental theories, each with its own powerful viewpoints:

(1) The earlier of the two theories is Class Field Theory as initially established by Takagi, Artin, and Chevalley (cf. 11, 12]), which establishes a canonical identification between the Galois groups of abelian extensions of a number field $K$ and certain quotient groups of groups of fractional ideals in $K$. For more about this, see Chapter VII of [12] (and Subsection 4.1] below).

(2) The later theory is due to Iwasawa, who surmised a certain profound connection between:

- the structure of the ideal class group 5 - or equivalently, via Class Field Theory, of abelian everywhere unramified extensions - of cyclotomic number fields; and

- a $p$-adic interpolation of analytic number theory, more specifically, the $p$-adic version of Dirichlet $L$-functions as constructed by Kubota and Leopoldt6 (for more on this, see [74]).

In view of all this, one can see why Serge Lang once referred to the arithmetic theory of cyclotomic fields as the "backbone of algebraic number theory".

\section{SiX FACETS OF NUMBER THEORY}

Here again are the labels for the six topics.

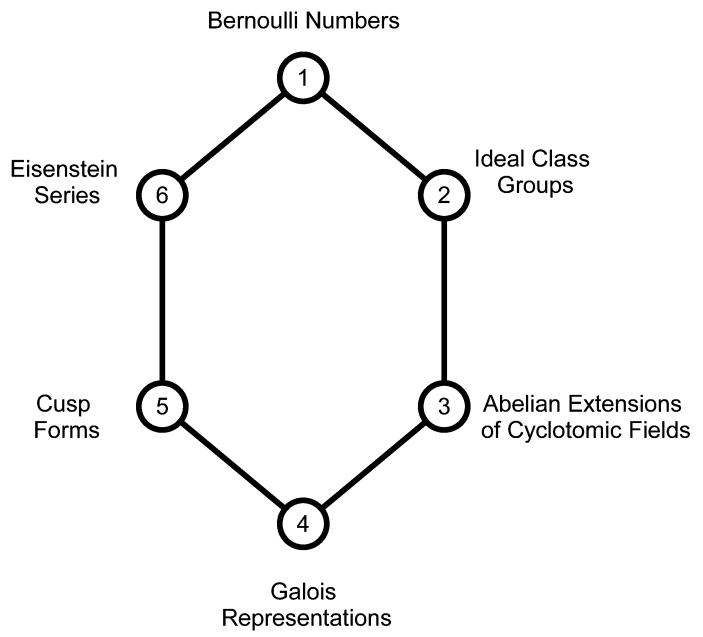

FIgURE 1. (repeated)

\footnotetext{
${ }^{5}$ More specifically, for $p$ a prime number, the $p$-primary components of the ideal class groups of cyclotomic fields are obtained by the extraction of $N$ th roots of unity, where $N$ is a power of $p$.

${ }^{6}$ The seeds for such a program were already planted in the latter part of the nineteenth century in the work of E. Kummer, and the foundations for this program occur in the work of Hensel.
} 
Here is a cartoon of how they get connected to each other.



Figure 2

In a sentence, Ken Ribet managed to pass from

- properties of Bernoulli numbers to

- note a consequence about Eisenstein series which

- allows him to construct certain cuspidal modular forms related to these Eisenstein series, and in turn to

- construct, using these cuspforms, a certain homomorphism of the automorphism group of $\overline{\mathbf{Q}}$, the field of algebraic numbers, to the group of upper triangular matrices in $\mathrm{GL}_{2}\left(\mathbf{F}_{p}\right)$ so that he may then

- form the number field $L \subset \overline{\mathbf{Q}}$ consisting of all the algebraic numbers fixed by the subgroup of automorphisms comprising the kernel of this homomorphism,

- this $L$ being the abelian extension of the cyclotomic field that was desired to be constructed and whose properties were to be verified,

thereby completing the circuit of Figure 2 .

\section{A FEW WORDS ABOUT SOME OF THE STATIONS AND HOW THEY ARE CONNECTED TO EACH OTHER}

4.1. Abelian extensions of number fields and ideal class groups. That there is a connection between these two arithmetic objects is seen already in Gauss's Disquisitiones Arithmeticae. It has subsequently been a theme threading through work of Dirichlet, Dedekind, Kummer, and Hilbert, leading to the more complete contemporary versions of Class Field Theory (see, e.g., [12, 1]).

For $K$ a number field, Class Field Theory offers us a construction of the category of abelian Galois extensions of $K$ in relatively concrete terms 7 An important

\footnotetext{
${ }^{7}$ Class Field Theory provides us with an isomorphism between the abelianization of $G_{K}$ and the profinite completion of the (group of connected components of the) idele class group of $K$. It is no accident, though, that abelian Galois extensions are more amenable to detailed study than more general Galois extensions, and this is not only because abelian groups are easier to study than more general groups. Just as, in algebraic topology, one must specify a base point to explicitly define the fundamental group of a connected space, but one need not do this to define its homology group, so too with a field $K$ one must specify a separable algebraic closure, $K^{\mathrm{sep}}$, of $K$ to explicitly define the full Galois group $G_{K}:=\operatorname{Gal}\left(K^{\text {sep }} / K\right)$. Without such a specification, $G_{K}$ is only defined "up to conjugation" (which is why it is particularly fitting to study the structure of $G_{K}$ via its linear representations, which are themselves only defined up to conjugation). But
} 
special case of this is the isomorphism that Class Field Theory provides, between $\operatorname{Gal}(H / K)$, the Galois group of the maximal abelian everywhere unramified Galois extension $H$ of $K$, and the ideal class group, $\mathcal{C l}(K)$, of $K$. A consequence of this isomorphism for us is that the problem of constructing quotient groups of the ideal class group of $K$ with certain properties translates to constructing abelian unramified extensions of $K$ with the corresponding properties. That is, it provides us with a link between stations 2 and 3 .

4.2. "Constructing" Galois extensions and Galois representations. Let us review the standard way of studying the cyclotomic field $L$ obtained by adjoining a primitive $N$ th root of unity to the rational field. This number field can be thought of as the splitting field of the polynomial $X^{N}-1$ over $\mathbf{Q}$. If $\mu_{N}$ denotes the group (under multiplication) of all $N$ th roots of unity in this splitting field, we have that $\mu_{N}$ is a cyclic group of order $N$, and it is the set of all roots of $X^{N}-1$. More precisely,

$$
X^{N}-1=\prod_{\zeta \in \mu_{N}}(X-\zeta) .
$$

If $G:=\operatorname{Gal}(L / \mathbf{Q})$ denotes the Galois group of $L$ over $\mathbf{Q}$, then $G$ preserves the set of roots of $X^{N}-1$ and thus can be thought of as a subgroup of the group of permutations of the set $\mu_{N}$. Even better, since the action of $G$ commutes with multiplication, $G$ acts as a group of automorphisms of the cyclic group $\mu_{N}$. So we get a natural imbedding

$$
\operatorname{Gal}(L / \mathbf{Q}) \hookrightarrow \operatorname{Aut}_{\mathrm{gp}}\left(\mu_{N}\right)=(\mathbf{Z} / N \mathbf{Z})^{*}=\mathrm{GL}_{1}(\mathbf{Z} / N \mathbf{Z}) .
$$

Note that the middle equality is given by the canonical isomorphism 8 of $(\mathbf{Z} / N \mathbf{Z})^{*}$ the group of units in the ring $\mathbf{Z} / N \mathbf{Z}$ - with the group of automorphisms of the cyclic group $\mu_{N}$. Thanks to a theorem of Gauss the first inclusion is an isomorphism, giving us one of the most venerable surjective Galois representations in the history of the subject:

$$
\omega_{N}: \operatorname{Gal}(L / \mathbf{Q}) \stackrel{\simeq}{\longrightarrow} \mathrm{GL}_{1}(\mathbf{Z} / N \mathbf{Z}),
$$

a faithful degree one representation of $\operatorname{Gal}(L / \mathbf{Q})$ over the $\operatorname{ring} \mathbf{Z} / N \mathbf{Z}$.

It is standard, nowadays, to construct Galois extensions over a field $K$ by finding natural continuous actions of $G_{K}:=\operatorname{Gal}\left(K^{\mathrm{sep}} / K\right)$ on vector spaces or on modules over rings, these coming to us usually as cohomology modules. Here $K^{\text {sep }}$ is a separable (algebraic) closure of $K$.

For example, if a module $H$, free of rank $d$ over a finite $\operatorname{ring} R$, is endowed with a continuous $R$-linear $G_{K}$-action, we would then get a representation

$$
\rho: G_{K} \rightarrow \operatorname{Aut}_{R}(H) \simeq \mathrm{GL}_{d}(R) .
$$

Call these things Galois representations (two such representations being viewed as equivalent if they can be brought, one to another, by conjugation with an element of $\left.\mathrm{GL}_{d}(R)\right)$. Since we have assumed that our ring $R$ is finite, the image of $\rho$ is finite,

one has no need to specify a separable algebraic closure of $K$ to define the abelianization of $G_{K}$,

$$
G_{K}^{\mathrm{ab}}:=G_{K} / G_{K}^{\prime}
$$

(here $G_{K}^{\prime}$ is the closed normal subgroup generated by commutators) whose quotients by closed subgroups of finite index provide us with the Galois groups of all finite abelian extensions of $K$. One can even "name" particular elements in $G_{K}^{\text {ab }}$ merely by giving appropriate data taken from the base field $K$.

${ }^{8}$ Associate to $\alpha \in(\mathbf{Z} / N \mathbf{Z})^{*}$ the automorphism $\zeta \mapsto \zeta^{\alpha}$ for $\zeta \in \mu_{N}$. 
and therefore there is a unique finite-degree Galois extension $L / K$ with $L \subset K^{\text {sep }}$ such that $\rho$ factors through the injective homomorphism

$$
G_{K} \rightarrow \operatorname{Gal}(L / K) \hookrightarrow \mathrm{GL}_{d}(R) .
$$

It is in this sense that our Galois representation $\rho$ has "constructed" $L / K$ (together with a faithful degree $d$ representation of $\operatorname{Gal}(L / K)$ over the ring $R)$.

When we get to station 4 of Figure 1 we'll be touching on such constructions again, in particular, in Section 9 below.

4.3. Abelian extensions and two-dimensional Galois representations. Our desired abelian extension $L$ (as in station 3 in Figure 1) will in fact be cyclic of degree $p$ over the cyclotomic number field $\mathbf{Q}\left(e^{2 \pi i / p}\right)$, but decidedly non-abelian over $\mathbf{Q}$, the field of rational numbers. Although it might seem odd at first glance, it is natural to construct these extensions by first constructing a non-abelian (twodimensional) indecomposable representation of $G_{\mathbf{Q}}$, the Galois group of an algebraic closure of $\mathbf{Q}$, into $\mathrm{GL}_{2}\left(\mathbf{F}_{p}\right)$. One then gets the required extension field as the field cut out by this degree two representation over $\mathbf{F}_{p}$, in the manner discussed in Subsection 4.2 above. This type of argument will be giving us a link between stations 3 and 4 .

4.4. Modular forms and the two-dimensional Galois representations that are associated to them. Cuspform 9 come into play, for they are a very convenient source of irreducible continuous two-dimensional $G_{\mathbf{Q}}$ representations over the field of $p$-adic numbers, $\mathbf{Q}_{p}$, and over finite degree extensions of $\mathbf{Q}_{p}$, for any prime $p$. For some introductory discussion about this, see Sections 7 and 8 below. For a slightly more descriptive discussion of the passage from cuspforms to Galois representations via Deligne's Theorem, see Section 9 .

By suitably reducing these $G_{\mathbf{Q}}$ representations to characteristic $p$, one gets a supply of continuous two-dimensional $G_{\mathbf{Q}}$ representations over finite fields $k$ of characteristic $p$; for $p>2$ all the representations so obtained have the further property that the complex conjugation involution does not act as a scalar. The conjecture of Serre ([59]), recently proved by Khare and Wintenberger (see [36], 33], [35]) asserts that every irreducible continuous two-dimensional $G_{\mathbf{Q}}$ representation over a finite field in which complex conjugation involution does not act as a scalar comes, in this manner, from a cuspform 10

4.5. The Ribet wrench: first encounter. Ribet's goal is to find certain reducible-but-indecomposable two-dimensional Galois representations over finite fields, say over $\mathbf{F}_{p}$. By choosing a suitable basis of the two-dimensional $\mathbf{F}_{p}$-vector space, such a representation can be given by a homomorphism

$$
\begin{aligned}
G_{\mathbf{Q}} \rightarrow \text { upper triangular matrices } & \subset \mathrm{GL}_{2}\left(\mathbf{F}_{p}\right), \\
g & \mapsto\left(\begin{array}{cc}
\chi_{1}(g) & b(g) \\
0 & \chi_{2}(g)
\end{array}\right),
\end{aligned}
$$

\footnotetext{
${ }^{9}$ More specifically, in this article we will only be interested in eigenforms for the Hecke operators. For a fine introduction to this material and its connection to $L$-functions and modular curves, see Rohrlich's paper [52]. For an introduction to the subject that focuses on computation, see [67.

${ }^{10}$ For an introductory survey of Serre's Conjecture and related material, see [51].
} 
where $\chi_{1}$ and $\chi_{2}$ are characters of the Galois group with values in $\mathbf{F}_{p}^{*}$. More succinctly we evoke such a representation by the following matrix picture:

$$
\left(\begin{array}{cc}
\chi_{1} & * \\
0 & \chi_{2}
\end{array}\right) \text {. }
$$

The intersection of the kernels of these two characters defines, in the usual manner of Galois theory, an abelian field extension $M$ of the rational field $\mathbf{Q}$; that is, $M$ is the smallest subfield of the algebraic closure $\overline{\mathbf{Q}}$ such that the restriction of those characters to the subgroup $\operatorname{Gal}(\overline{\mathbf{Q}} / M) \subset \operatorname{Gal}(\overline{\mathbf{Q}} / \mathbf{Q})$ is trivial. Thus $M$ is the smallest field extension for which the above representation, when restricted to $\operatorname{Gal}(\overline{\mathbf{Q}} / M)$, has the shape

$$
\left(\begin{array}{ll}
1 & * \\
0 & 1
\end{array}\right)
$$

In the theory we are presenting, the field $M$ is usually the $p$-cyclotomic field $\mathbf{Q}\left(e^{2 \pi i / p}\right)$.

If the representation restricted to $\mathrm{Gal}\left(\overline{\mathbf{Q}} / \mathbf{Q}\left(e^{2 \pi i / p}\right)\right)$ is nontrivial, then the image of $\operatorname{Gal}\left(\overline{\mathbf{Q}} / \mathbf{Q}\left(e^{2 \pi i / p}\right)\right)$ in $\mathrm{GL}_{2}\left(\mathbf{F}_{p}\right)$ is isomorphic to the group of unipotent upper triangular matrices displayed above, and hence is a cyclic group of order $p$ cutting out a cyclic Galois extension $N / \mathbf{Q}\left(e^{2 \pi i / p}\right)$. So if we have an explicit understanding of the initial two-dimensional representation of $G_{\mathbf{Q}}$, then we have, in similar explicit terms, "constructed" the cyclic degree $p$ extension $N$ of the $p$-cyclotomic field.

Ribet achieves this aim by the following.

- Initially, he finds an irreducible two-dimensional Galois representation over a field of characteristic 0 , say over the field $\mathbf{Q}_{p}$ of $p$-adic numbers, that is, a continuous homomorphism

$$
\rho: G_{\mathbf{Q}} \rightarrow \operatorname{Aut}_{\mathbf{Q}_{p}}(V) \approx \mathrm{GL}_{2}\left(\mathbf{Q}_{p}\right),
$$

where $V$ is a two-dimensional $\mathbf{Q}_{p}$-vector space, and the action of $G_{\mathbf{Q}}$ preserves no line.

- He then appropriately reduces this Galois representation modulo $p$. To do this, though, he must choose a $\mathbf{Z}_{p}$-lattice $\Omega \subset V$ that is stabilized by the action of $G_{\mathbf{Q}}$ (such a lattice always exists) and then pass to the action of $G_{\mathbf{Q}}$ on $\bar{V}:=\Omega / p \Omega$.

In this manner you get a representation mod $p$, which we will call the residual representation obtained from the lattice $\Omega$ :

$$
\bar{\rho}: G_{\mathbf{Q}} \rightarrow \operatorname{Aut}_{\mathbf{F}_{p}}(\bar{V}) \approx \mathrm{GL}_{2}\left(\mathbf{F}_{p}\right) .
$$

A priori, the equivalence class of the representation $\bar{\rho}$ depends on the choice of lattice $\Omega$. Now, there are many such lattices $\Omega$ that are stabilized by the $G_{\mathbf{Q}^{-}}$action, so we need to discuss how many inequivalent representations $\bar{\rho}$ might be obtained by varying the choice of $\Omega$. For a start, multiplying any such lattice by a nonzero scalar provides another stable lattice, but it is evident that the corresponding residual representations of lattices that differ by a scalar change are isomorphic.

There are, however, situations where the residual representation may change (a bit) depending upon the lattice $\Omega$ that is chosen. What is independent of the lattice chosen is the semisimplification of the residual representation $\bar{\rho}$. In particular, if the residual representation associated to one lattice stabilized by the action of $G_{\mathbf{Q}}$ is reducible, then it is so for all 
such lattices, and the two one-dimensional representations, i.e., characters, into which the two-dimensional residual representation decomposes are independent of the lattice chosen. But the two-dimensional reducible residual representation $\bar{\rho}$ itself may indeed depend upon the lattice.

- The key to Ribet's construction is to start with representations $\rho$ that are irreducible, and yet have residual representations that are reducible; that is, in the terminology of the discussion above, the residual representations have matrix pictures looking like

$$
\left(\begin{array}{cc}
\chi_{1} & * \\
0 & \chi_{2}
\end{array}\right),
$$

where, again as in the discussion above, one has constructed a cyclic $p$ extension if the corresponding residual representation, when restricted to $\operatorname{Gal}(\overline{\mathbf{Q}} / M)$, that has the shape

$$
\left(\begin{array}{ll}
1 & * \\
0 & 1
\end{array}\right)
$$

is nontrivial.

- In the above situation one can always change the lattice $\Omega$ suitably 11 to guarantee that the above representation is nontrivial.

- The irreducibility of his initial representation is crucial to his method. In its later manifestations this type of strategy can be framed as follows: you are looking for a "somewhat degenerate object" (e.g., a reducible representation) and you hope to get it as a "degenerate" member of, say, a one-parameter family of nondegenerate objects (e.g., irreducible representations). We will be calling such reducible representations that are obtained as limits of irreducible ones liminal.

This manner of reasoning is how one will be passing from station 4 to station 3.

4.6. Herbrand's Theorem; regular primes, properly irregular primes, and improperly irregular primes. A consequence of the Herbrand Theorem is that if an odd prime number $p$ does not divide (the numerators of) any of the Bernoulli numbers $B_{2 k}$ for $2 k=2,4, \ldots, p-3$, then $p$ does not divide the class number of the cyclotomic field $\mathbf{Q}\left(e^{2 \pi i / p}\right)$. The bare bones of the argument for this is the following: first one constructs a specific element $\theta$ in the integral group ring of the Galois group $\operatorname{Gal}\left(\mathbf{Q}\left(e^{2 \pi i / p}\right) / \mathbf{Q}\right)$ that annihilates the $p$-primary component of the ideal class group of $\mathbf{Q}\left(e^{2 \pi i / p}\right)$, and then one shows that under the hypotheses above, this element $\theta$ is a unit in the integral group ring. See Chapter 6 of Larry Washington's Introduction to Cyclotomic Fields [74].

A prime number $p$ that does not divide the class number of the cyclotomic field $\mathbf{Q}\left(e^{2 \pi i / p}\right)$ is called a regular prime. It is known that there are infinitely many irregular primes. But even now, after much thought has been devoted to this area of mathematics, it is unknown whether or not there are infinitely many regular primes. For the story of conjectures regarding this (and conjectural ideas about the related statistics), see 74. If a prime $p$ is irregular, but does not divide the class number of the maximal totally real subfield, $\mathbf{Q}\left(e^{2 \pi i / p}+e^{-2 \pi i / p}\right)$, of $\mathbf{Q}\left(e^{2 \pi i / p}\right)$, then $p$ is called properly irregular. A conjecture of Vandiver ([73; see also Section

\footnotetext{
${ }^{11}$ I envision it as a kind of "wrenching action".
} 
10.3 in [74]) is that all irregular primes are properly irregular. Vandiver's conjecture has the advantage of being verified for $p<163,000,000$ ([10]; also compare with computations for $p<12,000,000$ [9]) and has strong consequences, so even if there exist improperly irregular primes, proper irregularity is a useful condition to bear in mind. What is of interest, specifically for our story, is that the existence of the Galois extensions constructed by Ribet was already known to be true for any properly irregular prime $p$. For such properly irregular primes, the sought-for extensions can be generated over $\mathbf{Q}\left(e^{2 \pi i / p}\right)$ by adjoining $p$ th roots of appropriate cyclotomic units 12 But even for these primes, the "construction method" we are describing illuminates the landscape: it reveals a deep connection between seemingly disparate mathematical objects.

\section{Rounding the CiRCuit With The PRIME 691}

Here is a taste of the "six facets" discussed above, as they manifest themselves for the prime 691. Since 691 is properly irregular, as mentioned above, the bald existence of a Galois extension with the properties that Ribet's theorem guarantees is not at issue: the desired Galois extension has been known to exist for quite a long time. Even more to the point - as we shall get to, later - for this very example, Serre had suggested exactly where to find the desired Galois extension, and unpublished work of Greenberg and Monsky did indeed find it, and in essentially the same context that we will be reviewing, all this happening before Ribet's work.

Here, rather, we will have our sights on Ribet's general method of construction for all (irregular) primes $p$, but we concentrate on this well-studied $p=691$. We will make the circuit, and for each of the six vertices of our hexagon we will be signaling an explicit piece of number theory - a computation - related to that vertex. These six phenomena are, as we indicated in the introduction, essentially linked together.

1 Divisibility of the numerator of Bernoulli numbers modulo $p$. Let $B_{k}$ be the $k$ th Bernoulli number as in Jacob Bernoulli's Ars Conjectandi 13 For odd integers $k>1, B_{k}$ vanishes. For even integers $2 k$ the Bernoulli number $B_{2 k}$ is the coefficient of $x^{2 k} / 2 k$ ! in the power series expansion

$$
\frac{x}{e^{x}-1}=1-\frac{x}{2}+\sum_{k=1}^{\infty}(-1)^{k+1} B_{2 k} \frac{x^{2 k}}{2 k !} .
$$

We moderns have (as Bernoulli himself also had) easy methods for its computation, and here is a list of the first few $\frac{B_{2 k}}{4 k}$ :

$$
\begin{gathered}
B_{2} / 4=+1 / 24, \\
B_{4} / 8=-1 / 240, \\
B_{6} / 12=+1 / 504, \\
B_{8} / 16=-1 / 480, \\
B_{10} / 20=+1 / 264,
\end{gathered}
$$

\footnotetext{
${ }^{12}$ See footnote 16 below. For a discussion (by Joe Buhler and David Harvey) of the likeliness of anyone being able to find (in our lifetime) an example of an improperly irregular prime - even if Vandiver's conjecture is false - see Appendix II below.

${ }^{13}$ An English translation of this extraordinary work has recently been published [7] with extensive introductory material.
} 
which might lead us to make a rash conjecture about the numerator of these numbers, if not for the next case,

$$
B_{12} / 24=-691 / 65520 .
$$

The prime $p=691$, then, divides the numerator of $B_{12} / 24$. Hold that thought!

2 The ideal class group taken modulo $p$ of the cyclotomic field $\mathbf{Q}\left(e^{2 \pi i / p}\right)$. For a number field $K$ the set of nonzero ideals in its ring of integers, viewed with the multiplicative structure that it naturally has (multiplication of ideals), but taken modulo the equivalence relation generated by requiring that any principal ideal is trivial, forms a finite abelian group, called the ideal class group of $K$.

We keep to $p=691$, but in the body of this discussion we will revert to denoting 691 simply by the letter $p$. Take $K$ to be the cyclotomic field $\mathbf{Q}\left(e^{2 \pi i / p}\right)$. The automorphism group of this field, that is, the Galois group $\operatorname{Gal}\left(\mathbf{Q}\left(e^{2 \pi i / p}\right) / \mathbf{Q}\right)$, is canonically isomorphic to $\mathbf{F}_{p}^{*}$, the multiplicative group of the prime field of characteristic $p$. Denote this canonical isomorphism by

$$
\iota: \operatorname{Gal}\left(\mathbf{Q}\left(e^{2 \pi i / p}\right) / \mathbf{Q}\right) \longrightarrow \mathbf{F}_{p}^{*}
$$

Form $X:=$ the ideal class group of $K$ modulo $p(=691)$. The finite abelian group $X$ has a natural action of $\operatorname{Gal}\left(\mathbf{Q}\left(e^{2 \pi i / p}\right) / \mathbf{Q}\right)$, which we denote $(\alpha, x) \mapsto \alpha(x)$ for $\alpha \in \operatorname{Gal}\left(\mathbf{Q}\left(e^{2 \pi i / p}\right) / \mathbf{Q}\right)=\mathbf{F}_{p}^{*}$ and $x \in X$.

What is the abelian group $X$ (which is convenient to think of as an $\mathbf{F}_{p}$-vector space), and what is this action?

The answer is that $X$ is nontrivial 14 Hold that thought!

In passing, we might note that the nontriviality of $X$ already signals the elementary strategy for proving Fermat's Last Theorem - the method used for the prime exponent 3 by Euler 15

The group $X$ viewed as vector space over $\mathbf{F}_{p}$ is, in fact, of dimension two, and has a basis $\{x, y\}$ of eigenvectors for the action of

$$
\operatorname{Gal}\left(\mathbf{Q}\left(e^{2 \pi i / p}\right) / \mathbf{Q}\right) \stackrel{\iota}{\longrightarrow} \mathbf{F}_{p}^{*}
$$

such that for $\alpha \in \operatorname{Gal}\left(\mathbf{Q}\left(e^{2 \pi i / p}\right) / \mathbf{Q}\right)$ we have the formulas

$$
\begin{aligned}
& \alpha(x)=\iota(\alpha)^{691-12} \cdot x=\iota(\alpha)^{-11} \cdot x, \\
& \alpha(y)=\iota(\alpha)^{691-200} \cdot y=\iota(\alpha)^{-199} \cdot y .
\end{aligned}
$$

Though both of these "lines" in the two-dimensional $\mathbf{F}_{p}$ vector space $X$ are interesting, the discussion about each of them is somewhat similar, so for specificity we sometimes concentrate, below, a bit more on the line $x \cdot \mathbf{F}_{p}$ in $X$ admitting an action by $\operatorname{Gal}\left(\mathbf{Q}\left(e^{2 \pi i / p} / \mathbf{Q}\right)\right.$ via the character $\iota^{-11}$.

\footnotetext{
${ }^{14} \mathrm{~A}$ natural impulse, then, is to seek a construction of specific nonprincipal ideals whose $p$ th powers are principal. This is, in effect, elegantly done for us, somehow, by the Herbrand-Ribet Theorem both in our particular case $(p=691)$ and (the analogous task is done) in the general case of irregular primes $p$.

${ }^{15}$ Ernst Kummer already understood the deep arithmetic consequences that follow from knowledge of the vanishing of the ideal class group modulo $p$ of the cyclotomic number field $\mathbf{Q}\left(e^{2 \pi i / p}\right)$. (If this happens, then "enough" of the fundamental theorem of arithmetic holds in the ring of integers of $\mathbf{Q}\left(e^{2 \pi i / p}\right)$ to allow one to prove that Fermat's Last Theorem for the equation $x^{p}+y^{p}=z^{p}$ will not work for the exponent 691.)
} 
3 Abelian unramified extensions of degree 691 over the cyclotomic field $K=\mathbf{Q}\left(e^{2 \pi i / 691}\right)$. There are field extensions $L / K$ that are (cyclic) of degree 691 , that are everywhere unramified and that have the further property that $L / \mathbf{Q}$ is Galois 16 and, furthermore, there are precisely two such field extensions $L / K$; call them $L^{\{12\}}$ and $L^{\{200\}}$. These two extensions are distinguished by the nature of the action by conjugation; that is, by the conjugation-action of any lifting $\tilde{\alpha}$ of $\alpha \in \operatorname{Gal}(K / \mathbf{Q})$ to $\operatorname{Gal}(L / \mathbf{Q})$ on elements $\gamma \in \operatorname{Gal}(L / K) \subset \operatorname{Gal}(L / \mathbf{Q})$. This conjugation-action is given for $L=L^{\{12\}}$ by the formula

$$
\tilde{\alpha} \gamma \tilde{\alpha}^{-1}=\iota(\alpha)^{691-12} \cdot \gamma=\iota(\alpha)^{-11} \cdot \gamma,
$$

and for $L=L^{\{200\}}$ it is given by

$$
\tilde{\alpha} \gamma \tilde{\alpha}^{-1}=\iota(\alpha)^{691-200} \cdot \gamma=\iota(\alpha)^{-199} \cdot \gamma .
$$

4 Galois representations into $\mathrm{GL}_{2}\left(\mathbf{F}_{691}\right)$. For each of these two extensions $L / \mathbf{Q}$ (i.e., $L=L^{\{12\}}$ or $L^{\{200\}}$ ) the equations above allow us to view $\operatorname{Gal}(L / \mathbf{Q})$ as a semidirect product

$$
\operatorname{Gal}(L / \mathbf{Q})=\operatorname{Gal}(L / K) \rtimes \operatorname{Gal}(K / \mathbf{Q}) .
$$

From each of these extensions $L / \mathbf{Q}$ we will now describe corresponding two-dimensional representations of the Galois group of the rational field $\mathbf{Q}$, and, as we shall see, these "Galois representations" deserve our attention.

For example, take $L:=L^{\{12\}}$. Let $\psi: \operatorname{Gal}(L / K) \simeq \mathbf{F}_{p}^{+}$be a choice of isomorphism. Then form the representation

$$
\bar{\rho}: \operatorname{Gal}(L / \mathbf{Q}) \longrightarrow \mathrm{GL}_{2}\left(\mathbf{F}_{p}\right)
$$

by sending $\gamma \cdot \alpha$ to

$$
\left(\begin{array}{cc}
1 & \psi(\gamma) \\
0 & \iota(\alpha)^{11}
\end{array}\right)
$$

The displayed equations above show that this is indeed a homomorphism. Working similarly with $L^{\{200\}}$ in this way, we get two Galois representations for our two

\footnotetext{
${ }^{16}$ Kummer knew that for $p$ a prime number and for any field $F$ of characteristic different from $p$ that contained a primitive $p$ th root of unity, the cyclic field extensions of $F$ of degree $p$ are obtained by extracting $p$ th roots of elements of $F$; moreover, these cyclic field extensions are in one-to-one correspondence with the $\mathbf{F}_{p}$-lines in the $\mathbf{F}_{p}$-vector space $F^{*} /\left(F^{*}\right)^{p}$ (although he would express this knowledge via a completely different vocabulary). So, for example, there are infinitely many cyclic extensions of degree 691 over the cyclotomic field $K=\mathbf{Q}\left(e^{2 \pi i / 691}\right)$. One way, then, of pinpointing the everywhere unramified cyclic extensions of degree 691- there being exactly two such extensions that are Galois over $\mathbf{Q}$ - would be to exhibit elements $v_{1}, v_{2}$ in $K^{*}=\mathbf{Q}\left(e^{2 \pi i / 691}\right)^{*}$ such that the field extensions obtained by extracting a 691th root of these elements are the ones you want. This can be done in the case $p=691$. It can be done more generally for properly irregular primes $p$; moreover, in the properly irregular case the elements whose $p$ th roots generate the sought-for everywhere unramfied extensions can be taken to be cyclotomic units. For a discussion of the basic theory behind all this, see 74] and specifically read about cyclotomic units in Section 1 in Chapter 8 (especially Theorem 8.2), and read about the so-called Reflection Theorems and their consequences for Vandiver's conjecture in 10.2, 10.3 of loc. cit. For a one-page recollection of this theory, see http://mathoverflow.net/questions/37880/kummer-generator-forthe-ribet-extension/37951\#37951. As we shall see, however, the method we are discussing, which works for the full class of irregular primes, whether they be properly irregular or not, does not exhibit these extensions in that way.
} 
choices of $L$; call them $\rho^{\{12\}}$ and $\rho^{\{200\}}$, respectively. The equivalence class 17 of each of these representations $\bar{\rho}$ is independent of our choices.

Remember these representations!

5 The cuspform $\Delta$ of level 1 and weight 12 . I am referring to that very intensely studied infinite product

$$
\Delta(q)=q \prod_{n=1}^{\infty}\left(1-q^{n}\right)^{24}=0+\sum_{n=1}^{\infty} \tau(n) q^{n}
$$

which can be directly thought of as it is presented here, namely, a power series in the variable $q$; or, putting $q=e^{2 \pi i z}$ we may view it as an analytic function of the variable $z$ in the upper half-plane, where it is shown to satisfy the symmetry $\Delta(-1 / z)=z^{12} \Delta(z)$ (i.e., $\Delta$ is a modular form of level 1 and weight 12). Its Fourier series coefficients, $\tau(n)$ (for $n=1,2, \ldots$ ), have been brilliantly analyzed by generations of mathematicians, including Ramanujan. Simple recurrence relations (first described by Mordell) allow you to "retrieve" the Ramanujan "tau"-function $n \mapsto \tau(n)$ from the values $\ell \mapsto \tau(\ell)$ for all prime numbers $\ell$.

A congruence due to Ramanujan (47) 18 gives us that

$$
\tau(n) \equiv \sum_{0<d \mid n} d^{11} \bmod 691
$$

for every positive integer $n$. In particular,

$$
\tau(\ell) \equiv 1+\ell^{11} \bmod 691
$$

for every prime number $\ell$.

Remember this!

There is also a unique cuspform - call it $\Delta^{\{200\}}$ - of level 1 and weight 200 with Fourier coefficients $n \mapsto \tau^{\{200\}}(n) \in \mathbf{Q}_{691}$ that enjoys a similar congruence 19

$$
\tau^{\{200\}}(n) \equiv \sum_{0<d \mid n} d^{199} \bmod 691
$$

for every positive integer $n$. In particular,

$$
\tau^{\{200\}}(\ell) \equiv 1+\ell^{199} \bmod 691
$$

for every prime number $\ell$.

\footnotetext{
${ }^{17}$ As previously mentioned, for any commutative ring with unit, $R$, and any group $G$, two homomorphisms $h_{1}, h_{2}: G \rightarrow \mathrm{GL}_{N}(R)$ are said to define equivalent representations if there is an element $\gamma \in \mathrm{GL}_{N}(R)$ such that $h_{2}$ is the composition of $h_{1}$ with the automorphism of $\mathrm{GL}_{N}(R)$ given by conjugation by $\gamma$.

${ }^{18}$ For a list of similar congruences that the Fourier coefficients of $\Delta$ satisfy modulo powers of the primes 2, 3, 5, 7 and modulo 23 (which, along with 691 compose the set of "exceptional primes" for $\Delta$ ), see 68 .

${ }^{19}$ I am thankful to William Stein for computing this: he tells me that the Fourier coefficients $\left\{\tau^{\{200\}}(n)\right\}_{n}$ generate a field of degree 16 over $\mathbf{Q}$ and the primes dividing 691 in this field have degrees $1,1,2,2$, and 10; completion at one of those primes of degree 1 gives us the cuspform $\Delta^{\{200\}}$ with Fourier coefficients in $\mathbf{Q}_{691}$ enjoying the congruences we are describing.
} 
6 The Eisenstein series $E_{12}$ of level 1 and weight 12 , modulo $p=691$.

The Fourier expansion of this modular form is given by

$$
E_{12}(q)=-B_{12} / 24+\sum_{n=1}^{\infty}\left(\sum_{0<d \mid n} d^{11}\right) q^{n} .
$$

As we have mentioned in $\mathbf{1}, B_{12} / 24=-691 / 65520$ (i.e., we are coming full circle). So $B_{12} / 24 \equiv 0$ modulo 691 and therefore our Eisenstein series has zero as its constant term modulo 691. Since, as we have mentioned in $\mathbf{5}$,

$$
\tau(n) \equiv \sum_{0<d \mid n} d^{11} \bmod 691,
$$

we get that the Eisenstein series $E_{12}$ has exactly the same Fourier expansion, modulo 691 , as $\Delta$.

Similarly, the Eisenstein series $E_{200}$ of level 1 and weight 200 given by

$$
E_{200}(q)=-B_{200} / 400+\sum_{n=1}^{\infty}\left(\sum_{0<d \mid n} d^{199}\right) q^{n}
$$

has the property that the numerator of $-B_{200} / 400$, its constant term 20 is divisible by the prime 691 . So, again, the Fourier series of the Eisenstein series $E_{200}$ taken modulo 691 has zero as its constant term 21

The Herbrand-Ribet Theorem assures us that these six arithmetic events are not mere coincidences, and that there are implications of a very similar sort going all the way around the hexagon for any prime number $p$.

\section{ROUNDING THE CIRCUIT AGAIN: \\ A statement of the Herbrand-Ribet Theorem}

Theorem 1 (Herbrand-Ribet). Let $p$ be a prime number and $2 k$ an even integer greater than 2 and less than $p-1$. The following are equivalent:

1 The numerator of the Bernoulli number $\frac{B_{2 k}}{4 k}$ is divisible by $p$. In Kummer's terminology, this is what is meant by $p$ being an irregular prime number 22

6 The constant term in the Fourier expansion of the Eisenstein series $E_{2 k}$ of weight $2 k$ and level 1 is congruent to zero modulo $p$. (Colloquially we can say, the Fourier expansion of $E_{2 k}$ "looks cuspidal modulo $p$ ".)

5 For some-or, equivalently, for every-integer $2 \leq w<\infty$ there exist the following objects:

- A number field $F_{w} \subset \overline{\mathbf{Q}}$. Let $\mathcal{O}_{w} \subset F_{w}$ denote the ring of integers in $F_{w}$.

- A prime ideal $P_{w} \subset \mathcal{O}_{w}$ such that $\mathcal{O}_{w} / P_{w}=\mathbf{F}_{p}$.

\footnotetext{
${ }^{20}$ If you're curious, it is $389 \cdot 691 \cdot 5370056528687$ times this 204-digit prime number: $3452690329392158031464109281736967404068448156842396721012992064214519445919256941 \sim$ $5445652760676623601087497272415557084252765272786877636295951962087273561220060103 \sim$ 6506871681124610986596878180738901486527.

${ }^{21}$ In fact, $E_{200}$ has exactly the same Fourier expansion, modulo 691 , as a certain cuspform of weight 200 , which we called $\Delta^{\{200\}}$, has. This is meant in the sense that the Fourier coefficients of $\Delta^{\{200\}}$ lie in the ring of integers of a number field that has a degree one prime ideal whose residue field is $\mathbf{F}_{691}$; when reduced modulo this prime ideal, one has $\Delta^{\{200\}} \equiv E_{200}$.

${ }^{22}$ Therefore, as someone mentioned after my lecture, in contrast to what happens in elementary geometry, our hexagon is only of special interest when it is irregular.
} 
- A power series

$$
\Phi_{w}=0+q+\sum_{n=2}^{\infty} t_{w}(n) q^{n}
$$

with coefficients $t_{w}(n) \in \mathcal{O}_{w}$, such that

- when viewed as a power series in $q=e^{2 \pi i z}$ with coefficients in $\mathbf{C}$ via any imbedding $F_{w} \hookrightarrow \mathbf{C}$, this power series is the Fourier series of a cuspidal eigenform (alias newform) on $\Gamma_{1}(p)$ of weight $w$;

- when reduced modulo the prime ideal $P_{w}$ and viewed as a power series with coefficients in the prime field $\mathbf{F}_{p}$, we have, for each integer $n \geq 0$, the congruence

$$
t_{w}(n) \equiv \sum_{0<d \mid n} d^{2 k-1} .
$$

In more colloquial vocabulary, the Fourier expansion of $\Phi_{w}$ is congruent to the Fourier expansion of $E_{2 k}$ modulo $p$.

4 There exist the following objects:

- A number field $F \subset \overline{\mathbf{Q}}$. Let $\mathcal{O} \subset F$ denote the ring of integers in $F$.

- A prime ideal $P \subset \mathcal{O}$ such that $\mathcal{O} / P=\mathbf{F}_{p}$. Let $\mathcal{O}_{P} \subset F_{P}$ be the completion of $\mathcal{O} \subset F$ at $P$. Let $\pi_{P} \in \mathcal{O}_{P}$ denote a uniformizer of the discrete valuation $\operatorname{ring} \mathcal{O}_{P}$.

- An abelian variety $A$ defined over $\mathbf{Q}$ with the following properties:

- The ring of integers $\mathcal{O}$ acts as a ring of endomorphisms of $A$ over $\mathbf{Q}$.

- Letting $A\left[P^{\nu}\right] \subset A(\overline{\mathbf{Q}})$ denote the intersection of the kernels of all the endomorphisms of $A$ that lie in the ideal $P^{\nu}$ and noting that $\pi_{P}$ : $A\left[P^{\nu+1}\right] \rightarrow A\left[P^{\nu}\right]$ is a surjective homomorphism, consider the projective system

$$
\cdots \rightarrow A\left[P^{\nu+1}\right] \rightarrow A\left[P^{\nu}\right] \rightarrow \cdots A[P]
$$

The projective limit $T_{P}(A):=\lim _{\nu} A\left[P^{\nu}\right]$ has a natural $\mathcal{O}_{P}\left[G_{\mathbf{Q}}\right]$-action. Form the $F_{P}$-vector space $V:=V_{P}(A)=T_{P}(A) \otimes_{\mathcal{O}_{P}} F_{P}$. This $F_{P}$ vector space is of dimension two.

- There is an $\mathcal{O}_{P}$-lattice $23 \Omega \subset$ stable under the action of $G_{\mathbf{Q}}$ for which the representation

$$
G_{\mathbf{Q}} \longrightarrow \operatorname{Aut}\left(\Omega / \pi_{P} \Omega\right) \cong \mathrm{GL}_{2}\left(\mathbf{F}_{p}\right)
$$

is indecomposable but reducible and whose semisimplification consists of two characters,

$$
\mathbf{1}, \omega_{p}^{2 k-1}: G_{\mathbf{Q}} \longrightarrow \mathrm{GL}_{1}\left(\mathbf{F}_{p}\right)=\mathbf{F}_{p}^{*},
$$

where 1 is the trivial character and $\omega_{p}$ is the basic p-cyclotomic character introduced in Subsection 4.2 above.

- A has good reduction at all primes different from $p$.

\footnotetext{
${ }^{23}$ A finitely generated $\mathcal{O}_{P}$-submodule $\Omega \subset V$ is called an $\mathcal{O}_{P}$-lattice if the natural $\mathcal{O}_{P^{-}}$ homomorphism

$$
\Omega \otimes \mathcal{O}_{P} F_{P} \rightarrow V
$$

is an isomorphism.
} 
- A achieves good reduction at all primes when the base field is extended to the maximal real subfield $\mathbf{Q}\left(e^{2 \pi i / p}+e^{-2 \pi i / p}\right)$ in the $p$-cyclotomic field.

3 Let $K$ be the cyclotomic field $K=\mathbf{Q}\left(e^{2 \pi i / p}\right)$. There is a field extension $L / K$ that is cyclic of degree $p$, that is everywhere unramified, and that has the further property that $L / \mathbf{Q}$ is Galois, and furthermore the action by conjugation of any lifting $\tilde{\alpha}$ of $\alpha \in \operatorname{Gal}(K / \mathbf{Q})$ to $\operatorname{Gal}(L / \mathbf{Q})$ on any element $y \in \operatorname{Gal}(L / K) \subset \operatorname{Gal}(L / \mathbf{Q})$ is given by the formula

$$
\tilde{\alpha} y \tilde{\alpha}^{-1}=\iota(\alpha)^{p-2 k} \cdot y=\iota(\alpha)^{1-2 k} \cdot y .
$$

2 There is a cyclic subgroup $X$ of order $p$ in the ideal class group of $K=$ $\mathbf{Q}\left(e^{2 \pi i / p}\right)$ stabilized by the action of the Galois group $\iota: \operatorname{Gal}\left(\mathbf{Q}\left(e^{2 \pi i / p}\right) / \mathbf{Q}\right) \simeq \mathbf{F}_{p}^{*}$ and such that the action of $\alpha \in \operatorname{Gal}\left(\mathbf{Q}\left(e^{2 \pi i / p}\right) / \mathbf{Q}\right)$ on $X$ is given by the formula

$$
\alpha(x)=\iota(\alpha)^{p-2 k} \cdot x=\iota(\alpha)^{2 k-1} \cdot x .
$$

We end Part I of this article by accompanying these statements in their counterclockwise route with some brief discussions; a few of these points will be expanded upon in slightly more detail in the later sections.

- $1 \Rightarrow 6$.

This is just because $\frac{B_{2 k}}{4 k}$ is the constant term of the Fourier expansion of $E_{2 k}$.

- 6 . 5 .

Here one depends upon the algebraic-geometric interpretation of modular forms - as sections of bundles over algebraic curves. The question of lifting a $\bmod p$ form to characteristic zero is then dealt with by standard exact sequences, very much helped by the fact that we are over algebraic curves. (The analogous procedure, when one deals with higher rank automorphic forms, is not so smooth-going.) Once one lifts, standard methods allow one to obtain a lift that is an eigenform, i.e., is "eigen" for all the Hecke operators that one needs.

A curious point is that we have our choice of infinitely many different newforms $f$ (at least one for each weight). Ken chose to work with cuspidal newforms of weight two. In the next step we will be making use of Galois representations associated to these cuspidal eigenforms, and in the case of weight two, these representations are somewhat concretely obtained as representations occurring in the natural action of Galois on the $p$-power torsion points of abelian varieties.

In particular, in our discussion of $p=691$, we chose to single out $\Delta$, the eigenform of weight twelve (which we will also call $\Phi_{12}$ in Section 13 below) but Ken worked with weight two, and in that specific instance he would be visualizing the Galois representation obtained by the natural Galois action on the 691-power torsion points of the abelian variety of dimension 2508 that we called $A$, attached to the eigenform we will be calling $\Phi_{2}$ in Section 13 .

- $5 \Rightarrow 4$.

Let $f=\sum_{n=1}^{\infty} a_{n}(f) q^{n}$ be a cuspidal newform of level $p$ (i.e., on $\Gamma_{1}(p)$ ) of weight $>1$. Deligne's Theorem (see Section 9 below) provides us with degree two Galois representations as discussed previously in the context of 
$\Delta$. In particular, if we view the Fourier coefficients of $f$ as lying in some finite discrete valuation ring extension $D$ of $\mathbf{Z}_{p}$ (this generally requires making a choice), then Deligne will give us a representation $\rho_{D}: G_{\mathbf{Q}} \rightarrow$ $\mathrm{GL}_{2}(D)$ which is

- irreducible as a representation over the field of fractions of $D$, and

- which has the property that the trace of Frobenius at $\ell$ (for primes $\ell \neq p)$ is equal to $a_{\ell}(f)$.

For $f$ ranging through the package of eigenforms given to us by $\mathbf{5}$,

- if $D$ is the discrete valuation ring extension of $\mathbf{Z}_{p}$ generated by the Fourier coefficients of $f$, the residue field of $D$ is $\mathbf{F}_{p}$;

- the Fourier expansion of $f$ modulo the maximal ideal of $D$ is congruent to the Fourier expansion of $E_{2 k}$ modulo $p$ making

- the Galois representation $\bar{\rho}_{D}$ (i.e., $\rho_{D}$ viewed over $\mathbf{F}_{p}$ ) be reducible; with, in fact,

- its semisimplification $\bar{\rho}_{D}^{\text {ss }}$ being equal to the trivial character 1 plus $\omega^{2 k-1}$, where $\omega$ is the cyclotomic character modulo $p$, i.e., $\omega$ is the composition

$$
G_{\mathbf{Q}} \rightarrow \operatorname{Gal}\left(\mathbf{Q}\left(e^{2 \pi i / p}\right) / \mathbf{Q}\right) \stackrel{\iota}{\simeq} \mathbf{F}_{p}^{*}
$$

The collection of eigenforms $f$ given to us by $\mathbf{5}$ have further properties making them quite a coherent package; all this will play a role in the later parts of this article. For example, the $p$ th Fourier coefficient, $a_{p}(f)$ (which is also the eigenvalue of $U_{p}$, the Atkin-Lehner automorphism acting on $f$ ) is congruent to 1 modulo the maximal ideal of $D$, which implies that $f$ is what is called p-ordinary, which has strong implications regarding the restriction of the associated Galois representation to an inertia group at $p$ (cf. Definition 10 in Section 201). Also, there is some uniformity in the nature of the discrete valuation ring $D$, in that one can take - once and for all $f$ 's in the package $-D$ to be a fixed finite flat extension of $\mathbf{Z}_{p}$.

If we choose, as Ken did, to work with one of the eigenforms $f$ as above of weight two, then $f$ cuts out in a standard manner a simple abelian subvariety $A=A_{f} \subset J_{1}(p)$ - unique up to $\mathbf{Q}$-isogeny-having all the propertie:24 described in 4 .

- $4 \Rightarrow 3$.

So far, this seems like a very unpromising picture, for we have no idea whether the representation $\bar{\rho}_{D}$ is nothing more than the sum $\mathbf{1} \oplus \omega^{2 k-1}$, which would be a nice-enough fact, but would not give us what we want, namely a nontrivial abelian Galois extension of the $p$-cyclotomic field (and, in fact, we even want more: we want it to be everywhere unramified and acted upon by Galois in a very particular way).

It is at this point that the Ribet wrench comes to help us. Ken uses it to show that since the representation over $F$, the field of fractions of $D$, is irreducible, he can change the $D$-lattice of the underlying $F$-vector space of the representation $\rho_{D} \otimes F$ to obtain a new $G_{\mathbf{Q}}$-stable $D$-lattice such that the corresponding $\mathbf{F}_{p}$-representation obtained by reduction modulo

\footnotetext{
${ }^{24}$ Consider, in the appropriate Hecke algebra, the ideal $I_{f}$ of elements annihilating the modular form $f$; then $A_{f}$ is an abelian variety that is $\mathbf{Q}$-isogenous to the quotient abelian variety $J_{1}(p) / I_{f}$. $J_{1}(p)$. This construction goes back to Shimura.
} 
the maximal ideal is indecomposable and in fact, in this situation, one can do this in two ways: either so as to have the trivial representation $\mathbf{1}$ as subrepresentation of the corresponding indecomposable residual representation or as quotient. Ken goes with quotient and uses the algebraic geometry of the abelian variety $A$ to guarantee that he has constructed precisely the type of abelian extension that is required by $\mathbf{3}$. We call this the preferred indecomposable residual representation. We shall be going into a few details about this last point in Section 18 below.

- $3 \Rightarrow \mathbf{2}$.

This leg of the journey is guaranteed by Class Field Theory as discussed briefly in Subsection 4.1 above.

- $2 \Rightarrow 1$.

This is Herbrand's theorem (proved before World War II) 25

I hope that my article has, up to this point, conveyed to readers of general background the flavor of some ideas behind this type of explicit construction of abelian extensions. The remaining sections of this article will go a bit further into some of the techniques required for, and connected to, the proof, and also current related work.

\section{PART II: \\ Galois Representations Coming from Algebraic Geometry}

\section{Galois EXtensions of NUMBer Fields, PRIME SPLITTING PHENOMEnA, AND Frobenius CONJUGACY CLASSES}

Let $L / K$ be a finite Galois extension of number fields, and put $G=\operatorname{Gal}(L / K)$. The action of the group $G$ on $L$ stabilizes the ring of integers $\mathcal{O}_{L}$ in $L$ and fixes the subring $\mathcal{O}_{K}=\mathcal{O}_{L} \cap K$ of integers in $K$. Of major arithmetic interest is the manner in which primes $P$ of $K$ (i.e., nonzero prime ideals of $\mathcal{O}_{K}$ ) split (or not) in $L$. A historically important case of this general problem is answered by the theorem ascribed to Fermat that says that a prime number is expressible as a sum of two squares (i.e., splits into a product of two primes in the field of Gaussian numbers) if and only if it is not congruent to $-1 \bmod 4$. In general, the splitting of $P$ in the field $L$ is given by the prime factorization of the $\mathcal{O}_{L}$-ideal $P \cdot \mathcal{O}_{L}$. Such a factorization will look like

$$
P \cdot \mathcal{O}_{L}=Q_{1}^{e} \cdot Q_{2}^{e} \cdot \ldots \cdot Q_{m}^{e},
$$

where the $Q_{i}$ are mutually distinct primes of $L$ and $e \geq 1$.

One usually refers to the primes $Q_{i}$ that occur in this formula as the "primes $Q$ of $L$ lying above $P$ ".

For any prime $P$ of $K$ the action of the Galois group $G$ on $\mathcal{O}_{L}$ induces a transitive action on the set of primes of $L$ lying above $P$. For any of these $Q$ 's, $G_{Q} \subset G$ denotes the isotropy subgroup 26 at $Q$ of the action, i.e., $G_{Q}:=\{g \in G \mid g \cdot Q=Q\}$. Fixing, then, a prime $Q$ lying above $P$ we may identify the set of all primes of $L$ lying above $P$ with the left coset space $G / G_{Q}$ in the evident way. The isotropy subgroup $G_{Q}$ stabilizes both $\mathcal{O}_{L}$ and $Q \subset \mathcal{O}_{L}$, and therefore one induces a natural action of $G_{Q}$ on the finite ("residue field at $Q$ ") $\kappa_{Q}:=\mathcal{O}_{L} / Q$.

\footnotetext{
25 [26]; see also 46].

${ }^{26}$ This is also called the decomposition group.
} 
The prime $P$ is said to be ramified in $L / K$ if $e>1$; there are only finitely many primes $P$ ramified in $L / K$ (these being the primes dividing the discriminant $\operatorname{Disc}(L / K)$ which is a nonzero ideal of $\left.\mathcal{O}_{K}\right)$. If, for any nonzero ideal $I \in \mathcal{O}_{K}$, we denote by $N I$ its norm (meaning the cardinality of the set $\mathcal{O}_{K} / I$ ), then a sufficient condition for $P$ to be unramified is that the integer $N P$ not divide $N \operatorname{Disc}(L / K)$.

In the case where $P$ is unramified in $L / K$, the isotropy group $G_{Q}$ for any $Q$ lying above $P$ has a strikingly precise structure:

The isotropy group $G_{Q}$ is cyclic with a canonical generator (called the Frobenius element at $Q$ )

$$
\operatorname{Frob}_{Q} \in G_{Q} \subset G
$$

determined uniquely by the property that the natural action of Frob $_{Q}$ on the residue field at $Q, \mathcal{O}_{L} / Q$, consists in raising every element to the NPth power; equivalently,

$$
\operatorname{Frob}_{Q}(x) \equiv x^{N P} \bmod Q \quad \text { for } x \in \mathcal{O}_{L} \text {. }
$$

The set of elements Frob $_{Q} \in G$, where $Q$ ranges through all primes of $L$ lying above $P$, consists in a single conjugacy class of $G$ (determined by $P$ ) and we will refer to this conjugacy class $c_{P}=c_{P}(L / K) \subset \operatorname{Gal}(L / K)$ as the Frobenius conjugacy class (relative to the Galois extension $L / K$ ) attached to the (unramified) prime $P$ of $K$. This assignment is nicely functorial for nested Galois extensions of $K$; i.e., if $K \subset L \subset M$ are fields with $M / K$ and $L / K$ Galois, we have, for all primes $P$ of $K$ unramified in $M / K$, that $c_{P}(L / K)$ is the image of $c_{P}(M / K)$ under the natural surjection $\operatorname{Gal}(M / K) \rightarrow \operatorname{Gal}(L / K)$.

The Galois group $G=\operatorname{Gal}(L / K)$ of a Galois extension $L / K$ of number fields comes, then, with an impressive amount of extra structure. There are many ways of bottling this extra structure but here is a way that I find helpful.

By a Cebotarev group over a number field $K$ I will mean a finite group $G$ together with a function $P \mapsto c_{P}$ defined on almost all (i.e., all but a finite number of) primes $P$ over $K$ that associates to $P$ a conjugacy class $c_{P}$ in $G$, and that has the property that for any conjugacy class $c \subset G$, there are infinitely many $P$ with $c_{P}=c$ and more precisely:

$$
\lim _{X \rightarrow \infty} \frac{\mid\left\{P \mid c_{P}=c \text { and } N P<X\right\} \mid}{|\{P \mid N P<X\}|}=\frac{|c|}{|G|}
$$

where the absolute value sign around the symbol for a set means its cardinality.

Let us say that two Cebotarev groups over $K,\left\{G ; P \mapsto c_{P}\right\}$ and $\left\{G^{\prime} ; P \mapsto c_{P}^{\prime}\right\}$, are isomorphic if there is an isomorphism $G \simeq G^{\prime}$ which sends $c_{P}$ to $c_{P}^{\prime}$ for all but finitely many primes $P$ for which both $c_{P}$ and $c_{P}^{\prime}$ are defined.

We can formulate a version of the Cebotarev Theorem as follows:

Theorem 2. Let $L / K$ be a Galois extension of number fields with $G=\operatorname{Gal}(L / K)$. The rule that assigns to each prime $P$ of $K$ that is unramified in $L / K$ the Frobenius conjugacy class $c_{P}=c_{P}(L / K)$ defines a Cebotarev group $\left\{G ; P \mapsto c_{P}\right\}$. The isomorphism class of this Cebotarev group over $K$ determines $L / K$ up to isomorphism. 
The first sentence of the theorem is the classical Cebotarev Theorem 27 The second assertion is seen as follows. Let $\bar{K} / K$ be an algebraic closure of $K$.

Lemma 1. Let $L, L^{\prime} \subset \bar{K}$ be subfields, each of which is a finite Galois extension field of $K$, and suppose that there is an isomorphism $\psi: \operatorname{Gal}(L / K) \simeq \operatorname{Gal}\left(L^{\prime} / K\right)$ such that if $P$ runs through almost all primes of $K$ which are unramified in $L / K$ and in $L^{\prime} / K$, the isomorphism $\psi$ sends the Frobenius conjugacy class relative to the Galois extension $L / K, c_{P} \subset G=\operatorname{Gal}(L / K)$, to the Frobenius conjugacy class relative to the Galois extension $L^{\prime} / K, c_{P}^{\prime} \subset G^{\prime}=\operatorname{Gal}\left(L^{\prime} / K\right)$. Then $L^{\prime}=L$.

Proof. Let $M=L \cdot L^{\prime} \subset \bar{K}$ be the compositum. Consider the injection

$$
\operatorname{Gal}(M / K) \hookrightarrow G \times G^{\prime}
$$

defined as the product of the natural surjections onto each factor.

Suppose that the natural surjection $\operatorname{Gal}(M / K) \rightarrow G$ is not an isomorphism.

Let $\mathcal{C}^{M / K} \subset \operatorname{Gal}(M / K)$ denote the conjugacy class in $\operatorname{Gal}(M / K)$ containing some nontrivial element of $N:=\operatorname{ker}\{\operatorname{Gal}(M / K) \rightarrow G\}$. The natural projection $N \rightarrow G^{\prime}$ is injective since $\operatorname{Gal}(M / K) \hookrightarrow G \times G^{\prime}$ is injective. Let $\mathcal{C}^{\prime} \subset G^{\prime}$ be the conjugacy class of $G^{\prime}$ that is the image of $\mathcal{C}^{M / K}$ under the natural surjection $\operatorname{Gal}(M / K) \rightarrow G^{\prime}$, noting that $\mathcal{C}^{\prime}$ is not the (conjugacy class of the) identity in $G^{\prime}$. In contrast, if $\mathcal{C}$ denotes the image of $\mathcal{C}^{M / K}$ under the natural surjection $\operatorname{Gal}(M / K) \rightarrow G$, then $\mathcal{C}=\{1\}$ is the conjugacy class of the identity in $G$.

Let $P$ be a prime of $K$ so that

(1) the conjugacy class $\mathcal{C}^{M / K} \subset \operatorname{Gal}(M / K)$ is the conjugacy class of Frobenius elements for the prime $P$ relative to the extension $M / K$, and

(2) the conjugacy classes $c_{P} \subset G$ and $c_{P}^{\prime} \subset G^{\prime}$ are both defined (i.e., by the Cebotarev group structures of each of these groups).

This is possible since by the Cebotarev Theorem for $M / K$ every conjugacy class of $\operatorname{Gal}(M / K)$ is the Frobenius conjugacy class of infinitely many primes of $K$.

By the functoriality of Frobenius conjugacy classes mentioned above, we have that $\mathcal{C}=c_{P}$ is the conjugacy class of Frobenius elements for the prime $P$ relative to the extension $L / K$. Similarly, $\mathcal{C}^{\prime}=c_{P}^{\prime}$ is the conjugacy class of Frobenius elements for the prime $P$ relative to the extension $L^{\prime} / K$. By the hypotheses of our lemma, we would then have that $\psi(\mathcal{C})=\mathcal{C}^{\prime}$. But $\mathcal{C}$ is the identity conjugacy class in $G$ and $\mathcal{C}^{\prime}$ is not the identity class in $G^{\prime}$. Since $\psi$ is an isomorphism of groups, we have a contradiction.

It follows that $N$ is the trivial group, and therefore the natural projection $\operatorname{Gal}(M / K) \rightarrow G$ is an isomorphism, establishing our lemma.

It is traditional to study the structure packaged in the Cebotarev group associated to an extension $L / K$ by considering linear representations of the underlying Galois group, i.e., by choosing a Galois representation over $K$ that is split by $L / K$ and using the vocabulary and techniques of analytic number theory 28

Here is a brief hint of how one passes to analytic number-theoretic methods. Fix $\left\{G ; P \rightarrow c_{P}\right\}$, a Cebotarev group over a number field $K$. Consider an (irreducible)

\footnotetext{
${ }^{27}$ For a proof of the theorem, cf. Thm. 10, Ch. VIII, Section 4 of [39]; also see Section 2.7 of 53, which casts the theorem in the context of global fields. For Cebotarev's original article, see [13, and to get a sense of his generosity of spirit, see the extraordinary tale beginning on page 131 in [25].

${ }^{28} \mathrm{In}$ fact, it is by such a route that the Cebotarev Theorem quoted above is proved.
} 
complex representation $\eta: G \rightarrow \mathrm{GL}_{n}(\mathbf{C})$. For a prime number $P$ for which $c_{P}$ is defined, let

$$
\mathcal{L}_{\eta, P}(T):=\operatorname{det}\left(1-T \cdot M_{P}\right) \in \mathbf{C}[T]
$$

be the characteristic polynomial of the $n \times n$ matrix $1-T \cdot M_{P} \in \operatorname{Mat}_{n \times n}(\mathbf{C}[T])$, where $M_{P} \in \mathrm{GL}_{n}(\mathbf{C})$ is any element of $\eta\left(c_{P}\right) \subset \mathrm{GL}_{n}(\mathbf{C})$. Thus,

$$
\mathcal{L}_{\eta, P}(T) \equiv 1-\operatorname{Trace}\left(\eta\left(c_{P}\right)\right) \cdot T \text { modulo higher powers of } T \text {. }
$$

Since $\eta$ is a complex representation of a finite group, and since the Cebotarev Theorem guarantees that every conjugacy class of $G$ is $c_{P}$ for (infinitely many) primes $P$, the representation $\eta$ is already pinned down, up to isomorphism, by the data $P \mapsto \operatorname{Trace}\left(\eta\left(c_{P}\right)\right)$ and therefore all the more by

$$
P \mapsto \mathcal{L}_{\eta, P}(T) .
$$

From the above data, define a Dirichlet series (in the complex variable $s$ ) by

$$
\mathcal{L}(\eta, s)=\prod_{P} \mathcal{L}_{\eta, P}\left(N P^{-s}\right)^{-1},
$$

where the product is taken over all those primes $P$ for which the Cebotarev group structure provides us with a " $c_{P}$ ". Since the eigenvalues of any of the matrices $M_{P}$ in the previous paragraph are roots of unity, a straightforward computation gives that the Dirichlet series $\mathcal{L}(\eta, s)$ converges in some right half-plane. One learns detailed statistical information about the rule $P \mapsto c_{P}$ by establishing further analytic properties (e.g., analytic continuation, functional equation, location of poles and zeroes) of this collection of Dirichlet series

$$
\eta \mapsto \mathcal{L}(\eta, s)
$$

for various representations $\eta$.

If the Cebotarev group in question comes from a Galois extension $L / K$, we may view $\eta$ as a representation of $\operatorname{Gal}(L / K)$, and $\mathcal{L}(\eta, s)$ is, except for factors corresponding to the (finitely many) missing primes $P$, the Artin $L$-function about which much is known, and even more is conjectured.

\section{TOWers of Galois Representations; $p$-ADiC Galois REPRESEnTATIONS}

In the previous section we discussed finite degree Galois extensions of number fields and corresponding Galois representations of finite Galois groups into $\mathrm{GL}_{n}(\mathbf{C})$. The focus, though, of much recent work is towards infinite degree extensions. For this we should say a few words about infinite Galois groups.

For $K$ a field, choose a separable algebraic closure $K^{\text {sep }}$ of $K$ and set $G_{K}:=$ $\operatorname{Gal}\left(K^{\mathrm{sep}} / K\right)$, which we view as a profinite topological group with its Krull topology; this is the topology for which closed subgroups of $G_{K}$ are in one-to-one correspondence - as they would be in Galois theory of finite degree extensionswith the intermediate subfields of $K^{\text {sep }} / K$; any such closed subgroup $H \subset G_{K}$ corresponds to the subfield consisting of the elements of $K^{\text {sep }}$ fixed by all the elements of $H$. Since $G_{K}$ is a profinite group, its continuous representations to Lie groups over $\mathbf{R}$ or over $\mathbf{C}$ necessarily factor through finite quotient groups, but this is no longer true if the target groups are, for example, Lie groups over $p$-adic fields. 
By a p-adic Galois representation of degree $n$ over $K$ we will mean a continuous representation

$$
G_{K} \longrightarrow \mathrm{GL}_{n}(F)
$$

where $F$ is some extension field of finite degree over $\mathbf{Q}_{p}$, the field of $p$-adic numbers. Since $G_{K}$ is compact, one can show that any such continuous homomorphism can be conjugated to one that factors through $\mathrm{GL}_{n}\left(\mathcal{O}_{F}\right) \subset \mathrm{GL}_{n}(F)$, where $\mathcal{O}_{F}$ is the ring of integers (i.e., elements integral over $\mathbf{Z}_{p}$ ) in $F$. That is, in any equivalence class of such representations, there will be at least one homomorphism that has its image in $\mathrm{GL}_{n}\left(\mathcal{O}_{F}\right)$. For example, if $F=\mathbf{Q}_{p}$, such a representation factors through a homomorphism

$$
G_{K} \longrightarrow \mathrm{GL}_{n}\left(\mathbf{Z}_{p}\right)
$$

which itself can be viewed as a projective limit of homomorphisms,

$$
G_{K} \longrightarrow \mathrm{GL}_{n}\left(\mathbf{Z} / p^{\nu} \mathbf{Z}\right)
$$

for $\nu=1,2,3, \ldots$, these representations being split by finite Galois extensions of $K$,

$$
K \subset L_{1} \subset L_{2} \subset \cdots \subset L_{\nu} \subset \cdots,
$$

and if $L_{\infty}:=\bigcup_{\nu} L_{\nu}$, then $L_{\infty} / K$ is a Galois extension of $K$ whose (possibly infinite) Galois group is the compact $p$-adic Lie subgroup of $\mathrm{GL}_{n}\left(\mathbf{Z}_{p}\right)$ that is the image of $G_{K}$ in $\mathrm{GL}_{n}\left(\mathbf{Z}_{p}\right)$.

Often we will be content to deal with algebraic extension fields of $\mathbf{Q}_{p}$, i.e., subfields $F$ of $\overline{\mathbf{Q}}_{p}$, an algebraic closure of $\mathbf{Q}_{p}$, but sometimes it is useful to allow a certain larger field as field of scalars, namely the field $\mathbf{C}_{p}:=\hat{\overline{\mathbf{Q}}}_{p}$, the hat ${ }^{\wedge}$ signifying the completion of $\overline{\mathbf{Q}}_{p}$ with respect to its $p$-adic valuation 29

Usually we will be dealing with Galois representations that are unramified except possibly at a finite number of places. For such a Galois representation $\rho: G_{K} \longrightarrow$ $\mathrm{GL}_{n}(F)$ we have a convenient "numerical handle" that determines $\rho$, up to semisimplification, namely, the function that associates to each place $v$ of $K$ unramified in $\rho$ the value $a_{\rho}(v):=\operatorname{Trace}_{F}\left(\rho\left(\operatorname{Frob}_{v}\right)\right) \in F$. This function

$$
v \mapsto a_{\rho}(v)
$$

plays a central role in any dealings with a Galois representation $\rho$.

In particular, if $K=\mathbf{Q}$, we may view this function as taking values on "almost all" prime numbers $\ell$, i.e., $\ell \mapsto a_{\rho}(\ell)$ and note that we have already had hints of such functions, such as the Ramanujan function $\ell \mapsto \tau(\ell)$, alluded to in our discussion of 5 above. An excellent general introduction to $\ell$-adic representations is given in Serre's article [57] as well as in his earlier treatise [54].

\section{Deligne's Theorem for the MOdular Form $\Delta$}

A theorem of Deligne - a special case of which we shall be quoting below - gives us that for every prime number $p$ and every modular eigenform, there is a continuous irreducible degree two $p$-adic Galois representation that is closely related to the eigenform in the sense that the Fourier coefficients of the eigenform determine, in a fairly direct way, the equivalence class of the representation. The previous two

\footnotetext{
${ }^{29}$ This field $\mathbf{C}_{p}$ is sometimes called "Tate's $p$-adic complex numbers" the Tate part of its name because Tate first defined and used it, the complex numbers part of its name because $\mathbf{C}_{p}$ is (deprived of its topology) abstractly isomorphic to the classical field of complex numbers; $\mathbf{C}_{p}$ is, in particular, algebraically closed, and (being a completion) is complete.
} 
sections give us the vocabulary we need to discuss such connection between modular forms such as

$$
\Delta(q)=q \prod_{n=1}^{\infty}\left(1-q^{n}\right)^{24}=\sum_{n=1}^{\infty} \tau(n) q^{n}
$$

and the $p$-adic Galois representations that connect to them.

In this section, to focus ideas, we will concentrate on the modular form $\Delta$ itself, and in the next section we will specialize even further by considering $p=691$. Our modular form $\Delta$ is related by the mod 691 congruence to the Eisenstein series $E_{12}$ as we discussed in Part I. We will be introducing a representation denoted $\rho_{\Delta, 691}$ that will be the key for us in constructing the abelian field $L^{\{12\}}$ and the Galois representation $\rho^{\{12\}}$ that cuts it out. A very similar discussion beginning with the 691-adic cuspform $\Delta^{\{200\}}$ would construct the abelian field $L^{\{200\}}$ and the Galois representation $\rho^{\{200\}}$ that cuts it out (see footnote (19) above).

The essential input here is the theorem of Deligne relating modular eigenforms to Galois representations:

Theorem 3 (Deligne). Let $p$ be a prime number. There is a continuous irreducible degree two Galois representation

$$
\rho_{\Delta, p}: G_{\mathbf{Q}} \rightarrow \operatorname{Aut}_{\mathbf{Q}_{p}}(V) \approx \mathrm{GL}_{2}\left(\mathbf{Q}_{p}\right)
$$

(where $V$ is a two-dimensional $\mathbf{Q}_{p}$-vector space) such that for all primes $\ell \neq p$ the representation $\rho_{\Delta, p}$ is unramified at $\ell$ and has the property that the trace of Frobenius at $\ell$ is equal to $\tau(\ell) \in \mathbf{Z} \subset \mathbf{Q}_{p}$.

Proof. See [19].

This condition (that the trace of Frobenius at $\ell$ is equal to $\tau(\ell) \in \mathbf{Z} \subset \mathbf{Q}_{p}$ for all primes $\ell \neq p$ ) determines the character of $\rho_{\Delta, p}$ since the Frobenius elements are dense, and therefore (since $\rho_{\Delta, p}$ is irreducible) the representation is pinned down if we know the Fourier coefficients of $\Delta$. In the discussion to follow, first let us suppose that $p$ is any (odd) prime number.

Suppose you are given a $G_{\mathbf{Q}^{-}}$-stable lattice $M \subset V$ (so $M$ is a free $\mathbf{Z}_{p}$-module of rank two). By passing to $\bar{V}=M / p M=M \otimes \mathbf{z}_{p} \mathbf{F}_{p}$ we get an $\mathbf{F}_{p}$-representation of $G_{\mathbf{Q}}$ of degree two,

$$
\bar{\rho}_{\Delta, p, M}: G_{\mathbf{Q}} \rightarrow \operatorname{Aut}_{\mathbf{F}_{p}}(\bar{V}) \approx \mathrm{GL}_{2}\left(\mathbf{F}_{p}\right),
$$

that may - and in the cases of specific interest to us, will - depend upon the choice of the lattice $M$. We will refer to $\bar{\rho}_{\Delta, p, M}$ as the residual representation obtained from $\rho_{\Delta, p}$ via the lattice $M$. Since (by the Cebotarev Density Theorem) the conjugacy classes of Frobenius elements (associated to all prime numbers $\ell \neq p$ ) run through all conjugacy classes of the Galois group of the splitting field of $\bar{\rho}_{\Delta, p, M}$, and, since $p$ is odd, the character of the (degree two) representation $\bmod p, \bar{\rho}_{\Delta, p, M}$, determines its semisimplification, we get that this semisimplification is completely characterized 30 by the function

$$
\ell \mapsto \tau(\ell) \quad \bmod p .
$$

For general prime numbers $p$ the beauty of the representation $\rho_{\Delta, p}$ is that it is obtained naturally, and systematically, from the evident action of Galois on a piece of the étale cohomology group of an algebraic variety. The mild difficulty one

\footnotetext{
${ }^{30}$ See 18 and the proof, especially page 216 of (30.16), i.e., the Brauer-Nesbitt Theorem.
} 
encounters in working with this is that we are dealing with an $H^{11}$, i.e., cohomology in dimension eleven.

\section{Galois Representations}

\section{ATTACHED TO MORE GENERAL MODULAR EIGENFORMS}

For a fine introduction to the subject of the title of this section, see Ribet's paper [50]. Passing from our modular form $\Delta$ to a more general context, let $N \geq 1$ and consider modular cuspidal eigenforms of any weight $w \geq 1$ for $\Gamma_{0}(N)$ with nebentypus. Let $\Phi$ be such an eigenform, with Fourier expansion given as

$$
\Phi=0+q+\sum_{n=2}^{\infty} t(n) q^{n}
$$

with coefficients $t(n)$ all lying in the ring of integers $O$ of some number field. For any prime $p$ and prime ideal $\lambda \subset O$ lying over $p$, let $O_{\lambda}$ denote the completion of $O$ at $\lambda$, so that $O_{\lambda}$ is a discrete valuation ring, finite flat over $\mathbf{Z}_{p}$.

Theorem 4. There is a unique irreducible Galois representation

$$
\rho_{\Phi, \lambda}: G_{\mathbf{Q}} \rightarrow \mathrm{GL}_{2}\left(O_{\lambda}\right)
$$

unramified outside $p \cdot N$ and such that for all primes $\ell$ not dividing $p \cdot N$, we have that if Frob $_{\ell} \in G_{\mathbf{Q}}$ is a choice of Frobenius element at $\ell$, the trace of

$$
\rho_{\Phi, \lambda}\left(\operatorname{Frob}_{\ell}\right) \in \mathrm{GL}_{2}\left(O_{\lambda}\right)
$$

is equal to the image of the Fourier coefficient $t_{\ell} \in O$ in $\mathcal{O}_{\lambda}$.

The determinant, $\operatorname{det}\left(\rho_{\Phi, \lambda}\right)$, of this representation is the character whose values at any Frobenius element Frob $_{\ell}$ for $\ell$ any prime not dividing $p \cdot N$ is equal to $\epsilon(\ell) \ell^{w-1}$, where $\epsilon$ is the nebentypus character associated to $\Phi$.

This theorem was first proved for weight $w=2$ by Shimura (cf. [61, Chapter 7; [60]). The case $w \geq 2$ was conjectured by Serre and proved by Deligne (cf. [19]); the case $w=1$ was subsequently done by Deligne and Serre ([21]).

\section{Moving From One Weight to ANOther}

The way in which Deligne and Serre proved their theorem (21]), cited at the end of the previous section, was to first move from weight one to higher weight by multiplying by an appropriate Eisenstein series to obtain a modular form, no longer necessarily a Hecke eigenform, but with Fourier coefficients connected by congruences to the Fourier coefficients of the initial eigenform of weight one. An appropriate "spectral decomposition" of that modular form under the action of the Hecke algebra then provided them with eigenforms for which one could apply the theorem of Deligne 31

The above operations had the effect of replacing an eigenform $f$ of one weight by an eigenform $g$ of a higher weight that is related to $f$ by a congruence mod $p$ between their corresponding Fourier coefficients. In more recent times, one has

\footnotetext{
${ }^{31}$ Alternatively, as Shimura mentioned to Serre (see the footnote on page 312 of [56] and its reference to [38), multiplying by an approprate Eisenstein series of weight one would allow one to apply the more elementary prior result of Shimura.
} 
somewhat greater flexibility, in that, for example, the theory of Hida 32 provides a clean way of starting with a cuspidal eigenform $f$ of weight $w_{o}$ and level $N$ that is $p$-ordinary $(p \geq 5)$ and producing from $f$ a fairly nicely behaved collection of ( $p$-ordinary) eigenforms $f_{w}$ for every integral weight $w \geq 2$ such that $f_{w_{o}}=f$ and such that the Fourier coefficients of all of these eigenforms are related by a congruence 33

In particular, if you are principally interested in the properties of your eigenform modulo $p$ you can replace it by one of weight two, and therefore find its associated Galois representation realized as the Galois module of one-dimensional cohomology with coefficients in $\mathbf{Q}_{p}$ of some simple abelian variety; or, to put it even more concretely, realized in the action of Galois on the $p$-torsion points of such an abelian variety 34 Since the weight two modular form has Fourier coefficients congruent modulo $p$ to the high weight eigenform we started out with, the $\bmod p$ Galois representations associated to these modular forms will be equivalent, at least after semisimplification, as discussed in Part I (Subsection 4.5).

Moving in this way to weight two modular eigenforms and their associated abelian varieties, we have, at least, the sensation that we are dealing with more concrete entities (than higher-dimensional varieties and the Galois representations on their étale cohomology groups) and indeed, in certain instances, working with modular forms of weight two and their associated abelian varieties actually does confer the advantage of significantly more control.

\section{Returning TO $p=691$}

Here is what happens in the special case $p=691$ : since as we have mentioned,

$$
\tau(\ell) \equiv 1+\ell^{11} \bmod 691
$$

for every prime number $\ell$, we get that the semisimplification of $\bar{\rho}_{\Delta, 691, M}$ is equivalent to the semisimplification of the representation $\bar{\rho}$ of $\mathbf{4}$, namely, to $\mathbf{1} \oplus \omega^{11}$. This is true for the representations $\bar{V}=M / p M$ obtained from every $G_{\mathbf{Q}^{-}}$-stable lattice $M \subset V$. In the 1967/1968 Séminaire Delange-Pisot-Poitou, Serre wrote that it seemed probable to him that the image of inertia at $p$ under the representations $\bar{\rho}_{\Delta, 691, M}$ was distinct from the image of the full Galois group, thereby generating an everywhere unramified cyclic extension of $\mathbf{Q}\left(e^{2 \pi i / 691}\right)$ of degree 691 . Serre went on to suggest that, perhaps, one could determine this using an analysis of the representation attached to $\Delta$ modulo $691^{2}$ (page 507 of [55]).

This was the approach similar to one taken up later by Ralph Greenberg and Paul Monsky who indeed constructed the sought-for everywhere unramified cyclic extension. This is unpublished. Using the fact that $p=691$ is properly irregular (and therefore the converse to Herbrand's Theorem is known for $p=691$ ) and also

\footnotetext{
${ }^{32}$ Here are a few basic references to Hida's theory, but see also his web-page (http://www.math.ucla.edu/ hida/). One of the main ingredients in the theory that we are using is his Control Theorem. For a complete proof of this over a general totally real field, see Theorem 3.2 of [29]; for a general discussion of the theory based on notes of a year-long course that Hida gave, taking into account Wiles' theory (specifically, the universality of the Hecke algebra in the ordinary case), see [30] (Sections 2,3; and Theorem 1 especially); for a further perspective, see [27, 28].

${ }^{33}$ If, for example, the starter eigenform $f=f_{w_{o}}$ is on $\Gamma_{0}(N)$, then all the eigenforms $f_{w}$ will be on $\Gamma_{0}(N) \cap \Gamma_{1}(p)$.

${ }^{34}$ There will, in general, be more than one such simple abelian variety, but (at least) one of them will be a quotient of $J_{1}(p) / J_{0}(p)$; here we have used standard notation.
} 
using facts about the representation attached to $\Delta$ modulo $691^{2}$ that guarantee Proposition 1 below, Greenberg and Monsky showed that the piece of the 691Hilbert class field of $\mathbf{Q}\left(e^{2 \pi i / 691}\right)$ corresponding to the character $\omega^{-11}$ is contained in the field extension of $\mathbf{Q}$ that we called $L^{\{12\}}$ in Part I) cut out by Deligne's 691adic representation of $G_{\mathbf{Q}}$ associated with $\Delta$. Greenberg wrote to me: "I was quite excited by the idea behind this at the time because I thought that it suggested a very promising approach to the converse."

Proposition 1 (Monsky, Greenberg).35 Up to homothety (i.e., multiplication by a nonzero scalar) there are only two Galois stable lattices $M$ as above, and the action of the Galois group $G_{\mathbf{Q}}$ on $M / p M$ for each of these lattices is triangular and non-semisimple (or equivalently: reducible and indecomposable) with diagonal characters 1 and $\omega^{11}$ occurring in the two different orders in the two lattices.

13. Moving From $\Delta$ TO EIGENFOrms of Weight $2,3,4, \ldots($ When $p=691)$

In the previous paragraph we were considering any prime number $p$ and any eigenform. But if you fix attention to the weight 12 eigenform $\Delta$ and $p=691$ you are in quite a nice situation. Here, thanks to Hida's theory, there will be, for any weight $w>1$, a 691-adic cuspidal eigenform $\Phi_{w}=\Phi_{w, 691}$ of weight $w$ on $\Gamma_{1}(p)$ with Fourier coefficients in $\mathbf{Z}_{p}$ and whose Fourier expansion is of the form

$$
1 \cdot q^{1}+\sum_{n=2}^{\infty} a_{w}(n) q^{n},
$$

where the cofficients $a_{w}$ are algebraic numbers in $\mathbf{Q}_{691}$, and this Fourier series is congruent modulo 691 to the series

$$
0+\sum_{n=1}^{\infty}\left(\sum_{0<d \mid n} d^{11}\right) q^{n}
$$

modulo 691 ; i.e., $\Phi_{w}$ has the same Fourier expansion modulo 691 as $E_{12}$ or $\Delta$. (Indeed, $\Phi_{12}=\Delta$.)

If $\Phi_{w}$ is the 691-adic modular form of weight $w$ for $w=2,3,4, \ldots$ as above, denote by $\mathcal{F}_{w} \subset \mathbf{Q}_{691}$ the smallest field in $\mathbf{Q}_{691}$ generated (over $\mathbf{Q}$ ) by all the Fourier coefficients, $\left\{a_{w}(n) ; n=1,2, \ldots\right\}$, of $\Phi_{w}$. It is known that $\mathcal{F}_{w}$ is a "number field," i.e., is of finite degree over $\mathbf{Q}$.

Furthermore, in our case, the 691-adic cuspidal form $\Phi_{w}$ with the properties listed above is unique, for each $w>1$. Section 24 below sketches a proof of this. Deligne's Theorem provides us with an infinite sequence of 691-adic representations,

$$
\rho_{\{w, 691\}}: G_{\mathbf{Q}} \rightarrow \mathrm{GL}_{2}\left(\mathbf{Z}_{691}\right)
$$

(i.e., related to $\Phi_{w}$ for $w=2,3,4, \ldots$ ), all of them having the same semisimplication when reduced modulo 691. Specifically, its reduction $\bar{\rho}_{\{w, 691\}}: G_{\mathbf{Q}} \rightarrow \mathrm{GL}_{2}\left(\mathbf{F}_{691}\right)$ is a reducible representation and its semisimplication, $\bar{\rho}_{\{w, 691\}}^{\mathrm{ss}}$, is equivalent to $\mathbf{1} \oplus \omega^{11}$. These $\rho_{\{w, 691\}}$ all have the same preferred indecomposable residual representation,

\footnotetext{
${ }^{35}$ Greenberg tells me that one can check it just using the Hecke operators for the primes 2 and 3. Explicitly, since $\tau(2)=-24$ and $\tau(3)=252$ one must check that there is no integer $a$ simultaneously satisfying the following two congruences modulo $691^{2}=477481$ :

$$
-24 \equiv 2^{a}+2^{11-a} \text { and } 252 \equiv 3^{a}+3^{11-a} .
$$
}


as well, and this representation has the property that the inertia group at 691 acts semisimply.

William Stein and Craig Citro have calculated $\Phi_{2}$, and its field of Fourier coefficients $\mathcal{F}_{2}$, and I am thankful to them for providing me with the information I will be recounting here. Stein and Citro show that the (quotient) Hecke algebra, tensored with $\mathbf{Q}$, acting faithfully on the vector space of weight two cuspidal modular eigenforms of level 691 and nebentypus $\omega^{-10}$ is a field, and hence can be taken to be "our" $\mathcal{F}_{2}$. They show this field $\mathcal{F}_{2}$ to be of degree 57 over the cyclotomic field $\mathbf{Q}\left(\mu_{69}\right)$ (that's not a typo: $\omega^{-10}$ is of order 69 ). Moreover, there is a (unique) prime ideal $P$ of degree one with residual characteristic 691 in the ring of integers of $\mathcal{F}_{2}$ such that if $\mathcal{F}_{2, v}$ is the completion of the field $\mathcal{F}_{2}$ with respect to the valuation determined by $P$, then under the canonical identification $\mathbf{Q}_{691}=\mathcal{F}_{2, v}$, for every $n \geq 1$, the $n$th Hecke operator $T_{n} \in \mathcal{F}_{2} \subset \mathcal{F}_{2, v}=\mathbf{Q}_{691}$ is equal to $a_{2}(n) \in \mathbf{Q}_{691}$.

We are thus led to consider the abelian variety over $\mathbf{Q}$ that is simple (even over C) and related to the eigenform $\Phi_{2}$. Call this abelian variety $\tilde{A}$ for short (its standard name is $\left.J_{1}\left(691 ; \omega^{-10}\right)\right)$. Explicitly, $\tilde{A}$ is the abelian variety quotient of the Jacobian of the modular curve $J_{1}(691)$ associated to the space of weight two cuspidal modular eigenforms of level 691 and nebentypus $\omega^{-10}$. The calculations of Stein and Citro alluded to above give you that $\tilde{A}$ is an abelian variety of a whopping dimension $2508=2 \cdot 22 \cdot 57$, whose endomorphism $\operatorname{ring} \operatorname{End}_{\mathbf{C}}(\tilde{A})$ tensored with $\mathbf{Q}$ is equal to the field $\mathcal{F}_{2}$.

We know (see Corollary 5 and the first appendix, Section 24 below) that its Galois module of 691-power torsion points has a subquotient of length two that is equivalent to $\bar{\rho}$.

In other words, $\bar{\rho}$ can be "found in the action of Galois on 691-torsion points of $\tilde{A} . " \quad$ It is hard to believe that when we represent $\bar{\rho}$ in this manner we learn something 36 but we do 37

\section{PART III:}

\section{The WRENCH}

\section{VARIATIONS OF Galois REPRESENTATions}

It is natural nowadays (and for some problems imperative) to consider families of group representations that vary, in some sense or other "continuously" in terms of their parameters. Among those of specific interest to us here are families of $p$-adic representations of the Galois groups of fields, parametrized by complete local rings (and therefore called "Galois deformations", cf. [40]), or similar such families that vary $p$-adic analytically over $p$-adic analytic parameter spaces.

\footnotetext{
${ }^{36}$ We actually learn at least two things: The first being, as mentioned, that we have an elegant occurrence of the representation $\bar{\rho}$ in the context of abelian varieties. But the second is that when we return to the 691 -adic $G_{\mathbf{Q}}$-representation $\rho_{\Delta, 691}$ on the two-dimensional vector space $V$ over $\mathbf{Q}_{691}$, there is a unique choice of lattice, up to multiplication by a nontrivial scalar, for which $\bar{V}$ is a $G_{\mathbf{Q}}$-representation equivalent to $\bar{\rho}$ in 4 . The argument for this latter statement comes from Proposition 1 above and is also briefly discussed in Section 24

${ }^{37}$ To construct the abelian field $L^{\{200\}}$ (and the Galois representation $\rho^{\{200\}}$ that cuts it out) in a manner similar to the above, we would begin with the 691-adic cuspform $\Delta^{\{200\}}$ and would be discovering this Galois representation in Galois action on 691-power torsion in the abelian variety $J_{1}\left(691 ; \omega^{-198}\right)$. William Stein informs me that this is a simple abelian variety of dimension 4928 .
} 
In any of these contexts, suppose that you have such a continuous family of (Galois) group representations, the generic representation being irreducible. For argument's sake, suppose this family $\rho_{t}$ is parametrized by a line, with parameter variable denoted by $t$. For certain special values of $t$, say $t=t_{o}$, the representation $\rho_{t_{o}}$ may no longer be irreducible. Borrowing the standard logo that algebraic geometers use to depict degeneration in a parametrized family of objects, we may sometimes think of our family of representations as depicted in Figure 3 .

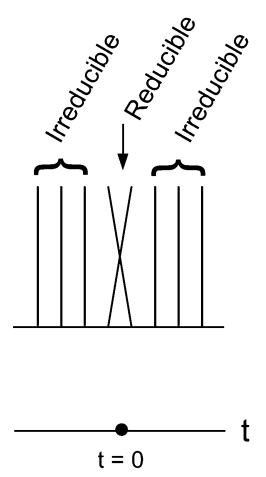

Figure 3

Note that absolute irreducibility for degree two representations is an "open condition" in the following sense: Let $R$ be an integral domain, $G$ a group, and consider $\rho: G \rightarrow \mathrm{GL}_{2}(R)$ a homomorphism viewed as a family of $G$-representations $\rho_{F}: G \rightarrow \mathrm{GL}_{2}(F)$ varying over the collection of homomorphisms $R \rightarrow F$ for fields $F$. If $\rho_{F_{o}}$ is reducible for any single injection $R \hookrightarrow F_{o}$, then for $R \rightarrow F$ any homomorphism of $R$ to any algebraically closed field $F, \rho_{F}$ is reducible. The contrapositive, of course, implies that if $\rho_{F}$ is absolutely irreducible for any single homomorphism $R \rightarrow F$, then for $R \hookrightarrow F_{o}$, any injection $\rho_{F_{o}}$ is absolutely irreducible.

Proof. Let $R \hookrightarrow F_{o}$ be an injection. Let $M$ be a free $R$-module with $G$-action via $\rho$. Suppose that $M \otimes_{R} F_{o}$ is not irreducible and that there exists a $G$-equivariant surjection $\phi: M \otimes_{R} F_{o} \rightarrow N$ to an $F_{o}$-vector space $N$ of dimension one endowed with $F_{o}$-linear $G$-action. The action of $G$ on $N$ factors through an abelian quotient of $G$ as does the action of $G$ on $\operatorname{ker} \phi$. Now consider the restriction of $\phi$ to $M$ and form

$$
0 \rightarrow M_{0} \rightarrow M \stackrel{\phi}{\rightarrow} M_{1} \rightarrow 0
$$

so that $M_{1} \subset N$ and $M_{0} \subset \operatorname{ker} \phi \subset M \otimes_{R} F_{o}$. In particular, the actions of $G$ on $M_{0}$ and $M_{1}$ factor through the abelian quotient of $G$. Now tensor with any field $F$ to get the $G$-equivariant exact sequence

$$
M_{0} \otimes_{R} F \rightarrow M \otimes_{R} F \stackrel{\phi}{\rightarrow} M_{1} \otimes_{R} F \rightarrow 0 .
$$

Since the actions of $G$ on the two flanking modules factor through the abelian quotient of $G$, it follows that either $M \otimes_{R} F$ is reducible as a degree two $G$-representation or else the action of $G$ on $M \otimes_{R} F$ factors through the abelian quotient of $G$. In either case, if $\bar{F}$ denotes an algebraic closure of $F$, we have that $M \otimes_{R} \bar{F}$ is reducible, and therefore $M \otimes_{R} F$ is not absolutely irreducible. 
The Jordan-Hölder constituents of $\rho_{t_{o}}$ are determined by the family $\left\{\rho_{t}\right\}_{t \neq t_{o}}$ parametrized by the complement of the point $t_{o}$. Nevertheless, as in algebraic geometric deformation theory (but not the less puzzling therefore) the isomorphism class of $\rho_{t}$ is not completely determined: there may be many distinct ways of filling in 38 the family of representations $\left\{\rho_{t}\right\}_{t}$ at $t_{o}$. Often there is one preferred indecomposable representation. This is the "lever" that can be used so effectively.

\section{A Lemma in the STYle of Ribet}

Ken's initial application was for $\kappa=\mathbf{Q}_{p}$ for some prime number $p$ and $\kappa=\mathbf{F}_{p}$.

For our discussion let $\mathcal{K}$ be a complete discrete-valued field with ring of integers $\mathcal{O}$, a choice of uniformizer $\pi$, and finite residue field (denoted $\kappa=\mathcal{O} / \pi \mathcal{O}$ ). As usual, $\mathcal{O}$ and $\mathcal{K}$ are given the $\pi$-adic topology, so that $\mathcal{O}$ is the projective limit of the finite discrete rings $\mathcal{O} / \pi^{n} \mathcal{O}$, and the $\mathcal{O}$-lattices $\pi^{-m} \mathcal{O} \in \mathcal{K}$ are open $\mathcal{O}$-submodules of $\mathcal{K}$, as is any $\mathcal{O}$-lattice in any finite-dimensional $\mathcal{K}$-vector space (given the standard topology).

Let $G$ be a profinite group and $V$ a $d$-dimensional $\mathcal{K}$-vector space endowed with a continuous $\mathcal{K}$-linear $G$-action, so that we have a continuous homomorphism

$$
r: G \rightarrow \operatorname{Aut}_{\mathcal{K}}(V) \cong \mathrm{GL}_{d}(\mathcal{K}) .
$$

For example, take $\mathcal{O}$ to be $\mathbf{Z}_{p}$. Or, for another example, take it to be the power series $\operatorname{ring} \mathbf{F}_{p}[[t]]$, where we view the continuous $G$-representation $G \rightarrow \mathrm{GL}_{d}\left(\mathbf{F}_{p}[[t]]\right)$ as a formal variation of representations; here $t$ is the parameter variable (in the spirit of Figure 3 in Section 14) 39

Lemma 2. Let $G$ be a profinite group and $V$ a finite-dimensional $\mathcal{K}$-vector space endowed with a continuous $\mathcal{K}$-linear $G$-action, $r: G \rightarrow \operatorname{Aut}_{\mathcal{K}}(V)$.

(1) There is an $\mathcal{O}$-lattice $M \subset V$ that is $G$-stable.

(2) For each $g \in G$ the characteristic polynomial $\operatorname{det}(1-r(g) T)$ of the action of $g$ in the representation $r$ has coefficients in $\mathcal{O}$.

(3) If $M \subset V$ is a $G$-stable $\mathcal{O}$-lattice, form the associated "residual representation", $r_{M \otimes \kappa}$, i.e., the representation of $G$ on the $\kappa$-vector space $M \otimes_{\mathcal{O}} \kappa$ obtained from $r$ via the reduction $M \rightarrow M \otimes_{\mathcal{O}} \kappa$. Then for each $g \in G$ the characteristic polynomial

$$
\operatorname{det}\left(1-r_{M \otimes \kappa}(g) T\right)
$$

of the action of $g$ in the representation $r_{M \otimes \kappa}$ is the reduction of $\operatorname{det}(1-r(g) T)$ under the natural homomorphism $\mathcal{O}[T] \rightarrow \kappa[T]$.

(4) If $M, M^{\prime} \subset V$ are two $G$-stable $\mathcal{O}$-lattices, the Jordan-Hölder constitutent $\$ 0$ of the representations $r_{M \otimes \kappa}$ and $r_{M^{\prime} \otimes \kappa}$ are equal.

\footnotetext{
${ }^{38}$ One way of treating this ambiguity in "filling in" is to use Wiles' notion of a pseudo-character or pseudo-representation; see for example the opening sections of [6] for an exposition, and also see Section 1.1 of [31]. A very useful comprehensive discussion of the deformation theory of pseudo-representations (viewed as generalized determinants) and a primer on the background of the subject, making extensive use of the earlier work of Procesi and others, can be found in 16.

${ }^{39}$ We will also encounter later in this article variations of a slightly different order of complexity, coming from representations, say, to $\mathrm{GL}_{d}\left(\mathbf{Z}_{p}[[t]]\right)$ where wrench-type techniques are useful.

${ }^{40}$ That is, the irreducible representations occurring as subquotients of a Jordan-Hölder filtration, including their multiplicities.
} 
Proof. Starting with any lattice $M^{\prime} \subset V$, we will correct it to be $G$-stable. Note that the action $G \times M^{\prime} \rightarrow V$ is continuous, $M^{\prime}$ is finitely generated over $\mathcal{O}$, and $M^{\prime} \subset V$ is open. It follows that the subgroup $G_{o} \subset G$ stabilizing $M^{\prime}$ is an open subgroup of $G$. Since $G$ is profinite, we then have that $G_{o} \subset G$ is of finite index, and therefore $G$ stabilizes $M:=\sum g_{i} \cdot M^{\prime} \subset V$ where $\left\{g_{i}\right\}_{i}$ is a (finite) system of representatives of left $G_{O^{-}}$-cosets in $G$. This establishes (1).

The proofs of (2) and (3) are straightforward from this, while the final item (4) is an application of the Brauer-Nesbitt theorem (see [8], and also pp. 215-217 as well as Chapter XII of [18]).

In view of this lemma, given a (finite-dimensional) $G$-representation $\rho$ on a $\mathcal{K}$ vector space, we may speak of the various residual representations attached to $\rho$, these being representations of $G$ into $\kappa$-vector spaces obtained as the reduction of the various $G$-stable $\mathcal{O}$-lattices in the underlying $\mathcal{K}$-vector space of the representation $\rho$. We may also speak of the residual irreducible constituents of $\rho$ with their multiplicities, these being the irreducible representations that occur as JordanHölder constituents of one (or, equivalently, any) residual representation attached to $\rho$.

Here is the lemma that is the backbone of the "wrenching" strategy, expressed in general terms following Joël Bellaïche's article, A propos d'un lemme de Ribet (4]). We keep to the above terminology and hypotheses, fixing $\pi \in \mathcal{O}$ a uniformizer.

Lemma 3 (Ribet-Bellaïche). Let $G$ be a profinite group and $V$ a finite-dimensional $\mathcal{K}$-vector space endowed with a continuous irreducible $\mathcal{K}$-linear $G$-action, $r: G \rightarrow$ $\operatorname{Aut}_{\mathcal{K}}(V)$. Let $\tilde{M} \subset V$ be a $G$-stable $\mathcal{O}$-lattice so that we may view $r$ as a homomorphism $_{\tilde{M}}: G \rightarrow \operatorname{Aut}_{\mathcal{O}}(\tilde{M})$ and let $r_{\tilde{M} \otimes \kappa}: G \rightarrow \operatorname{Aut}_{\kappa}\left(\tilde{M} \otimes_{\mathcal{O}} \kappa\right)$ be the corresponding residual representation.

Now let $\bar{r}_{0}$ be a proper subquotient $\kappa[G]$-module in this residual representation $r_{\tilde{M} \otimes \kappa}$. Here "proper" means that the degree of $\bar{r}_{0}$ (i.e., the dimension of the underlying $\kappa$-vector space) is positive and strictly less than the degree of $r$ over $\mathcal{K}$ (equivalently, the degree of $r_{M \otimes \kappa}$ over $\kappa$ ).

Then there is a $G$-stable $\mathcal{O}$-lattice $M \subset V$ such that the associated residual representation $\bar{r}=r_{M \otimes \kappa}$ is isomorphic to a nonsplit extension of $G$-representations of $\kappa$-vector spaces, displayed here in terms of the labels of the corresponding representations:

$$
0 \rightarrow \bar{r}_{1} \longrightarrow \bar{r} \stackrel{\psi}{\longrightarrow} \bar{r}_{0} \rightarrow 0 .
$$

(Here "nonsplit" means that there is no $\kappa[G]$-equivariant homomorphism $\bar{r}_{0} \rightarrow \bar{r}$ that is a left-inverse to $\psi$.)

Remark. Ribet's original result was formulated when $\bar{r}_{1}, \bar{r}_{0}$ above are characters, i.e., representations of degree one. A proof of this theorem for $\bar{r}_{1}, \bar{r}_{0}$ a pair of distinct absolutely irreducible representations of higher degree is due to Urban, in [70. See also Theorem 1.1 of [71] for a different proof and see loc. cit. for remarks about the situation where $\bar{r}$ has more than two irreducible constituents.

Proof. By lattice (for short) we mean a $G$-stable $\mathcal{O}$-lattice in $V$. It is sometimes convenient to label the $\kappa[G]$-modules in the discussion below by the terms for the corresponding representations (e.g., $r_{\tilde{M} \otimes \kappa}$ and $\tilde{M} / \pi \tilde{M}$ are synonyms). 
Step 1: The initial wrench, moving $\bar{r}_{0}$ to a quotient of the residual representation. Since $\bar{r}_{0}$ occurs as a subquotient of the residual $G$-representation $\tilde{M} \otimes \kappa$, we may find a $G$-subrepresentation $\bar{N} \subset \tilde{M} \otimes \kappa$ such that $\bar{r}_{0}$ occurs as a quotient of $\bar{N}$. Let $M^{(0)} \subset \tilde{M}$ be the full inverse image in $\tilde{M}$ of $\bar{N} \subset \tilde{M} \otimes \kappa$ under the reduction homomorphism $\tilde{M} \rightarrow \tilde{M} \otimes \kappa$. Let $\bar{r}^{(0)}$ denote the residual representation $r_{M^{(0)} \otimes \kappa}$ associated to this new lattice $M^{(0)}$. We now have that $\bar{r}_{0}$ is a quotient of $\bar{r}^{(0)}$. Denote the kernel of the projection $M^{(0)} \otimes \kappa \rightarrow \bar{r}^{(0)}$ by $\bar{r}_{1}$. Consider the exact sequence of $\kappa$-representations

$$
0 \rightarrow \bar{r}_{1} \rightarrow \bar{r}^{(0)} \rightarrow \bar{r}_{0} \rightarrow 0 .
$$

Note that $\bar{r}_{1}$ is a representation of positive degree, given our hypothesis.

If this exact sequence, $(\mathbf{1})$, of $\kappa[G]$-modules is nonsplit, then we are done, so, to continue, suppose that we have a splitting of (1) (as a $G$-representation). Fix such a splitting, i.e., a direct sum decomposition

$$
\bar{r}^{(0)} \simeq \bar{r}_{1} \oplus \bar{r}_{0}
$$

Step 2: The inductive sequence of wrenches seeking a nonsplit extension. Let $M^{(1)} \subset M^{(0)}$ be the full inverse image in $M^{(0)}$ of the subrepresentation $\bar{r}_{0} \subset M^{(0)} \otimes \kappa$ under the reduction homomorphism $M^{(0)} \rightarrow M^{(0)} \otimes \kappa$. By construction, $M^{(1)}$ fits into two exact sequences of $G$-stable $\mathcal{O}$-modules:

$$
0 \rightarrow \pi M^{(0)} \rightarrow M^{(1)} \rightarrow \bar{r}_{0} \rightarrow 0
$$

and

$$
0 \rightarrow M^{(1)} \rightarrow M^{(0)} \rightarrow \bar{r}_{1} \rightarrow 0 .
$$

Consider, now, the residual representation, $\bar{r}^{(1)}=r_{M^{(1)} \otimes \kappa}$, associated to this new lattice $M^{(1)}$.

Lemma 4. This $\kappa[G]$-representation $\bar{r}^{(1)}$ fits into an exact sequence

$$
0 \rightarrow \bar{r}_{1} \rightarrow \bar{r}^{(1)} \rightarrow \bar{r}_{0} \rightarrow 0
$$

Proof. By definition of $M^{(1)}$ we have a surjection $M^{(1)} / \pi M^{(1)} \rightarrow \bar{r}_{0} \rightarrow 0$. Its kernel is canonically

$$
\pi M^{(0)} / \pi M^{(1)} \cong M^{(0)} / M^{(1)} \cong \bar{r}_{1}
$$

the latter isomorphism by (2) above.

Again, if this exact sequence $(\mathbf{3})$ of $\kappa[G]$-modules is nonsplit, we are done. Otherwise we continue the same procedure as above, writing

$$
\bar{r}^{(1)} \simeq \bar{r}_{1} \oplus \bar{r}_{0}
$$

(fixing a choice of splitting) and defining $M^{(2)} \subset M^{(1)}$ to be the full inverse image in $M^{(1)}$ of the subrepresentation $\bar{r}_{0} \subset M^{(1)} \otimes \kappa$ under the reduction homomorphism $M^{(1)} \rightarrow M^{(1)} \otimes \kappa$. Again, $M^{(2)}$ fits into two exact sequences of $G$-stable $\mathcal{O}$-modules:

$$
0 \rightarrow \pi M^{(1)} \rightarrow M^{(2)} \rightarrow \bar{r}_{0} \rightarrow 0
$$

and

$$
0 \rightarrow M^{(2)} \rightarrow M^{(1)} \rightarrow \bar{r}_{1} \rightarrow 0
$$

This gives us four things:

(1) An inclusion

$$
M^{(2)} \subset M^{(0)}
$$

with quotient $\mathcal{R}_{1}^{(2)}:=M^{(0)} / M^{(2)}$ being an extension of $\bar{r}_{1}$ by $\bar{r}_{1}$. 
(2) An inclusion $\pi^{2} M^{(0)} \subset M^{(2)}$. Its quotient, $\mathcal{R}_{0}^{(2)}:=M^{(2)} / \pi^{2} M^{(0)}$, is an extension of $\bar{r}_{0}=M^{(2)} / \pi M^{(1)}$ by

$$
\pi M^{(1)} / \pi^{2} M^{(0)} \cong M^{(1)} / \pi M^{(0)} \cong \bar{r}_{0}
$$

i.e., we have an exact sequence

$$
0 \rightarrow \bar{r}_{0} \rightarrow \mathcal{R}_{0}^{(2)} \rightarrow \bar{r}_{0} \rightarrow 0 .
$$

(3) The equality of lattices

$$
M^{(2)}+\pi M^{(0)}=M^{(1)} \subset M^{(0)},
$$

and (multiplying by $\pi$ )

$$
\pi M^{(2)}+\pi^{2} M^{(0)}=\pi M^{(1)} \subset M^{(2)} .
$$

(4) Combining these, we would get an exact sequence

$$
0 \rightarrow \mathcal{R}_{0}^{(2)} \rightarrow M^{(0)} / \pi^{2} M^{(0)} \rightarrow \mathcal{R}_{1}^{(2)} \rightarrow 0
$$

and, by (3), that $\mathcal{R}_{1}^{(2)} \otimes_{\mathcal{O}} \kappa \cong \bar{r}_{1}$ and $\mathcal{R}_{0}^{(2)} \otimes_{\mathcal{O}} \kappa \cong \bar{r}_{0}$.

Tensoring (4) over $\mathcal{O}$ with $\kappa$ yields an exact sequence

$$
\mathcal{R}_{0}^{(2)} \otimes_{\mathcal{O}} \kappa \rightarrow M^{(0)} / \pi M^{(0)} \rightarrow \mathcal{R}_{1}^{(2)} \otimes_{\mathcal{O}} \kappa \rightarrow 0,
$$

and since $M^{(0)} / \pi M^{(0)}=\bar{r}^{(0)}$, a consideration of dimensions over $\kappa$ shows that the homomorphism on the left above is injective, forming an exact sequence

$$
0 \rightarrow \bar{r}_{0} \rightarrow \bar{r}^{(0)} \rightarrow \bar{r}_{1} \rightarrow 0 \text {. }
$$

Step 3: An infinite sequence of sublattices. We discover in this manner that either we are done at some stage, or else we have an infinite sequence of $G$-stable sublattices

$$
\cdots \subset M^{(i+1)} \subset M^{(i)} \subset \cdots \subset M^{(0)}
$$

with the following properties.

(1) We have an exact sequence

$$
0 \rightarrow \pi^{i} M^{(0)} \rightarrow M^{(i)} \rightarrow \mathcal{R}_{0}^{(i)} \rightarrow 0
$$

where $\mathcal{R}_{0}^{(i)}$ has a Jordan-Hölder filtration of length $i$ with successive quotients isomorphic to $\bar{r}_{0}$.

(2) For $j \leq i$ we have that

$$
M^{(i)}+\pi^{j} M^{(0)}=M^{(j)} \subset M^{(0)} .
$$

(3) We have an exact sequence

$$
0 \rightarrow M^{(i)} \rightarrow M^{(0)} \rightarrow \mathcal{R}_{1}^{(i)} \rightarrow 0
$$

where $\mathcal{R}_{1}^{(i)}$ has a filtration of length $i$ with successive quotients isomorphic to $\bar{r}_{1}$.

(4) We have an exact sequence

$$
0 \rightarrow \mathcal{R}_{0}^{(i)} \rightarrow M^{(0)} / \pi^{i} M^{(0)} \rightarrow \mathcal{R}_{1}^{(i)} \rightarrow 0,
$$

and tensoring (5i) over $\mathcal{O}$ with $\mathcal{O} / \pi^{j} \mathcal{O}$ for any $j \leq i$ yields the exact sequence

$$
0 \rightarrow \mathcal{R}_{0}^{(j)} \rightarrow M^{(0)} / \pi^{j} M^{(0)} \rightarrow \mathcal{R}_{1}^{(j)} \rightarrow 0
$$


From (1) we get the inclusion

$$
\mathcal{R}_{0}^{(i)} \subset M^{(0)} / \pi^{i} M^{(0)},
$$

while from (2) we get that the natural projections $M^{(0)} / \pi^{i+1} M^{(0)} \rightarrow M^{(0)} / \pi^{i} M^{(0)}$ induce surjections

$$
\mathcal{R}_{0}^{(i+1)} \rightarrow \mathcal{R}_{0}^{(i)}
$$

From (3) we get surjections

$$
\mathcal{R}_{1}^{(i+1)} \rightarrow \mathcal{R}_{1}^{(i)} .
$$

Passing to the projective limits,

$$
\mathcal{R}_{0}:=\lim _{i} \mathcal{R}_{0}^{(i)} \subset \lim _{i} M^{(0)} / \pi^{i+1} M^{(0)}=M^{(0)}
$$

and

$$
\mathcal{R}_{1}:=\lim _{i} \mathcal{R}_{1}^{(i)},
$$

we get an exact sequence of $G$-stable $\mathcal{O}$-modules $0 \rightarrow \mathcal{R}_{0} \rightarrow M^{(0)} \rightarrow \mathcal{R}_{1} \rightarrow 0$. Under our assumption that $\bar{r}_{1}$ is proper, we get that both $\mathcal{R}_{0}$ and $\mathcal{R}_{1}$ are of infinite length, i.e., of positive rank, as $\mathcal{O}$-modules. Tensoring with $\mathcal{K}$ we then get that our original $\mathcal{K}$-representation $r$ has a proper $G$-stable subspace, contradicting the fact that it is assumed to be irreducible.

\section{A DIRECTED GRAPH}

Keeping the hypotheses of Section [15, let $r: G \rightarrow \operatorname{Aut}_{\mathcal{K}}(V)$ be an irreducible representation as in Lemma 3, and let $\bar{r}_{1}, \bar{r}_{2}, \ldots, \bar{r}_{\nu}$ be the irreducible residual constituents of $r$. Suppose for simplicity of notation that the $\bar{r}_{i}$ are all distinct; i.e., they each occur with multiplicity one in $r_{M \otimes \kappa}$ for any $G$-stable $\mathcal{O}$-lattice $M \subset$ $V$. Construct a graph $Y:=Y(r)$ as follows. The vertices of $Y$ are the residual constituents $\bar{r}_{1}, \bar{r}_{2}, \ldots, \bar{r}_{\nu}$. Draw a directed edge from $\bar{r}_{i}$ to $\bar{r}_{j}$ if there is a $G$-stable $\mathcal{O}$-lattice $M \subset V$ such that the associated residual representation $r_{M \otimes \kappa}$ possesses a subquotient which is a nontrivial extension of $\bar{r}_{i}$ by $\bar{r}_{j}$. Using the statement and proof of the above lemma, a further argument will show that the graph $Y$ is connected and each vertex of $Y$ has as least one directed edge leaving it, and another entering it 41 (It is easy enough to give examples where the graph is given by a single (directed) cycle lacing through all the residual irreducible constituents.)

The immediate effect of Ribet's strategy is to provide a large quantity of nontrivial extensions. The surprise is that (in many cases) one has, by application of this strategy, constructed extensions with very precise and useful further properties.

A major (but not the only) application of this lemma is when the residual representation has two distinct irreducible contitutents, $\bar{r}_{1}$ by $\bar{r}_{2}$, each occurring with multiplicity one. In this situation one obtains, given the hypotheses of Lemma 3 , two indecomposable residual representations: a nontrivial extension of $\bar{r}_{1}$ by $\bar{r}_{2}$ and

\footnotetext{
${ }^{41}$ If $Y$ is expressible as a disjoint union of two nonempty subgraphs, one first shows that for any lattice $M$ the associated residual representation $\bar{r}$ has a direct sum decomposition $\bar{r}=\bar{r}_{1} \oplus \bar{r}_{0}$, where each of the summands $\bar{r}_{1}, \bar{r}_{0}$ has irreducible constituents corresponding to the vertices of each of the two subgraphs. Moreover, under this hypothesis, for every lattice for which the associated residual representation $\bar{r}$ has a quotient representation isomorphic to $\bar{r}_{0}$, we have such a direct sum representation. The technique of the proof of Lemma 3 then works to show that this is impossible, thanks to the irreducibility of the $\mathcal{K}$-representation $r$. To show that each vertex of the graph has an edge directed to it (as well as emerging from it), apply Lemma 3 appropriately to the dual of $r$.
} 




Figure 4

a nontrivial extension of $\bar{r}_{2}$ by $\bar{r}_{1}$, giving us the elementary "complete" graph on two vertices shown in Figure 442

\section{A TREE}

Here is a somewhat more geometric view that one can adopt towards arguments similar to those in Sections 15 and 16.

Let us work, for example, with the case where the vector space $V$ is of dimension two over $\mathcal{K}$ and where $\kappa$ is a finite field, and where we have focused on a $\mathcal{K}$-linear continuous representation $r: G \rightarrow \operatorname{Aut}_{\mathcal{K}}(V)$. We say that two $\mathcal{O}$-lattices $M, M^{\prime}$ in $V$ are equivalen 43 if there is a nonzero element $\lambda$ of $\mathcal{K}$ such that $\lambda \cdot M=M^{\prime}$. Form the graph whose vertices are equivalence classes of $\mathcal{O}$-lattices, and two equivalence classes admit an edge between them if they have representatives $M, M^{\prime}$ such that $M \subset M^{\prime}$ with quotient $M^{\prime} / M$ an $\mathcal{O}$-module of length one (i.e., $M^{\prime} / M$ is a $\kappa$-vector space of dimension one).

The graph that we have just defined is a tree; call it $T$. This tree is acted upon naturally by $\operatorname{Aut}_{\mathcal{K}}(V) \cong \mathrm{GL}_{2}(\mathcal{K})$ with scalar matrices acting trivially, and so this action factors through the projective general linear group $\mathrm{PGL}_{2}(\mathcal{K})$. The representation $r$ induces then a continuous action of the profinite group $G$ on $T$.

Any $G$-stable lattice $M$ will represent a fixed vertex of this $G$-action. The converse is also true: any fixed vertex of this $G$-action is represented by $G$-stable $\mathcal{O}$-lattices 44

So the quest for $G$-stable $\mathcal{O}$-lattices is the same as the quest for the fixed point set $T^{G}$ in the tree $T$ under the action of $G$. For a beautiful exposition of this, see Section 6.6 of [58. Noting that the fixed point set in a tree under the action of a group must either be empty or a (sub)tre 45 and since, e.g., by (1) of Lemma 2 , $T^{G}$ is nonempty, we see that the collection $T^{G}$ of equivalence classes of $G$-stable $\mathcal{O}$-lattices in $V$ forms a subtree in $T$.

\footnotetext{
${ }^{42}$ Here is the argument that these nontrivial extensions, as elements of $\operatorname{Ext}_{k[G]}\left(\bar{r}_{1}, \bar{r}_{2}\right)$ and $\operatorname{Ext}_{k[G]}\left(\bar{r}_{2}, \bar{r}_{1}\right)$, are independent of the lattice for which they are residual representations: suppose that you have two lattices $M, M^{\prime}$ with indecomposable residual representations, $\bar{r}, \bar{r}^{\prime}$, both admitting, say, a $k[G]$-equivariant surjection to $\bar{r}_{2}$. Multiplying by an appropriate power of $\pi$, you can arrange it so that $M \subset M^{\prime}$ but $M$ is not contained in $\pi M^{\prime}$. Then either $M=M^{\prime}$ or else the image of $M$ in $\bar{r}^{\prime}$ is isomorphic to $\bar{r}_{2}$, thereby splitting $\bar{r}^{\prime}$ contrary to hypothesis.

${ }^{43}$ A synonym is "homothetic".

${ }^{44}$ This is because if $M$ represents a fixed vertex under the action of $G$ we get a natural continuous homomorphism from $G$ to the (discrete abelian group of) rational integers $\mathbf{Z}$ by the rule that assigns to $g \in G$ the unique $n \in \mathbf{Z}$ such that $g \cdot M=\pi^{n} \cdot M$. Such a mapping must be constant, and (since it is a homomorphism) it must be trivial.

${ }^{45}$ If $x$ and $y$ are vertices of the tree fixed under the action of the group $G$, then the unique geodesic between them is also fixed; hence $T^{G}$ is connected, if nonempty, and therefore a subtree (a connected subgraph of a tree is a tree).
} 
Each vertex $x$ of $T^{G}$ represents an equivalence class of $G$-stable lattices; the residual representations attached to the $\mathcal{O}$-lattices in this equivalence class are all canonically isomorphic (the isomorphisms are induced by multiplying lattices by an appropriate power of the uniformizer $\pi$ ). We refer to this representation over $\kappa$ as the residual representation attached to the vertex $x$.

The following proposition is proved by arguments similar to those in Lemma 3 .

Proposition 2. Let $r: G \rightarrow \operatorname{Aut}_{\mathcal{K}}(V)$ be a $\mathcal{K}$-linear continuous representation on a two-dimensional vector space $V$ over $\mathcal{K}$, and let $T$ be the associated tree of lattices.

(1) The subtree $T^{G}$ consists of a single vertex if and only if the residual representation $\bar{r}$ of $r$ is irreducible.

(2) From now on, suppose $T^{G}$ is not a single point, so the residual representation over $\kappa$ attached to any of the vertices of $T^{G}$ is reducible. A vertex is an extremal point in the tree $T^{G}$ if and only if the residual representation over $\kappa$ attached to it is (reducible and) indecomposable.

(3) Now let $G$ act on $V$ in a manner such that the semisimplification of its residual representation is a sum of two distinct (one-dimensional) characters $\alpha, \beta: G \rightarrow \kappa^{*}$.

(a) The subtree $T^{G}$ is linear and is equivalent to one of the following three subgraphs of the real line $\mathbf{R}$ (with vertices the integers):

(i) the full real line $(-\infty,+\infty)$,

(ii) the half-line $[0,+\infty)$,

(iii) a finite closed interval $[0, N]$ (for some $N \geq 1$ ).

The first case occurs if and only if the representation $r: G \rightarrow \mathrm{GL}_{2}(\mathcal{K})$ is a sum of two one-dimensional characters $A, B: G \rightarrow \mathcal{K}^{*}$.

The second case occurs if and only if the representation $r: G \rightarrow \mathrm{GL}_{2}(\mathcal{K})$ is a reducible indecomposable representation.

The third case occurs if and only if $r$ is irreducible. (So, in the first case, no G-stable lattice has an indecomposable residual representation; in the second case there is exactly one equivalence class of G-stable lattices possessing indecomposable residual representations; in the third case, there are exactly two.)

(b) Now suppose we are in the third case above, i.e., $r$ is irreducible. The two endpoints of the graph $T^{G}$ correspond to G-stable $\mathcal{O}$-lattices in $V$ whose residual representations are indecomposable and one of these residual representations will have a one-dimensional subrepresentation with the character $\alpha$; the other will have character $\beta$.

(c) The following are equivalent:

(i) The graph $T^{G}$ consists precisely of two (end-)vertices and the edge between them.

(ii) There is no $G$-stable $\mathcal{O}$-lattice in $V$ whose residual representation is semisimple.

(iii) There is no inclusion of $G$-stable $\mathcal{O}$-lattices $M \subset M^{\prime}$ in $V$ such that the quotient $M^{\prime} / M$ is a cyclic $\mathcal{O}$-module of length two.

For example, consider the representation $\rho_{\Delta, 691}$ of $G=G_{\mathbf{Q}}$ discussed in Section 13. By Proposition 1 in Section [12 its subtree $T^{G_{\mathbf{Q}}}$ is homeomorphic to the interval $[0,1]$ with the endpoints being its two vertices. 


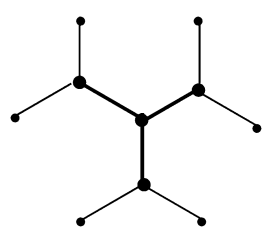

FiguRE 5

For more about this, see Section 24 below. For more examples, but still keeping to the modular form $\Delta$, one might move from the prime 691 to the prime 2 (or other "exceptional primes"; cf. 68, 69]) and the exercise of determining the corresponding subtrees fixed by $G_{\mathbf{Q}}$ under $\rho_{\Delta, 2}$ in the tree of 2-adic lattices.

For another kind of example, for the prime 2, let $G \subset \mathrm{GL}_{2}\left(\mathbf{Z}_{2}\right)$ be the kernel of the reduction homomorphism $\mathrm{GL}_{2}\left(\mathbf{Z}_{2}\right) \rightarrow \mathrm{GL}_{2}\left(\mathbf{F}_{2}\right)$, and the action of $G$ on $V:=\mathbf{Q}_{2} \times \mathbf{Q}_{2}$ be the standard action. Then $T^{G} \subset T$ consists of the thickened "Y" in the ambient infinite tree $T$, the star of this " $Y$ " in $T$ being depicted in Figure 5. The central vertex corresponds to the standard lattice $\mathbf{Z}_{2} \times \mathbf{Z}_{2} \subset \mathbf{Q}_{2} \times \mathbf{Q}_{2}=V$, the residual representation being trivial, and the three extremal vertices of the "Y" correspond to $G$-stable $\mathcal{O}$-lattices whose residual representations are reducible and indecomposable.

A consequence of Proposition 2 is the following result, which is very useful to the topic of this article.

Corollary 5. Let $r: G_{\mathbf{Q}} \rightarrow \operatorname{Aut}_{\mathcal{K}}(V)$ be an irreducible $\mathcal{K}$-linear continuous representation on a two-dimensional vector space $V$ over $\mathcal{K}$ such that:

(1) The residual representation(s) of $r$ are reducible, and their semisimplifications are a sum of two distinct (one-dimensional) characters $\alpha, \beta: G \rightarrow \kappa^{*}$;

(2) The restriction of $r$ to an inertia group attached to the prime $p$ is reducible.

Then there are exactly two equivalence classes of $G_{\mathbf{Q}}$-stable $\mathcal{O}$-lattices in $V$ with indecomposable residual representations. At least one of these two indecomposable residual representations, $\bar{r}$, has the added property that its restriction to any inertial group attached to $p$ is semisimple.

Proof. Let $I \subset G_{\mathbf{Q}}$ denote an inertia group attached to the prime $p$, and let $r_{I}: I \rightarrow$ $\operatorname{Aut}_{\mathcal{K}}(V)$ be the restriction of $r$ to $I$. Let $T$ be as above. By 3(a) of Proposition 2 above applied to the representation $r_{I}$ of $I$ we have that the subtree fixed by $I, T^{I}$, is either a full line or a half-line (depending on whether $r_{I}$ is semisimple or not). By 3(b) of Proposition 2 above applied to the representation $r$ of $G_{\mathbf{Q}}$ we have that the fixed subtree $T^{G_{\mathbf{Q}}} \subset T^{I}$ is a finite line. There are three different possibilities for this inclusion of trees, roughly indicated by the following figure, where the finite subtree $T^{G_{\mathbf{Q}}}$ is the darkened subgraph. In the top two cases, both endpoints of $T^{G_{\mathbf{Q}}}$ correspond to residual representations whose restrictions to inertia groups at the prime $p$ are semisimple, while in the last case, only one of the two residual representations attached to $r$ has that property.

For example, the representation $r_{\Delta, 691}$ has the properties (1) and (2) required of Corollary 5 , as do the representations associated to the modular forms $\Phi_{w, 691}$ 


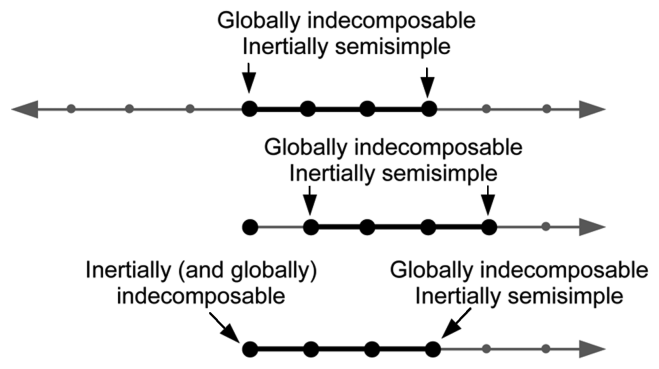

Figure 6. Here is what the trees of inertially and globally invariant lattices (represented by the the lighter tree and darker subtree, respectively) might look like when the global representation is as in 3.a(iii) of Proposition 2, and its restriction to an inertia group is as in 3.a(i) or 3.a(ii).

of Section 13 for $w \geq 2$. In fact, these Galois representations are ordinary 46 in the sense to be explained in Part IV, Section 20, see Definition 1.

Corollary 6. The two indecomposable residual representations attached to $r_{\Delta, 691}$ have the property that the restriction of one of them to an inertia group at $p$ is semisimple, and the other is not. The same is true for the two indecomposable residual representations attached to the representations $r_{w, 691}$ for any $w \geq 2$.

In particular, given what we have discussed so far, the pair of fixed subtrees $T^{G_{\mathbf{Q}}} \subset T^{I}$ for $r_{\Delta, 691}$ is isomorphic to $[0,1] \subset[0,+\infty)$ with the vertices being integers.

Corollary 6 follows from Corollary 5 together with the fact that although there is an element $z$ of order 691 in the ideal class group of $\mathbf{Q}\left(e^{2 \pi i / p}\right)$ such that if

$$
\operatorname{Gal}\left(\mathbf{Q}\left(e^{2 \pi i / p}\right) / \mathbf{Q}\right) \stackrel{\iota}{\longrightarrow} \mathbf{F}_{p}^{*}
$$

is the natural isomorphism, we have the formula

$$
\alpha(z)=\iota(\alpha)^{691-\nu} \cdot z=\iota(\alpha)^{1-\nu} \cdot z
$$

for $1-\nu=-11$. But there is no such element satisfying this condition for $1-\nu=$ +11 and therefore the other indecomposable residual representation does not have the property that its restriction to an inertia group at $p$ is semisimple.

\section{RAMificATiOn AT $p$ AND A "ClassicAl EXAMPlE"}

At this point we will look more closely at the issue hinted at in the $4 \Rightarrow \mathbf{3}$ lap in the discussion-of-proof at the end of Part I above. Why is the extension described in $\mathbf{3}$ everywhere unramified?

Our representation $\bar{\rho}$ is unramified at primes $\ell$ different from $p$ since $\bar{\rho}$ is contained in the Galois representation on $p$-torsion in the abelian variety $J_{1}(p) / J_{0}(p)$ which has good reduction outside the prime $p$ (see the discussion in Section 11) 47 To show

\footnotetext{
${ }^{46}$ This follows from the fact that their $U_{691}$-eigenvalue is nonzero mod 691 ; the eigenvalue is, in fact, congruent to $1 \bmod 691$.

${ }^{47}$ Good reduction outside $p$ would also follow from our discussion regarding $\bar{\rho}$ as related to Deligne's Theorem (see Section 9) since, for all $\ell \neq p$, Deligne obtains the representation from the Galois representation on the $\bmod p$ étale cohomology of an abelian variety over $\mathbf{F}_{\ell}$.
} 
that $\bar{\rho}$ is everywhere unramified we must show it to be unramified at the delicate prime $p$.

This follows by wrenching the situation in such a way that the global representation admits one type of splitting and the representation restricted to a decomposition group at $p$ admits a contrasting splitting.

I will illustrate this first in a situation that is significantly different from the above, but has the advantage of being one of the most studied examples among modular curves. Namely, consider the three isomorphically distinct, but isogenous, elliptic curves over $\mathbf{Q}$ of conductor 11. Two of these elliptic curves have standard names, $X_{1}(11)$ and $X_{0}(11)$; call the third (only in this article) $X_{-1}(11)$. These elliptic curves are linked by 5 -isogenies defined over $\mathbf{Q}$ :

$$
X_{-1}(11) \rightarrow X_{0}(11) \rightarrow X_{1}(11)
$$

and

$$
X_{1}(11) \rightarrow X_{0}(11) \rightarrow X_{-1}(11) .
$$

Here, then, $p=5, \kappa=\mathbf{Q}_{5}, \kappa=\mathbf{F}_{5}$, the group in question is $G=G_{\mathbf{Q},\{5,11, \infty\}}$, the Galois group over $\mathbf{Q}$ of the maximal algebraic extension that is unramified outside the places 5,11, and $\infty$, and the representation $r: G_{\mathbf{Q},\{5,11, \infty\}} \rightarrow \mathrm{GL}_{2}\left(\mathbf{Q}_{5}\right)$ comes from the action of Galois on the 5-power torsion subgroup of these elliptic curves. The two indecomposable residual representations are the representations of Galois on the 5-torsion of the two other elliptic curves over $\mathbf{Q}$ of conductor 11.

Setting $p=5$ in this discussion, fix a decomposition group relative to the prime $p=5$,

$$
D_{p}=D_{5} \subset G_{\mathbf{Q},\{5,11, \infty\}}
$$

and consider the $G:=G_{\mathbf{Q},\{5,11, \infty\}}$-module of $p$-torsion points $E[p]$ for each of our three elliptic curves. These are vector spaces of dimension two over $\mathbf{F}_{p}$ with $\mathbf{F}_{p}$-linear $G$-actions.

- When $E=X_{0}(11)$, the intermediate elliptic curve in the two chains above, the $G$-action is (reducible, and) semisimple, and we have

$$
E[p] \approx \mathbf{Z} / p \mathbf{Z} \oplus \mu_{p}
$$

where the $G$-action on $\mathbf{Z} / p \mathbf{Z}$ is trivial, and on $\mu_{p}$ is the natural Galois action on $p$ th roots of unity. Division by "the $\mathbf{Z} / p \mathbf{Z}$ " yields $X_{-1}(11)$ while division by "the $\mu_{p}$ " yields $X_{1}(11)$.

- Now, given what we have said above, when $E:=X_{1}(11)$ (and, as in this entire discussion, $p=5$ ) we have the exact sequence of $G$-representations,

$$
\text { (*) } \quad 0 \rightarrow \mathbf{Z} / p \mathbf{Z} \rightarrow E[p] \rightarrow \mu_{p} \rightarrow 0 .
$$

But (for finite flat group scheme reasons) when you restrict the action to the decomposition group at $5, D_{p} \subset G$, you find the $D_{p}$-module $E[p]$ fitting into an exact sequence where the $\mathbf{Z} / p \mathbf{Z}$ and $\mu_{p}$ occur in the opposite order 48

\footnotetext{
${ }^{48}$ Here, briefly, is the reason for this. Viewing the $D_{p}$-representation space $E[p]$ as the Galois module at the generic point of $S:=\operatorname{Spec}\left(\mathbf{Z}_{p}\right)$ of a finite flat group scheme $\mathcal{E}$ over $S$ we may fit $\mathcal{E}$ into a canonical exact sequence of finite flat group schemes,

$$
(* *) \quad 0 \rightarrow \mathcal{E}^{o} \rightarrow \mathcal{E} \rightarrow \mathcal{E}^{e t} \rightarrow 0,
$$

where $\mathcal{E}^{o}$ is the open connected component finite flat subgroup scheme of $\mathcal{E}$, and $\mathcal{E}^{e t}$ is the maximal étale quotient of $\mathcal{E}$. Now pass to the Galois modules associated to the generic fiber of these finite flat group schemes. Given the standard results regarding finite flat group schemes over $S$ (since
} 
Since the $D_{p}$-representations $\mathbf{Z} / p \mathbf{Z}$ and $\mu_{p}$ are distinct, this provides the wrench showing that $D_{p}$ acts semisimply on $E[p]$ (this $D_{p}$-representation being then just the direct sum $\mathbf{Z} / p \mathbf{Z} \oplus \mu_{p}$ ). In particular, if $L / \mathbf{Q}$ is the splitting field (over $\mathbf{Q}$ ) of the $G_{\mathbf{Q}}$-representation $E[p]$ when $E=X_{1}(11)$, then $L / \mathbf{Q}\left(e^{2 \pi i / 5}\right)$ is unramified at $p$. Since $E[p]$ is also indecomposable as a $G_{\mathbf{Q}}$-representation, $L$ will be a cyclic degree $p$ extension unramified at $p$ and only ramified at primes above 11 .

- When $E=X_{-1}(11)$ the $G$-representation $E[p]$ fits into an exact sequence

$$
0 \rightarrow \mu_{p} \rightarrow E[p] \rightarrow \mathbf{Z} / p \mathbf{Z} \rightarrow 0,
$$

so the local (finite flat group scheme) argument above gives us no further information in this case.

A direct computation, however, of the 5-division field of $E=X_{-1}(11)$ (performed by William Stein using SAGE) tells us that this field is wildly ramified over $\mathbf{Q}\left(\mu_{5}\right)$, and, in particular, the inertia group $I \subset G$ at 5 acts (reducibly, but) in an indecomposable (i.e., nonsemisimple) manner on $E[p]$.

It follows that (in the terminology of Section 17) the tree $T^{I}$ fixed by the inertia group $I$ at the prime $p=5$ for the elliptic curve $E=X_{-1}(11)$ is the half-infinite line $[0, \infty)$, and the subtree $T^{G} \subset T^{I}$ that is fixed by $G=G_{\mathbf{Q},\{5,11, \infty\}}$ consists of the interval $[0,2] \subset[0, \infty)$.

Ribet employs this argument not for elliptic curves of this sort, or for elliptic curves exclusively, but rather for abelian varieties over $\mathbf{Q}$ of the form $J_{1}(p) / J_{0}(p)$ (or abelian varieties isogenous to quotients of $J_{1}(p) / J_{0}(p)$ ), these having good reduction outside of $p$. Therefore, ramification at $p$ is the only issue in question here. But these abelian schemes actually achieve good reduction also at $p$ over the field $\mathbf{Q}\left(\mu_{p}\right)^{+}$(cf. [20]), which is enough to press an analogue of the above argument forward 49

\section{LiminAL REPRESENTATIONS}

In Sections 14 and 15 we focused on the phenomenon of irreducible representations degenerating to reducible ones. One can also consider the prospect of "going the other way". That is, given a reducible representation $\rho_{0}$ of $G$ in, say, a finitedimensional $\mathbf{Q}_{p}$-vector space, when can we find a (locally analytic, say) family of representations $\rho_{t}$ parametrized by a variable $t$ ranging through a disc about the origin such that:

- the generic member of the family is an irreducible representation of $G$; and

- the specialization of this family to $t=0$ is our initial representation $\rho_{0}$ ?

If the above happens (given, say, by a continuous homomorphism $\rho: G \rightarrow$ $\mathrm{GL}_{2}\left(\mathbf{Z}_{p}[[X]]\right)$, where $\rho_{t}$ is the composition of $\rho$ with the homomorphism induced

$p=5>2$ ) we get that, given $(*)$ above, $\mathcal{E}^{o}$ is necessarily the "multiplicative" group scheme $\mu_{p}$ over $S$ and $\mathcal{E}^{\text {et }}$ is the constant group scheme $\mathbf{Z} / p \mathbf{Z}$ over $S$. Comparison with (**) gives us our splitting of $D_{p}$-modules:

$$
0 \rightarrow \mu_{p} \rightarrow E[p] \rightarrow \mathbf{Z} / p \mathbf{Z} \rightarrow 0
$$

\footnotetext{
${ }^{49}$ More specifically, working with such an abelian scheme locally over the completion of $\mathbf{Z}\left(\mu_{p}\right)^{+}$ at the prime above $p$, one uses a crucial result of M. Raynaud (48]) that gives information about the structure of finite flat subgroup schemes of exponent $p$ over discrete valuation rings that are finite degree extensions of $\mathbf{Z}_{p}$ of ramification index $<p-1$ together with a wrenching argument very similar to the one in the previous paragraph.
} 
by specializing $X \mapsto t$ ), we will call the representation $\rho_{0}$ limit-irreducible, or for short, liminal.

When we say that a given representation $\rho: G \rightarrow \mathrm{GL}_{2}\left(\mathbf{Z}_{p}\right)$ is the limit of representations satisfying a particular property $P$ we mean, more explicitly, that there is a sequence $\rho_{i}: G \rightarrow \mathrm{GL}_{2}\left(\mathbf{Z}_{p}\right)$ of continuous homomorphisms, each satisfying property $P$ (for $i=1,2,3, \ldots$ ) converging in the $p$-adic topology to $\rho$. We will usually want our family $\rho_{t}$ to have some specifically described good properties, and we hope that in certain good situations we can predict that the liminal representation has the same good properties 50

A (technically only slightly) different version of this concept of liminality is when there is a complete discrete-valued field $\mathcal{K}$, as in the previous discussion, with residue field $\kappa$, and for which there is a continuous irreducible representation $\rho$ of $G$ into a finite-dimensional $\mathcal{K}$-vector space having $\rho_{0}$ as one of its residual $\kappa$-vector space representations.

\section{ORdinARY AND NEARLY ORDINARY GALOIS REPRESENTATIONS}

An extremely useful thing to do, in order to learn important facts about the restriction to a decomposition group at $p$ of the $p$-adic Galois representation attached to an eigenform $\Phi$ (such as $\Delta$ or the eigenforms $\Phi_{w}$ we have been discussing), is to determine whether the eigenvalue of the Atkin-Lehner operator, $U_{p}$, is congruent to zero or not modulo $p$. If it is not congruent to zero mod $p$ one says that the eigenform is p-ordinary, and one learns that the restriction to a decomposition group at $p$ of the $p$-adic Galois representation attached to $\Phi$ is reducible (cf. [43], [75] 51

We will now frame this discussion in a more general context regarding a sequence of conditions on degree two Galois representations over number fields.

Let $K$ be a number field, $\bar{K} / K$ an algebraic closure, and put $G_{K}:=\operatorname{Gal}(\bar{K} / K)$. Let $S$ be a finite set of places of $K$, and let $G_{K, S}$ be the quotient of $G_{K}$ obtained by dividing by the closed normal subgroup generated by all inertia groups for places outside $S$. Fix $p$ a prime, let $S(K, p)$ denote the set of all places of $K$ dividing $p$, and suppose that $S$ contains $S(K, p)$.

Let $M$ be a free $\mathbf{Z}_{p}$-module of rank two endowed with a continuous $\mathbf{Z}_{p}$-linear action of $G_{K, S}$. Let

$$
\rho: G_{K, S} \longrightarrow \operatorname{Aut}(M) \simeq \mathrm{GL}_{2}\left(\mathbf{Z}_{p}\right)
$$

denote the corresponding degree two representation.

\section{Definition 1.}

(1) We say that $\rho$ is nearly ordinary if for all $v \in S(K, p)$ the restriction $\rho_{v}$ of $\rho$ to a decomposition group $G_{K_{v}}$ at $v$ preserves a free rank one submodule $M_{v} \subset M$. We can, and do, take $M_{v}$ to be saturated in $M$; we choose a decomposition subgroup for $v$ and such an $M_{v}$ for each $v \in S(K, p)$ and,

\footnotetext{
${ }^{50}$ In a more general, yet still particular, context, a big role in recent developments is played by a theorem of Kisin that allows us to deduce that the liminal representation $\rho$ possesses a limiting $p$-adic period in the sense of Fontaine's theory, provided the approximating representations $\rho_{i}$, when restricted to the decomposition group at $p$, satisfy appropriate requirements (see [37] and Section 3 of [14]).

${ }^{51}$ In the case of $\Delta$ and the $\Phi_{w}$ 's, for example, when $p=691$ the eigenvalue of the Atkin-Lehner operator, $U_{691}$, is congruent to $1 \bmod 691$. Therefore, these eigenforms are $p$-ordinary, for $p=691$.
} 
in cases where there are more than one such possible $M_{v}$, we view these choices as part of the nearly ordinary structure of $\rho$; cf. [11. We then have for each $v \in S(K, p)$ an exact sequence of $G_{K_{v}}$-modules:

$$
0 \rightarrow M_{v} \rightarrow M \rightarrow M / M_{v} \rightarrow 0 .
$$

The restriction of $\rho_{v}$ to $M_{v}$ and the induced action on $M / M_{v}$ give us two degree one characters, i.e., homomorphisms $G_{K_{v}} \rightarrow \mathbf{Z}_{p}^{*}$, for each $v \in S(K, p)$. For evident reasons let us call the character giving the action on $M_{v}$ the local subcharacter of the nearly ordinary representation $\rho$ at $v$, and call the character giving the action on $M / M_{v}$ the local quotient-character.

(2) We say that a nearly ordinary (degree two) Galois representation is ordinary if the local quotient-characters of $\rho$ for all $v \in S(K, p)$ are unramified 52

(3) We say that an ordinary (degree two) Galois representation is anomalous if the local quotient characters of $\rho$ for all $v \in S(K, p)$ are trivial.

For reasons that will become apparent below, we will define Iwasawa representations to allow for more general scalars than the $\mathbf{Q}_{p}$ that was operative in the above definition. Consider a continuous Galois $\left(G_{K}\right)$ representation on a two-dimensional vector space $V$ over $\mathbf{C}_{p}: \rho: G_{K} \rightarrow \operatorname{Aut}_{\mathbf{C}_{p}}(V) \approx \mathrm{GL}_{2}\left(\mathbf{C}_{p}\right)$.

Definition 2. We will call such a representation an Iwasawa representation if

(1) the global representation $\rho$ is indecomposable;

(2) the semisimplication of $\rho$ is the sum of two characters of $G_{K}$, a nontrivial character $\chi: G_{K} \rightarrow \mathbf{C}_{p}^{*}$ and the trivial character $\mathbf{1}$;

(3) the character $\chi=\operatorname{det}(\rho)$ has the property that its minimal splitting field over $K$ is a $\mathbf{Z}_{p}$-extension of $K$;

(4) the character $\chi$ occurs as a $s u b$-representation of $\rho$ and the trivial character 1 occurs as a quotient-representation;

(5) for all $v \in S(K, p)$ the local representation $\rho_{v}$ when restricted to the inertia group $\mathcal{I}_{K_{v}} \subset G_{K_{v}}$ is semisimple 53

We are often, but not always, specifically interested in Iwasawa representations with determinant characters $\chi$ that cut out the p-cyclotomic $\mathbf{Z}_{p}$-extension of $K$.

Note that if an Iwasawa representation takes its values in $\mathrm{GL}_{2}\left(\mathbf{Z}_{p}\right)$ we may view it as a nearly ordinary representation, and indeed, for each $v \in S(K, p)$, we have our choice as to which of the two local degree one characters we choose to be the subcharacter, which the quotient-character. Choosing, consistently, the local quotient-character to be the trivial character as we can do by (5), the Iwasawa representation is then anomalous.

\footnotetext{
${ }^{52}$ There is a somewhat arbitrary choice to be made here: some texts define ordinary by the requirement that the local sub-characters of $\rho$ (for $v \in S(K, p)$ ) are all unramified. The two choices are elementarily related in that if a $\mathbf{Z}_{p}\left[G_{K}\right]$-module $M$ is ordinary according to one of these choices, its $\mathbf{Z}_{p}$-dual, $\operatorname{Hom}\left(M, \mathbf{Z}_{p}\right)$, will be ordinary according to the other. In effect, if you are dealing with cohomology, the choice we have just made is slightly more natural than the other, and if you are dealing with homology, the situation is reversed.

${ }^{53}$ If the local representation $\rho_{v}$ when restricted to the inertia group is semisimple it also follows, in this situation, that the local representation $\rho_{v}$ (on the entire decomposition group) is semisimple since (a) the inertia group at a prime $v$ is a normal subgroup of the corresponding decomposition group at $v$, and (b) under our hypotheses, the inertia group representation is a sum of two distinct characters.
} 


\section{PARAmeter spaces of ordinary Degree tWo Galois Representations}

Let us return to the context of the beginning of Section [15. Suppose you are given a family $\rho_{t}$ of degree two ordinary $p$-adic $G_{\mathbf{Q}}$-representations parametrized by an open disc in $\mathbf{Q}_{p}$, with parameter variable denoted $t$.

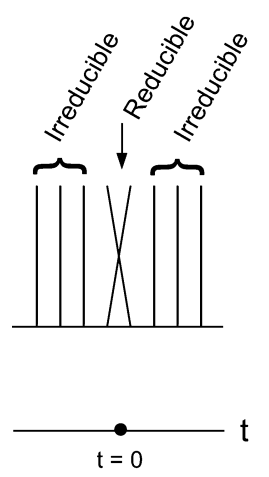

FiguRE 3. (repeated)

Suppose that these $\rho_{t}$ 's are generically irreducible in the sense described previously, and for the value $t=t_{o}$ the representation $\rho_{t_{o}}$ is not irreducible and therefore even though its semisimplification is well defined, as previously discussed, it itself is not well defined; suppose that it has as its Jordan-Hölder constituents the $G_{\mathbf{Q}^{-}}$ characters 1 and $\chi$, where $\chi$ is ramified at $p$. We may "fill in" the family in more than one way; we choose $\rho_{t_{o}}$ to be indecomposable and such that we have an exact sequence of $G_{\mathbf{Q}}$-representations with the following "ordering of characters":

$$
0 \rightarrow \mathbf{1} \rightarrow \rho_{t_{o}} \rightarrow \chi \rightarrow 0
$$

Now, since our family is ordinary, and $\chi$ is ramified at $p$, the $D_{p}$-representation obtained from $\rho_{t_{o}}$ also fits into an exact sequence of $D_{p}$-representations, but with the opposite ordering:

$$
0 \rightarrow \chi \rightarrow \rho_{t_{o}} \rightarrow \mathbf{1} \rightarrow 0
$$

The same wrench phenomenon applies as in the previous bullet to give us that if $K$ is the splitting field of $\chi$ over $\mathbf{Q}$, and $L$ is the splitting field of $\rho_{t_{o}}$ over $\mathbf{Q}$, then $K \subset L$ and $L / K$ is unramified at $p$ (interpreted appropriately, since these in general will be extension fields of infinite degree over $\mathbf{Q}$ ).

\section{LiminALity OF IWASAWA REPRESENTATIONS}

The proof of the main conjecture (42] over $\mathbf{Q}$; and more generally, 76 over totally real fields) tells us something about the liminality of Iwasawa representations. Specifically, in the special case over $\mathbf{Q}$, for $\chi$ a $p$-adic character of $G_{\mathbf{Q}}$ let $\chi^{*}=\epsilon \chi^{-1}$, where $\epsilon: G_{\mathbf{Q}} \rightarrow \mathbf{Z}_{p}^{*}$ is the $p$-cyclotomic character.

(1) The $p$-adic Leopoldt-Kubota $L$-function, $L_{p}(\xi)$, vanishes at the $p$-adic character $\xi=\chi^{*}$ if and only if there is an Iwasawa representation of determinant $\chi$. 
(2) There is one and only one Iwasawa representation of determinant $\chi$ if and only if the zero of $L_{p}$ is simple at $\chi^{*}$ and in this case the Iwasawa representation occurs as an indecomposable representation $\rho_{s}$ attached to a point $s$ on a one-parameter $p$-adic analytic family (technically: a Hida family) of generically irreducible degree two Galois representations.

A consequence is that if the zero of $L_{p}$ is simple at $\chi^{*}$, then the corresponding Iwasawa representation is indeed liminal in a very strong way: it is a limit of geometric, ordinary, irreducible representations 54

Sidenote. There is something baffling happening in the (unlikely) event that the Leopoldt-Kubota $L$-function has a multiple zero at $\chi^{*}$. For then we have a positivedimensional projective space parametrizing all Iwasawa representations of determinant $\chi$, and yet only finitely many of these will lie in Hida components. Which ones? Or are all zeroes of the Leopoldt-Kubota $L$-function simple 55

PART IV:

\section{Liminality in RECENT AND CURRENT Work}

\section{The General FRAMEWORK}

To succinctly remind ourselves of Ribet's idea, but framing it in the more general context of reductive groups, we may illustrate the procedure by these six steps (allowing for variants, the most evident variant being a congruence version of what we describe below, such as in the format originally used by Ribet himself) 56

(1) The initial equipment:

Let $p$ be a prime number and $K$ a number field.

Let $H^{(1)}, H^{(2)}, \ldots, H^{(\nu)}$ be a collection of reductive groups over $\overline{\mathbf{Q}}_{p}$. In practice, these groups $H^{(i)}$ will be either general linear, symplectic, or unitary groups, and we will be considering them as subgroups of general linear groups via their standard representations.

More to the point, we often also take the $H^{(i)}$ as the Langlands dual groups to reductive groups, $\mathcal{H}^{i}$, which are either general linear or are of symplectic or unitary type over $\mathbf{Q}$ or over totally real or CM number fields.

Let

$$
\rho^{(i)}: G_{K} \rightarrow H^{(i)}\left(\overline{\mathbf{Q}}_{p}\right)
$$

$(i=1,2, \ldots, \nu)$ be irreducible Galois representations 57

The above Galois representations will, in recent practice again, either be of degree one, i.e., characters, or, more generally, will be obtained from

\footnotetext{
${ }^{54}$ If $K$ is a totally real field (and, for simplicity of discussion, assume that Leopoldt's conjecture holds for $K$ ), then by the proof of the main conjecture for $K$ (cf. 76]) one sees that the same statement holds for Iwasawa representations over $K$.

${ }^{55}$ In the improperly irregular case we know that the maximal everywhere unramified $p$-abelian extension of $\bigcup_{n=1}^{\infty} \mathbf{Q}\left(e^{2 \pi i / p^{n}}\right)$ can be generated by the extraction of $p^{\nu}$ th powers of the appropriate subgroup of cyclotomic units in $\mathbf{Q}\left(e^{2 \pi i / p^{m}}\right)$ (for appropriate exponents $\nu$, as $m$ goes to $\infty$ ). It follows, in the improperly irregular case, that if the corresponding Iwasawa module is semisimple, then all the zeroes of the Leopoldt-Kubota $L$-function will be simple.

${ }^{56}$ The framework is similar when you are dealing with unitary groups and you have a quadratic extension $K / k$ in the works.

${ }^{57}$ By "irreducible" all I mean is that the composition of these representations with the standard representation yields an irreducible representation of $G_{K}$ into the corresponding general linear group.
} 
automorphic forms for $\mathcal{H}^{i}$. We will call these $\rho^{(i)}$ the constituent representations.

(2) The encompassing equipment:

We wish to find interesting $G_{K}$ representations that are extensions of appropriate pairs of the constituent representations $\rho^{(i)}(i=1,2, \ldots, \nu)$.

To this aim, one seeks

- some well-chosen reductive group $\mathcal{G}$ over a number field whose Langlands dual group, $G$, contains a parabolic subgroup $P \subset G$ with $P=H \cdot U$ a Levi decomposition, where $H=H^{(1)} \times H^{(2)} \times \cdots \times H^{(\nu)}$, and

- an automorphic form $\pi_{o}$ for $\mathcal{G}$ that has an associated Galois representation $\rho_{o}: G_{K} \rightarrow P\left(\overline{\mathbf{Q}}_{p}\right) \subset G\left(\overline{\mathbf{Q}}_{p}\right)$ and such that composition with the natural projection

$$
G_{K} \longrightarrow P\left(\overline{\mathbf{Q}}_{p}\right) \rightarrow H\left(\overline{\mathbf{Q}}_{p}\right)=\prod_{i=1}^{\nu} H^{(i)}\left(\overline{\mathbf{Q}}_{p}\right)
$$

is $\prod_{i=1}^{\nu} \rho^{(i)}$. One then picks out appropriate two-stage subquotient representations. We call the reductive group $\mathcal{G}$ "the" encompassing reductive group.

In the initial use, and in early applications, the automorphic form $\pi_{o}$ was taken to be an appropriate Eisenstein series, but certain other nontempered representations have also been brought into play, and the all-important passage from $\mathbf{6}$ to $\mathbf{5}$ in Ribet's original method, which bridges - via the convenience of congruences - the divide between Eisenstein series and cuspforms, is sometimes replaced by bridging the divide between nontempered and tempered automorphic representations, following a suggestion made many years ago by Harder.

(3) The automorphic form is "fit" into a p-adic family: "Fit" $\pi_{o}$ into a $p$ adically interpolable family of automorphic representations of the encompassing reductive group, and prove that there is indeed an associated (rigid analytic) family of Galois representations

$$
\rho_{t}: G_{K} \rightarrow G\left(\overline{\mathbf{Q}}_{p}\right)
$$

parametrized by an irreducible ("pointed") rigid analytic space $\left(T, t_{o}\right)$ such that

- for $t=t_{o}$, the semisimplification of $\rho_{t_{o}}$ is $\rho_{o}^{\text {ss }}$,

- for $t \neq t_{o}$, the $\rho_{t}$ are irreducible representations,

- for a Zariski-dense set of values of $t$, the $\rho_{t}$ 's have good properties.

(4) The wrench is used to get a desired liminal representation: Now use the lemma of Ribet-Bellaïche and the "wrench phenomenon" described earlier to modify, if necessary, the limiting $\rho_{t_{o}}$ so as to get an interesting liminal Galois representation; call it $\rho_{o}$. The number field cut out by the nonsemisimple representation $\rho_{o}$ constructs, for us, Galois extensions of the number field cut out by the original constituent representations, described in the Ribet-Bellaïche lemma.

(5) Establishing good properties of the action of the decomposition group: Finally, use whatever good properties the $\rho_{t}$ have when restricted to the 
decomposition groups at places above $p$ to guarantee good local behavior (at places above $p$ ) for $\rho_{o}$ and hence for the nontrivial extensions of the constituent representations that you have constructed, thereby showing that certain of these extensions provide sought-for elements in appropriate Selmer groups.

Here are a few examples to give a brief rough idea of the type of work that has been done 58 and that is being done, along these lines 59 Below, the symbol $\chi$ will just mean some Galois character to $\overline{\mathbf{Q}}_{p}^{*}=\mathrm{GL}_{1}\left(\overline{\mathbf{Q}}_{p}\right)$ but, in practice, one may also want to deal with characters to multiplicative groups of extension fields of $\overline{\mathbf{Q}}_{p}$.

(1) The above seems not to be too bad a strategy if you want to prove "main conjectures" as in 42] (the main conjecture of Iwasawa theory over $\mathbf{Q}$, and more generally as in [76], the main conjecture of Iwasawa theory over totally real fields). The large difference between the approaches in [42] and [76] is that (although both follow the Ribet wrench philosophy) [42] makes extensive and particular use of the algebraic geometric structure of the Jacobian of modular curves (which is not available in a more general setting) while 76 replaces this with the more automorphic format as described above. Here $\nu=2$, the reductive groups $H^{(1)}, H^{(2)}$ are both isomorphic to $\mathrm{GL}_{1}$, and the constituent representations are two characters, one of them the trivial character. The encompassing reductive group is $\mathrm{GL}_{2}$ and the parabolic subgroup $P \subset G$ is the Borel subgroup of upper triangular matrices. We can summarize this by the picture of the $2 \times 2$-matrix that we will think of as a $(1+1) \times(1+1)$ matrix

$$
\left(\begin{array}{ll}
1 & * \\
0 & \chi
\end{array}\right)
$$

the extension constructed (signified by the " $*$ in the upper right-hand corner") providing us with elements in the one-dimensional Galois cohomology of the degree one representation $\chi$ and, thanks to Step 5 above, these elements enjoy good local properties. The congruence version of this is, of course, the strategy initiated by Ribet. An appropriate Hida family gives the $p$-adically varying family of Galois representations " $\rho_{t}$ ".

(2) To construct elements in the Selmer group of an adjoint representation of a Galois representation $\rho: G_{K} \rightarrow \mathrm{GL}_{d}\left(\overline{\mathbf{Q}}_{p}\right)$ one might try the above strategy with $\rho$ and $\rho^{*} \otimes \chi$ as constituent characters, with $G=\mathrm{GSp}_{2 d}$, where the picture corresponding to the one drawn in (2), just above, is of the $2 d \times 2 d$-matrix

$$
\left(\begin{array}{cc}
\rho & * \\
0 & \rho^{*} \otimes \chi
\end{array}\right)
$$

and the parabolic subgroup $P \subset G$ is the evident one. This is the format described in 1997 by Haruzo Hida, Jacques Tilouine, and Eric Urban 32 in their strategy for a possible proof of the main conjecture for the adjoint representation $\operatorname{ad}(\phi)$, where $\phi$ is the $\mathrm{GL}_{2}$ automorphic representation attached to an elliptic curve over $\mathbf{Q}$. Here $d=2, G=\mathrm{GSp}_{4}$, and one then

\footnotetext{
${ }^{58}$ It is noteworthy, and natural, that many, perhaps all, of the classic results proving modularity of two-dimensional representations of $\operatorname{Gal}(\overline{\mathbf{Q}} / \mathbf{Q})$ (that satisfy appropriate hypotheses) make use of procedures that touch on Ribet's wrench; to cite one important example, see 63.

${ }^{59}$ I am thankful to Michael Harris for help in preparing this brief summary.
} 
may hope to use $p$-adic rigid analytic families of $\mathrm{GSp}_{4}$-automorphic forms to effect steps (2) and (3) of the strategy outlined above, and thereby to produce elements in the Selmer group of twists of the adjoint representation to $\rho$. This was formulated as a candidate strategy in [32, and many of the technical hurdles to carry it out were dealt with in that article. As Michael Harris has explained to me, what was principally left yet to be done (given [32]) to obtain this main conjecture was to guarantee nondivisibility by $p$ of (nonconstant) Fourier coefficients of certain Eisenstein series.

One has, in this story of the main conjecture for the adjoint representation $\operatorname{ad}(\phi)$, three basic objects: the $p$-adic $L$-function interpolating the critical values of the symmetric square of the modular forms in these families, the characteristic ideal of the associated Selmer group, and a characteristic Eisenstein ideal containing information on the congruences between cuspidal Siegel modular forms of genus 2 and the Klingen-type Eisenstein series. Regarding this, see [72], where, in an appropriate context, the divisibility of the Eisenstein ideal alluded to above by the $L$-function is shown, and see [71] for the divisibility of the characteristic ideal of the Selmer group by the Eisenstein ideal.

(3) Finding elements of the Selmer group when the sign of the functional equation would predict that they exist seems to be amenable to the above outlined approach.

(a) A "congruence version" of (1), i.e., a strategy close to Ribet's original strategy, was carried out by Joël Bellaïche for quadratic imaginary fields in his thesis [3] (cf. the recently published [2]), where for a set of primes $p$ of positive density, a Ribet-type theorem was proven relating nontriviality of the $p$-primary group of the Selmer group of algebraic Hecke characters over an imaginary quadratic field if the sign of the corresponding $L$-function is -1 . As alluded to above, Harder's suggestion (of replacing the Eisenstein series that played the role, in $\mathbf{6}$, of Ribet's proof by a CAP automorphic form 60 is employed by Bellaïche in his approach, coupled with an idea due to Clozel, Bellaïche's thesis advisor 61 Here $\nu=3$, the encompassing reductive group is a unitary group in three variables, and if $\eta$ is the algebraic Hecke character one is studying, the residual representation attached to the " $\rho_{o}^{\text {ss" }}$ of the above method is a sum of three characters with the residual representation attached to $\eta$ occurring as one of the constituents.

(b) Starting with a self-dual $G_{\mathbf{Q}}$-representation $\rho$ into $\mathrm{GL}_{2}\left(\overline{\mathbf{Q}}_{p}\right)$, and a character $\chi$, and again letting $\nu=3$, consider the triple of constituent $G_{\mathbf{Q}}$-representations $\chi, \rho, \chi^{*}$ (with values in $\mathrm{GL}_{1}\left(\overline{\mathbf{Q}}_{p}\right), \mathrm{GL}_{2}\left(\overline{\mathbf{Q}}_{p}\right)$, $\mathrm{GL}_{1}\left(\overline{\mathbf{Q}}_{p}\right)$, respectively) and take $G=\mathrm{GSp}_{4}$. Here, if the sign is right, the aim would be to find $G_{\mathbf{Q}}$-representations of the shape given by the

\footnotetext{
${ }^{60}$ The notion of CAP automorphic representation is due to Ilya Piatetski-Shapiro (cf. 45]). CAP is an acronym, meaning cuspidal associated to parabolics. These are, indeed, cuspidal automorphic representations closely related to automorphic representations that are induced from a parabolic subgroup. Such cuspidal automorphic representations do not exist on $\mathrm{GL}_{n}$, but for reductive groups for which they do exist, they may sometimes be used in place of Eisenstein series in the context we are discussing. For a reader-friendly brief discussion of this notion, see the introduction to [24].

${ }^{61}$ See the discussion in Section 1 of 3 related to [17.
} 
following picture of this $(1+2+1) \times(1+2+1)$-matrix

$$
\left(\begin{array}{ccc}
\chi & 0 & * \\
* & \rho & * \\
* & 0 & \chi^{*}
\end{array}\right)
$$

whose most "usable" pieces are the submatrices

$$
\left(\begin{array}{ll}
\chi & 0 \\
* & \rho
\end{array}\right)
$$

and

$$
\left(\begin{array}{cc}
\rho & * \\
0 & \chi^{*}
\end{array}\right)
$$

which provide the sought-for extension(s) of Galois representations (these two being equivalent under duality).

This is the format of the article 64, where $\rho$ is taken to be a $G_{\mathbf{Q}^{-}}$ representation attached to a newform of (even) weight $\geq 2$ for $\Gamma_{0}(N)$ (some $N$ ), where the functional equation for the $L$-function would predict that the Selmer group associated to $\rho$ is of odd rank. In this case Skinner and Urban prove that the rank is, at least, positive. As in Bellaïche's thesis, the Eisenstein series (of 6 of Ribet's proof) is replaced by a CAP form. Here, as above, nonordinary p-adic deformations are used to obtain the desired element in the Selmer group.

(4) A variant of (3) above is to keep to the same $(1+d+1) \times(1+d+1)$-matrix picture (for various values of $d$ ) but using a unitary group of rank $d+2$ rather than a (general) symplectic group. Here one would work with initial representations $\chi, \rho, \chi^{*}$ in that order, with $\chi$ some appropriate character and $\rho$ a Galois representation associated with an automorphic form for a unitary group of rank $d$. Chenevier's thesis adopts such a format, and, taking $d=1, E$ a quadratic imaginary field and a unitary group of rank three, the joint work of Bellaïche and Chenevier [5] employs this to find elements in the Selmer groups of certain algebraic Hecke characters over $E$ when the functional equation sign would predict that such elements should exist. Here a nontempered $\pi_{o}$ is used. The forthcoming volume of Bellaïche and Chenevier [6] deals, as well, with examples where $d>1$ in a similar way, achieving interesting results.

(5) Articles by Chris Skinner and Eric Urban (see 62 and 66]) establish the ( $p$-adic) main conjecture for many elliptic curves (defined over $\mathbf{Q}$ ). Here the reductive group in question is $G=\mathrm{GU}(2,2)$, i.e., the general unitary group of signature $(2,2)$ over a quadratic imaginary field $\mathcal{K} / \mathbf{Q}$. The parabolic $P$ is a maximal parabolic subgroup of $G$ fixing an isotropic line; its Levi component is $H=\mathrm{GU}(1,1) \times \operatorname{Res}_{\mathcal{K} / Q} \mathbf{G}_{m}$. The " $\pi_{o}$ " is an Eisenstein series induced from the base change to $\mathcal{K}$ of a cuspform on $\mathrm{GL}_{2}$ over $\mathbf{Q}$ times a Hecke character on $\mathcal{K}$. The $p$-adically varying family of Galois representations " $\rho_{t}$ " is a three-variable family corresponding to a "Hida family" times a two-dimensional space of $p$-adic (degree one) Galois characters over $\mathcal{K}$.

In [65], Skinner and Urban deal with the case where one has a double zero for $L(\rho, s)$ at $s=0$ (when it is the center of the functional equation) to construct two linearly independent extensions of $\mathbf{Q}_{p}(-1)$ by $\rho$ with appropriate Selmer conditions. See, specifically, loc. cit., Theorem B on 
page 475. The strategy is to seek generically irreducible deformations of a representation which has (using the terminology of the beginning of this section) five irreducible constituents $\rho^{(1)}=\rho ; \rho^{(2)}$ and $\rho^{(3)}$ trivial; $\rho^{(4)}$ and $\rho^{(5)}$ given by $\mathbf{Q}_{p}(-1)$.

\section{Appendix I:}

The Preferred Residual REPRESEntation mod $p=691$ of, E.G., $\Delta$

We will be dealing exclusively with the prime $p=691$ in this appendix; for ease of reading though, let $p$ denote the prime 691 below. Let $\Lambda:=\mathbf{Z}_{p}[[\Gamma]]$, where, as usual, $\Gamma \subset \mathbf{Z}_{p}^{*}$ is the group of 1-units. Let $\mathbf{T}$ be the finite flat Hida-Hecke $\Lambda$-algebra (cf. [27, 28, 29] ) that acts naturally on $S_{k}\left(\Gamma_{1}(p) ; \omega^{k-12} ; \mathbf{Z}_{p}\right)^{o}$ for all $k \geq 2$, and acts faithfully on the direct sum

$$
\bigoplus_{k \geq 2} S_{k}\left(\Gamma_{1}(p) ; \omega^{k-12} ; \mathbf{Z}_{p}\right)^{o}
$$

where the superscript $o$ means the $p$-ordinary projection. The classical Hecke operators contained in $\mathbf{T}$ include $T_{\ell}$ for all primes $\ell \neq p$ and the Atkin-Lehner operator $U_{p}$. Let

$$
s_{k}: \Lambda \rightarrow \mathbf{Z}_{p}
$$

be specialization to weight $k$, and denote the $\Lambda$-module by $\mathbf{Z}_{p}$, where the $\Lambda$-action is given via $s_{k}$ by the symbol $\mathbf{Z}_{p}\langle k\rangle$.

Lemma 5. (1) The natural ring homomorphism

$$
\Lambda \rightarrow \mathbf{T}
$$

is an isomorphism.

(2) For every $k$ the natural homomorphism of $\Lambda$-modules

$$
\mathbf{Z}_{p}\langle k\rangle \longrightarrow \mathbf{T} \otimes_{\Lambda} \mathbf{Z}_{p}\langle k\rangle
$$

is an isomorphism.

Proof. Clearly (1) implies (2). Since $\mathbf{T}$ is a finite flat $\Lambda$-algebra, (1) follows if (2) holds for some value of $k$. One computes that the $\mathbf{Z}_{p}$-module $S_{12}\left(\Gamma_{1}(p) ; \omega^{0} ; \mathbf{Z}_{p}\right)^{o}$ is generated by the ordinary eigenform $\Delta(z)-p^{11} \Delta(p z)$. Since $\mathbf{T} \otimes_{\Lambda} \mathbf{Z}_{p}\langle 12\rangle$ acts faithfully on $S_{12}\left(\Gamma_{1}(p) ; \omega^{0} ; \mathbf{Z}_{p}\right)^{\circ}$ as follows from Hida's theory (cf. Thm. 22 of [30]), we see that (2) holds for $k=12$, which is enough to prove the lemma. Independently, computations of Stein and Citro establish (2) for $k=2$.

Hida's theory (cf. loc. cit.) gives a degree two pseudo-representation over $\mathbf{T}=\Lambda$ which associates to the Frobenius element at a prime $\ell \neq 691$ the Hecke operator $T_{\ell} \in \mathbf{T}$. When specialized to weight two this (pseudo-)representation yields the Galois representation over $\mathcal{F}_{v}=\mathbf{Q}_{691}$ associated to $A_{f}$, the abelian variety discussed in Section 6. We can realize the entire pseudo-representation over $\mathbf{T}$ by an honest Galois representation, $\rho_{\mathbf{T}}: G_{\mathbf{Q}} \rightarrow \mathrm{GL}_{2}(\mathbf{T})=\mathrm{GL}_{2}(\Lambda)$ using Proposition 1.6 .1 of [6], since the pseudo-representation is residually multiplicity free and $\mathbf{T}$ is a factorial local ring.

The relevant Leopoldt-Kubota $L$-function $\mathcal{L} \in \Lambda$ is not divisible by $p$ (which is a general phenomenon thanks to the " $\mu=0$ " result of [23]) and has a single zero of multiplicity 1 (as had been computed long ago). Equivalently, $\Lambda / \mathcal{L} \Lambda \simeq \mathbf{Z}_{p}$. 
The Eisenstein ideal $I \subset \mathbf{T}$ is the closed ideal in $\mathbf{T}$ generated by the elements $T_{\ell}-1-\langle\ell\rangle \ell^{-1}$ (for $\ell \neq p$ ) and the element $U_{p}-1$. By [22] (or [41]) we see that under the natural isomorphism $\Lambda \rightarrow \mathbf{T}$, the element $\mathcal{L}$ is sent to a generator of the Eisenstein ideal $I$. We can, if we wish, view $\mathcal{L}$ as a parameter in $\Lambda=\mathbf{T}$ in the sense that we have natural isomorphisms

$$
\Lambda=\mathbf{T}=\mathbf{Z}_{p}[[\mathcal{L}]]
$$

Reducing $\rho_{\mathbf{T}}$ modulo $\mathcal{L}$ one gets a representation

$$
\rho_{\mathbf{T}, \bmod \mathcal{L}}: G_{\mathbf{Q}} \rightarrow \mathrm{GL}_{2}\left(\mathbf{Z}_{p}\right) \subset \mathrm{GL}_{2}\left(\mathbf{Q}_{p}\right),
$$

and we have (using the above two paragraphs) that the character of this dimension two $G_{\mathbf{Q}}$-representation over $\mathbf{Q}_{p}$ is the sum of two one-dimensional characters: the trivial character and the determinant $\operatorname{det}\left(\rho_{\mathbf{T}, \bmod } \mathcal{L}\right)$. It follows that $\rho_{\mathbf{T}, \bmod \mathcal{L}}$ is reducible. Choose a basis $\{\bar{x}, \bar{y}\}$ for a $\rho_{\mathbf{T}, \bmod } \mathcal{L}^{\text {-stable lattice }}$

$$
\bar{M} \subset \mathbf{Z}_{p} \oplus \mathbf{Z}_{p}=\Lambda /(\mathcal{L}) \oplus \Lambda /(\mathcal{L})
$$

such that the action of $G_{\mathbf{Q}}$ is upper-triangular; lift this basis to obtain a basis $\{x, y\}$ for the underlying $\Lambda$-module of the representation $G_{\mathbf{Q}} \rightarrow \mathrm{GL}_{2}(\Lambda)$ (which we write, in terms of this basis, as $\Lambda \oplus \Lambda$ ) so that the action of $G_{\mathbf{Q}}$ is upper-triangular modulo $\mathcal{L} \Lambda$.

In particular, the "sublattice" $M^{\prime}:=\mathcal{L} \Lambda \oplus \Lambda \subset M:=\Lambda \oplus \Lambda$ is $G_{\mathbf{Q} \text {-stable so }}$ that we have the option of taking either $M$ or $M^{\prime}$ as our basic lattice in terms of which we will write the representation $\rho_{\mathbf{T}}$. This boils down to considering either the initial representation $\rho_{\mathbf{T}}$ or its conjugate:

$$
\left(\begin{array}{cc}
\mathcal{L} & 0 \\
0 & 1
\end{array}\right) \cdot \rho_{\mathbf{T}} \cdot\left(\begin{array}{cc}
\mathcal{L}^{-1} & 0 \\
0 & 1
\end{array}\right) .
$$

By the residual representations attached to the $\Lambda$-lattices $M$ and $M^{\prime}$ we mean

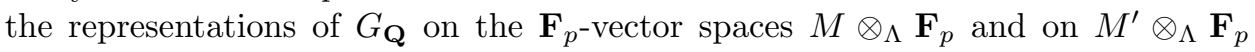
obtained by reduction modulo the maximal ideal of $\Lambda$.

- Reduction to weight twelve. Reducing the representation $\rho_{\mathbf{T}}: G_{\mathbf{Q}} \rightarrow$ $\mathrm{GL}_{2}(\mathbf{T})$ to weight twelve, i.e., composing with the homomorphism $\mathrm{GL}_{2}(\mathbf{T})$ $\rightarrow \mathrm{GL}_{2}\left(\mathbf{Z}_{p}\right)$ induced from the ring homomorphism $s_{12}: \Lambda \rightarrow \mathbf{Z}_{p}$, we get from either lattice $M$ or $M^{\prime}$ above, two $G_{\mathbf{Q}}$-stable $\mathbf{Z}_{p}$-lattices for the representation $\rho_{\Delta, 691}$. Call them $M_{12}$ and $M_{12}^{\prime}$. The residual representations attached to the $\Lambda$-lattices $M$ and $M^{\prime}$ are naturally isomorphic to residual representations attached to the $\mathbf{Z}_{p}$-lattices $M_{12}$ and $M_{12}^{\prime}$, respectively. By Proposition 1 the only residual representations for $G_{\mathbf{Q}^{-}}$-stable lattices of $\rho_{\Delta, 691}$ are (reducible and) indecomposable, and in their Jordan-Hölder decompositions the one-dimensional characters occur in a different order. By Corollary 6, one of these when restricted to the inertia group at $p$ is semisimple, and the other is not. It follows that the residual representations attached to $M_{12}$ and $M_{12}^{\prime}$, and consequently also the residual representations attached to the $\Lambda$-lattices $M$ and $M^{\prime}$, are indecomposable representations $G_{\mathbf{Q}} \rightarrow \mathrm{GL}_{2}\left(\mathbf{F}_{p}\right)$ with characters $\mathbf{1}$ and $\omega^{11}$ occurring in the two different possible orderings; one of these when restricted to the inertia group at $p$ is semisimple, and the other is not.

- Reduction to arbitrary weight. Reducing the representation $\rho_{\mathbf{T}}: G_{\mathbf{Q}} \rightarrow$ $\mathrm{GL}_{2}(\mathbf{T})$ to any weight $w \geq 2$, for reasons similar to the above we get 
that the two indecomposable residual representations attached to lattices for $\rho_{w, 691}$ are independent of $w \geq 2$ and are isomorphic to the residual representations attached to $\rho_{\mathbf{T}, M}$ and $\rho_{\mathbf{T}, M^{\prime}}$.

Definition 3. Call the indecomposable residual representation for which the character 1 occurs as a quotient representation, rather than a sub-representation of the residual representation, the preferred indecomposable residual representation.

In particular, the preferred residual representation of $\rho_{w, 691}$ is independent of the weight $w$.

- Reduction to weight two. For reasons analogous to those occurring in the first bullet in Section [18, using properties of the abelian variety $A_{f}$ (of Section [6) one obtains (as in [4]) a proof, slightly different than the one above, that the preferred residual representation attached to $\rho_{2,691}$ has the property that its inertial action is semisimple 62

- Anomalous representations. It may deserve mention that the only anomalous representation in this Hida family is the Iwasawa representation (i.e., the representation into $\mathrm{GL}_{2}\left(\mathbf{Z}_{691}\right)$ obtained by reducing modulo $\left.\mathcal{L}\right)$. For by [41] or 22], we have that $\mathcal{L}$ divides $U_{691}-1$ in $\Lambda=\mathbf{T}$. Write

$$
U_{691}-1=\mathcal{N} \cdot \mathcal{L} \in \Lambda,
$$

for $\mathcal{N} \in \Lambda$. Let the covering symbol tilde denote the image in $\mathbf{Z}_{p}$ of elements in $\Lambda$ under the ring homomorphism $s_{12}: \Lambda \rightarrow \mathbf{Z}_{p}$.

The image (under $s_{12}$ ) of the Hecke operator $U_{691}$ is congruent to $\tau(691)$ $\bmod 691^{11}$. A computation using SAGE 63 tells us that $\tau(691) \equiv 374523=$ $1+542 \cdot 691$ modulo $691^{2}$, so $\tilde{U}_{691}-1$ is divisible by 691 but not by $691^{2}$. Since $\tilde{\mathcal{L}} \equiv 0 \bmod 691$ it follows that $\tilde{\mathcal{N}}$ is a unit in $\mathbf{Z}_{p}$, and therefore that $\mathcal{N}$ is a unit in $\Lambda$.

So we have an equality of ideals $\left(U_{691}-1\right)=(\mathcal{L})$ in $\Lambda$. But the ideal $\left(U_{691}-1\right)$ is the defining ideal in $\mathbf{T}$ of the locus of anomalous quotient representations (derived from $\rho_{\mathbf{T}}$ ), proving our assertion.

\section{ApPEndiX II: \\ COMPUTATIONS FOR MORE IRREGULAR PAIRS}

Appendix I above has been a close study of the irregular pair $(p, 2 k)=(691,12)$ and gives us information about the nature of the package of newforms given in station $\mathbf{5}$ in the Herbrand-Ribet theorem (Theorem 1 of Section 6) related to $(p, 2 k)=(691,12)$. The modular form in this package of newforms that is of level 1 and of lowest weight (i.e., weight 12) is $\Delta$, and we have discussed this case somewhat. We have also shown that there is a unique newform in our package in each weight $\geq 2$ that is congruent modulo 691 to the Eisenstein series of that weight. Appendix I concommitantly tells us something about the corresponding

\footnotetext{
${ }^{62}$ Note that the representation $\rho_{2,691}$ is obtained by the action of Galois on cohomology and therefore is dual to the associated representation that occurs within $V_{691}\left(A_{f}\right)$, the tensor product over $\mathbf{Q}_{691}$ of the 691-adic Tate module obtained from $691^{n}$-torsion points of the abelian variety $A_{f}$.

${ }^{63}$ Computations and a theoretical discussion related to this can be found in 69, where it is shown, among other things, that the image of Galois under $\rho_{12,691}$ in $\mathrm{GL}_{2}\left(\mathbf{Z}_{691}\right)$ is as large as it can be, given that the image of its associated residual representation lies in a Borel subgroup of $\mathrm{GL}_{2}\left(\mathbf{F}_{691}\right)$.
} 
Hida family (consisting of 691-ordinary 691-adic modular eigenforms of tame level 1 congruent to the corresponding Eisenstein series "mod 691").

What happens for other irregular pairs:64

For many conceptual questions in the arithmetic of cyclotomic fields, it is probably not a good idea to use extensive computation as a trustworthy guide to a conjectured answer to a general question. Consider, for example, Vandiver's conjecture, which is verified for primes $<163,000,000$ ([10]). Here is what Joe Buhler and David Harvey say about that computation:

We find that the expected number of counterexamples up to 12 million is about 0.674 , and that another 0.074 counterexamples were expected between 12 million and 163 million (though of course we now know that there are none in either case). Many people believe that Vandiver's conjecture is true; it also seems reasonable to believe that the conjecture is false but that the first counterexample is so astronomically large that it may never be known.

Nevertheless, some computations of William Stein for irregular pairs $(p, 2 k)$ with $p<10^{7}$ and $2 k<8000$, give rather striking information about the nature of the package of newforms given in station $\mathbf{5}$ in the Herbrand-Ribet theorem (Theorem 1 of Section 6). Here, again, the modular form in this package of newforms that is of level 1 and of lowest weight is of weight $2 k$.

Specifically, Stein shows that for irregular pairs $(p, 2 k)$ with $p<10^{7}$ and $2 k<$ 8000 , with one exception there is a unique eigenform of level 1 in weight $w=2 k$ for which there is a prime ideal $P$ in the ring $O$ generated by its Fourier coefficients such that $O / P=\mathbf{F}_{p}$ and its eigenvalue for the Hecke operator $T_{2}$ is congruent $\bmod P$ to the eigenvalue for the Hecke operator $T_{2}$ acting on the Eisenstein series of weight $k$, taken mod $p$. In particular, in these examples, the eigenvalue of the Hecke operator $T_{2} \bmod p$ alone is enough to "cut out" the eigenform of weight $2 k$ in the package of newforms given in station $\mathbf{5}$.

The exceptional irregular pair is $(p, 2 k)=(547,486)$. For this irregular pair, there is a conjugate pair of newforms of weight 486 with the required Eisenstein congruence condition "mod 547", each with Fourier coefficients generating the quadratic extension $\mathbf{Q}_{p}(\sqrt{-} p)$ (and for this case too, the Hecke operator $T_{2}$ also cuts out the conjugate pair of newforms of weight 486 out of the space of all newforms of level 1 of that weight).

\footnotetext{
${ }^{64}$ E.g., here are the first few irregular pairs $(p, 2 k)$ ordered by increasing weight $w=2 k$ :

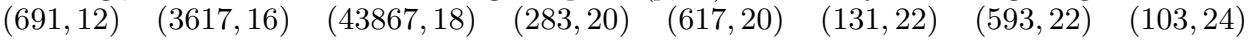

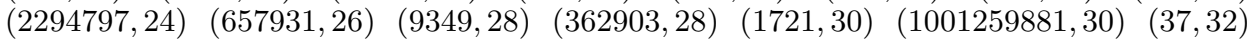
$(683,32) \quad(305065927,32) \quad(151628697551,34) \quad(26315271553053477373,36)$

65 There is a companion pair of side questions to this phenomenon:
}

(1) Are there nonordinary cuspidal eigenforms of level 1 and weight $2 k$ with Fourier coefficients in $\mathcal{O}$, the ring of integers in a number field, and is there a prime $P \subset \mathcal{O}$ of residual characteristic $p$ and degree one, such that the semisimplification of the associated residual representation mod $P$ is the direct sum of the characters 1 and $\omega^{2 k-1}$ ?

(2) If there are such eigenforms, can one still obtain a construction of the requisite unramified abelian extension of $\mathbf{Q}\left(e^{2 \pi i / p}\right)$ (using, perhaps, Fontaine's theory) in a manner analogous to the method that works in the ordinary case?

Regarding the first question, Stein's computations tell us that there is no such (nonordinary) eigenform if $p<10^{7}$ and $k<8000$. The second question is the issue dealt with in [34] (this was also discussed by C. Dalawat, in lectures given at Gauhati). 
One can show, following arguments as in Appendix I, that for all cases $p<10^{7}$ and $2 k<8000$, except for $(p, 2 k)=(547,486)$, the corresponding Hida Hecke algebra $\mathbf{T}$ is isomorphic to the Iwasawa algebra $\Lambda$.

When $(p, 2 k)=(547,486)$ the corresponding Hecke algebra, $\mathbf{T}$, is finite flat of rank two over $\Lambda$. What is its discriminant ideal? Is $\mathbf{T}$ an integral domain? Is its spectrum geometrically unibranch? (That is, is $\overline{\mathbf{Q}}_{p} \otimes \mathbf{z}_{p} \mathbf{T}$ an integral domain?)

To answer this, one might be led to computing (special values of) $p$-adic $L$ functions associated to the symmetric squares of the relevant classical modular eigenforms.

More generally, to get closer to the numerical phenomena surveyed in this article, it seems to me that a good deal of further numerical experimentation (in various directions) is warranted. For example, in view of the immense amount of computation that has been focused on classical irregular pairs, and on the classical $p$-adic $L$-functions $L_{p}\left(s, \omega^{2 k}\right)$, it might be a good idea to extend and round out this database to include, as far as is practical, information about the analogous problems posed by $p$-adic $L$-functions $L_{p}\left(s, \alpha \cdot \omega^{2 k}\right)$, where $\alpha$ is a finite (Dirichlet) character of prime-to- $p$ conductor.

\section{About the AUTHOR}

Barry Mazur is the Gerhard Gade University Professor at Harvard University.

\section{REFERENCES}

[1] E. Artin, J. Tate, Class Field Theory, Addison-Wesley, 1990. MR.1043169 (91b:11129)

[2] J. Bellaïche, Relèvement des formes modulaires de Picard, J. London Math. Soc. (2), no. 1, 74, 13-25 (2006). MR.2254549 (2007f:11050)

[3] J. Bellaïche, Congruences endoscopiques et représentations galoisiennes, Thesis, Université Paris-Sud, 2002.

[4] J. Bellaïche, A propos d'un lemme de Ribet, Rend. Sem. Mat. Univ. Padova 109, 45-62 (2003). MR1997986 (2004h:20064)

[5] J. Bellä̈che, G. Chenevier, Formes non tempérées pour U(3) et conjectures de BlochKato, Annales scientifiques de l'Ecole Norm. Sup. 37 no. 4, 611-662 (2004). MR2097894 (2005m:11096)

[6] J. Bellaïche, G. Chenevier, Families of Galois representations and Selmer groups, Astérisque 324 (2009). MR2656025

[7] J. Bernoulli, The Art of Conjecturing, together with Letter to a Friend on Sets in Court Tennis; English Translation and Commentary by Edith Dudley Sylla, The Johns Hopkins Press, 2006. MR.2195221 (2006j:01006)

[8] R. Brauer, C. Nesbitt, On the modular characters of groups, Ann. of Math. (2) 42, 556-590 (1941). MR.0004042 (2:309c)

[9] J. Buhler, R. Crandall, R. Ernval, T. Metsänkylä, M. Amin Shokrollahie, Irregular primes and cyclotomic invariants to 12 million, Journal of Symbolic Computation 31, 89-96 (2001). MR.1806208 (2001m:11220)

[10] J. Buhler, D. Harvey, Irregular primes to 163 million, arXiv:0912.2121v2 [math.NT] (2009).

[11] F. Calegari, B. Mazur, Nearly ordinary Galois deformations over arbitrary number fields, Journal of the Institute of Mathematics of Jussieu, 8, Issue 01, 99-177 (2009). MR2461903 (2009i:11070)

[12] Algebraic Number Theory (Eds. J. W. S. Cassel, A. Fröhlich, Proceedings of an Instructional Conference, London Mathematical Society, 1967. MR911121 (88h:11073)

[13] N. Cebotarev, Bestimmung der Dichtigkeit einer Menge von Primzahlen, welche zu einer gegebenen Substitutionsklasse gehören, Math. Ann. 95, 191-228 (1926). MR1512273

[14] G. Chenevier, Familles $p$-adiques de formes automorphes pour GL $(n)$, Journal für die reine und angewandte Mathematik 570, 143-217 (2004). MR2075765 (2006b:11046) 
[15] G. Chenevier, Une correspondance de Jacquet-Langlands p-adique, Duke Math. Journal 126 no. 1, 161-194 (2005). MR2111512 (2006f:11144)

[16] G. Chenevier, The $p$-adic analytic space of pseudo-characters of a profinite group and pseudorepresentations over arbitrary rings, arXiv:0809.0415v1 [math.NT] Sept. 2 (2008).

[17] L. Clozel, On Ribet's Level-raising Theorem for U(3), American Journal of Math. 122, 12651287 (2000). MR 1797662 (2001k:11087)

[18] C. Curtis, I. Reiner, Representation theory of finite groups and associative algebras, Wiley, 1962. MR1013113 (90g:16001)

[19] P. Deligne, Formes modulaires et représentations $\ell$-adiques, Séminaire Bourbaki, Lect. Notes in Math. 1799 Springer, 139-172 (1971).

[20] P. Deligne, M. Rapoport, Les schémas de modules de courbes elliptiques, Modular functions of one variable, II (Proc. Internat. Summer School, Univ. Antwerp, Antwerp, 1972), pp. 143-316 in Lecture Notes in Math. 349, Springer, 1973. MR0337993 (49:2762)

[21] P. Deligne, J.-P. Serre, Formes modulaires de poids 1. Annales de l'Ecole Norm. Sup. 74, 507-530 (1974). MR0379379 (52:284)

[22] M. Emerton, The Eisenstein ideal in Hida's ordinary Hecke algebra, Internat. Math. Res. Notices 15, 793-802 (1999). MR,1710074 (2000e:11057)

[23] B. Ferrero, L. Washington, The Iwasawa invariant $\mu_{p}$ vanishes for abelian number fields, Ann. of Math. (2) 109, 377-395 (1979). MR.528968(81a:12005)

[24] W.T. Gan, N. Gurevich, CAP representations of $G_{2}$ and the spin $L$-function of PGSp 6 , Israel J. Math. 170, 1-52 (2009). MR2506316 (2010c:22023)

[25] L. R. Graham, J.M. Kantor, Naming Infinity, Belknap Press, 2009. MR 2526973 (2010c:01007)

[26] J. Herbrand, Sur les classes des corps circulaires, J. Math. Pure Appl. (9) II 417-441 (1932).

[27] H. Hida, On $p$-adic Hecke algebras for $\mathrm{GL}_{2}$ over totally real fields, Ann. of Math. (2) 128, 295-384 (1988). MR960949 (89m:11046)

[28] H. Hida, On nearly ordinary Hecke algebras for GL(2) over totally real fields, Advanced Studies in Pure Math. 17, 139-169 (1989). MR.1097614 (92f:11064)

[29] H. Hida, $p$-Adic ordinary Hecke algebras for GL(2), Ann. de l'Institut Fourier 44, 1289-1322 (1994). MR1313784 (95k:11065)

[30] H. Hida, Control Theorems and Applications, Lectures at Tata Institute of Fundamental Research (Version of 2/15/00) [See http://www.math.ucla.edu/ hida/]

[31] H. Hida, Hilbert Modular Forms and Iwasawa Theory, Oxford University Press, 2006. MR2243770 (2007h:11055)

[32] H. Hida, J. Tilouine and E. Urban, Adjoint modular Galois representations and their Selmer groups, Proc. Natl. Acad. Sci. USA 94, 11121-11124 (1997). MR1491970 (98m:11034)

[33] C. Khare, Serre's modularity conjecture: a survey of the level one case, pp. 270-299 in $L$ functions and Galois representations, London Math. Soc. Lecture Note Ser., 320, Cambridge Univ. Press, 2007. MR 2392357 (2009g:11066)

[34] C. Khare, Notes on Ribet's converse to Herbrand, Cyclotomic fields, Bhaskaracharya Pratishthana, Poona, 273-284 (2000). MR1802388 (2002e:11143)

[35] C. Khare, J.-P. Wintenberger, On Serre's conjecture for 2-dimensional mod $p$ representations of $\operatorname{Gal}(\overline{\mathbf{Q}} / \mathbf{Q})$, Ann. of Math. (2) 169 no. 1, 229-253 (2009). MR2480604 (2009m:11077)

[36] C. Khare, J.-P. Wintenberger, Jean-Pierre Serre's modularity conjecture. I; and II. Invent. Math. 178, 485-504 and 505-586 (2009). MR2551763 (2010k:11087); MR2551764 (2010k:11088)

[37] M. Kisin, Overconvergent modular forms and the Fontaine-Mazur conjecture, Invent. Math. 153 (2) 373-454 (2003). MR 1992017 (2004f:11053)

[38] M. Koike, Congruences between cuspforms of weight one and of weight two and a remark on a theorem of Deligne and Serre (Int. Symposium on Algebraic Number Theory, Kyoto, March 1976).

[39] S. Lang, Algebraic Number Theory (Second Edition) Springer, 1993. MR.1282723 (95f:11085)

[40] B.Mazur, Deforming Galois representations, pp. 385-437 in Galois groups over Q, Math. Sci. Res. Inst. Publ., 16, Springer, 1989. MR.1012172 (90k:11057)

[41] B. Mazur, A. Wiles, Analogies between function fields and number fields, Amer. J. Math. 105, 507-521 (1983). MR701567 (84g:12003)

[42] B. Mazur, A. Wiles, Class fields of abelian extensions of Q, Invent. Math. 76 no.2, 179-330 (1984). MR742853 (85m:11069) 
[43] B. Mazur, A. Wiles, $p$-adic analytic families of Galois representations, Compositio Math. 59 231-264 (1986). MR860140 (88e:11048)

[44] I. Piatetski-Shapiro, Two Conjectures on $L$-functions, pp. 519-522 in Wolf Prize in Mathematics Vol 2. (Eds. S.S. Chern and F. Hirzebruch) World Scientific, 2000.

[45] I. Piatetski-Shapiro, On the Saito-Kurokawa lifting, Invent. Math., 71(2) 309-338 (1983). MR689647 (84e:10038)

[46] F. Pollaczek, Uber die irregulären Kreiskörper der $\ell$-ten und $\ell^{2}$-ten Einheitswurzeln, Math. Zeit. 21, 1-38 (1924). MR1544682

[47] Srinivasa Ramanujan, On certain arithmetical functions, Trans. Cambridge Philos. Soc. 22 (9) 159-184 (1916).

[48] M. Raynaud, Schémas en groupes de type $(p, p, \ldots, p)$, Bull. Soc. Math. France. 102, 241-280 (1974). MR0419467 (54:7488)

[49] K. Ribet, A modular construction of unramified p-extensions of $\mathbf{Q}\left(\mu_{p}\right)$, Inventiones Math. 34, 151-162 (1976). MR0419403 (54:7424)

[50] K. Ribet, Galois representations attached to eigenforms with nebentypus, pp. 18-52 in Modular Functions of one Variable V, Lecture Notes in Mathematics, 601, Springer, 1977. MR0453647 (56:11907)

[51] K. Ribet and W. Stein, Lectures on Serres' conjectures, pp. 143-232 in Arithmetic algebraic geometry (Park City, UT, 1999), IAS/Park City Math. Ser. 9, Amer. Math. Soc., Providence, RI, 2001. MR1860042(2002h:11047)

[52] D. Rohrlich, Modular Curves, Hecke Correspondences, and L-functions, pp.41-99 in Modular Forms and Fermat's Last Theorem, Springer, 1997. MR.1638476

[53] J.-P. Serre, Zeta and L-functions, Arithmetical Algebraic Geometry, Harper and Row, New York, 1965. MR0194396 (33:2606)

[54] J.-P. Serre, Abelian $\ell$-adic Representations and Elliptic Curves, W.A. Benjamin, Inc., 1968. MR0263823 (41:8422)

[55] J.-P. Serre, Une interprétation des congruences relatives à la fonction $\tau$ de Ramanujan, pp. 498-511 in Jean-Pierre Serre, Oeuvres, Collected Papers, Volume II (1960-1971) Springer, 1986. MR0926690 (89h:01109b)

[56] J.-P. Serre, Modular Forms of weight one and Galois Representations, pp. 292-367 in JeanPierre Serre, Oeuvres, Collected Papers, Volume III (1972-1984) Springer, 1986. MR0926691 (89h:01109c)

[57] J.-P. Serre, Représentations $\ell$-adiques, pp. 384-401 in Jean-Pierre Serre, Oeuvres, Collected Papers, Volume III (1972-1984) Springer, 1986. MR0926691(89h:01109c)

[58] J.-P. Serre, Trees (transl. J. Stillwell) Springer Monographs in Mathematics (2003). MR.1954121 (2003m:20032)

[59] J.-P. Serre, Sur les représentations modulaires de degré 2 de $\operatorname{Gal}(\overline{\mathbf{Q}} / \mathbf{Q})$, Duke Math. J. 54, 179-230 (1987). MR885783 (88g:11022)

[60] G. Shimura, On the factors of the Jacobian variety of a modular function field, J. Math. Soc. Japan 25, 523-544 (1973). MR0318162 (47:6709)

[61] G. Shimura, Introduction to Arithmetic Theory of Automorphic Functions, Publ. Math. Soc. Japan, 11, Tokyo-Princeton (1971). MR0314766 (47:3318)

[62] C. Skinner, Elliptic Curves and Main Conjectures, Kuwait Foundation Lecture 49, May 24, 2005. http://www.dpmms.cam.ac.uk/Seminars/Kuwait/abstracts/L49.pdf

[63] C. Skinner, A. Wiles, Residually reducible representations and modular forms, Publications Mathématiques de l'IHÉS 89, 6-126 (1999). MR1793414 (2002b:11072)

[64] C. Skinner, E. Urban, Sur les déformations $p$-adiques de certaines représentations automorphes. J. Inst. Math. Jussieu 5 no. 4. 629-698 (2006). MR2261226(2008a:11072)

[65] C. Skinner, E. Urban, Vanishing of $L$-functions and ranks of Selmer groups, pp. 473-500 in Proceedings of the International Congress of Mathematicians. Vol. II, Eur. Math. Soc., Zurich (2006); See also: http://www.math.columbia.edu/ urban/EURP08.html. MR2275606 (2008a:11063)

[66] C. Skinner, E. Urban, The main conjecture for $\mathrm{GL}_{2}$; See: http://www.math.columbia.edu/ $\sim$ urban/EURP08.html

[67] W. Stein, An introduction to computing modular forms using modular symbols, pp. 642-652 in Algorithmic Number Theory, MSRI Publications 44 (2008). MR2467560 (2009k:11085) 
[68] H. P. F. Swinnerton-Dyer, On $\ell$-adic representations and congruences for coefficients of modular forms, I: pp. 1-55 in Modular functions of one variable, III, Lecture Notes in Mathematics, 350, Springer, 1973. MR0406931 (53:10717a)

[69] H. P. F. Swinnerton-Dyer, On $\ell$-adic representations and congruences for coefficients of modular forms, II: pp. 63-90 in Modular functions of one variable, $V$, Lecture Notes in Mathematics, 601, Springer, 1977. MR0498392 (58:16520)

[70] E. Urban, On residually reducible representations on local rings, J. Algebra 212 no. 2, 738-742 (1999). MR 1676863 (2000a:16020)

[71] E. Urban, Selmer groups and the Eisenstein-Klingen ideal, Duke Math. J. 106 no. 3, 485-525 (2001). MR 1813234(2002b:11073)

[72] E. Urban, Groupes de Selmer et Fonctions $L$-adiques pour les représentations modulaires adjointes, See: http://www.math.columbia.edu/ urban/EURP08.html

[73] H. Vandiver, Fermat's Last Theorem: Its history and the nature of the known results concerning it, Amer. Math. Monthly 53, 555-578 (1946); 60, 164-167 (1953).

[74] L. Washington, Introduction to Cyclotomic Fields, Springer, 1982. MR718674 (85g:11001)

[75] A. Wiles, On ordinary $\lambda$-adic representations associated to modular forms, Invent. Math. 94, 529-573 (1988). MR 969243 (89j:11051)

[76] A. Wiles, The Iwasawa conjecture for totally real fields, Ann. of Math. (2) 131, 493-540 (1990). MR.1053488 (91i:11163)

Department of Mathematics, Harvard University, Cambridge, Massachusetts 